NASA Contractor Report 4310

\title{
Design and Implementation of Robust Decentralized Control Laws for the ACES Structure at Marshall Space Flight Center
}

Emmanuel G. Collins, Jr., Douglas J. Phillips, and David C. Hyland

Harris Corporation

Government Aerospace Systems Division

Melbourne, Florida

Prepared for

Langley Research Center

under Contract NAS1-18872

\section{Nons}

National Aeronautics and

Space Administration

Office of Management

Scientific and Technical Information Division 



\begin{abstract}
Many large space system concepts will require active vibration control to satisfy critical performance requirements such as line-of-sight accuracy. In order for these concepts to become operational it is imperative that the benefits of active vibration control be practically demonstrated in groundbased experiments. This report describes an experiment conducted by Harris as part of the NASA CSI Guest Investigator Program. The results of this experiment successfully demonstrate active vibration control for a flexible structure. The testbed is the ACES structure at NASA Marshall Space Flight Center. The ACES structure is dynamically traceable to future space systems and especially allows the study of line-of-sight control issues.
\end{abstract}



Table of Contents

1.0 Introduction . . . . . . . . . . . . . . . . . . . . . . . . 1-1

2.0 Description of the ACES Structure . . . . . . . . . . . . . . . . . . . . 2-1

3.0 Modeling Procedure for the ACES Structure . . . . . . . . . . . . . . . 3-1

3.1 Choosing the Modeling Procedure . . . . . . . . . . . . . . . . . . . . . . 3-1

3.2 Model Development Using the Eigensystem Realization Algorithm . . . . . . . 3-3

4.0 Control Design for the ACES Structure . . . . . . . . . . . . . . . . . . 4-1

4.1 Design Process for the Detector to IMC Loops . . . . . . . . . . . . . . . . 4-1

4.2 Design Process for the Base Gyro to AGS Gimbal Loops . . . . . . . . . . . . 4-2

4.3 Design Process for the LMED Force to Accelerometer Loops . . . . . . . . . . 4-3

5.0 Performance of the Integrated Controller . . . . . . . . . . . . . . . . . $5-1$

5.1 Response Due to Pulse Disturbances . . . . . . . . . . . . . . . . . . . . 5-1

5.2 Response Due to to an RCS Disturbances . . . . . . . . . . . . . . . . . . 5-2

5.3 Response Due to a Crew Disturbances . . . . . . . . . . . . . . . . . . . . 5-2

5.4 Some Final Remarks on the Implementation Results . . . . . . . . . . . . . 5-2

6.0 Closing Remarks and Conclusions . . . . . . . . . . . . . . . . . . . . . 6-1

7.0 References . . . . . . . . . . . . . . . . . . . . . . . . . 7-1

8.0 Appendix . . . . . . . . . . . . . . . . . . . . . . . . . . . . . . . . . 8-1 



\section{List of Tables}

5.1.1 Det-X Response Improvement to a BET-X Pulse . . . . . . . . . . . . . . . . 5-3 As the Controllers Were Combined

5.1.2 Det-Y Response Improvement to a BET-Y Pulse . . . . . . . . . . . . . . . . 5-4 As the Controllers Were Combined

\section{List of Figures}

1.1 The Maximum Entropy/Optimal Projection . . . . . . . . . . . . . . . . . . 1-3 Design Equations

2.1 The ACES Test Article . . . . . . . . . . . . . . . . . . . . . . . . . . $2-5$

3.1.1 AGS-X to BGYRO-X: Magnitude Comparison of . . . . . . . . . . . . . . . . 3-4 FE Model Bode Plot vs. FRF

3.1.2 AGS-Y to BGYRO-Y: Magnitude Comparison of . . . . . . . . . . . . . . . . 3-5 FE Model Bode Plot vs. FRF

3.1.3 AGS-X to BGYRO-X: Phase Comparison of . . . . . . . . . . . . . . . . . . 3-6 FE Model Bode Plot vs. FRF

3.1.4 IMC-X to DET-Y: Magnitude Comparison of . . . . . . . . . . . . . . . . . 3-7 FE Model Bode Plot vs. FRF

3.1.5 IMC-Y to DET-X: Magnitude Comparison of . . . . . . . . . . . . . . . . . 3-8 FE Model Bode Plot vs. FRF

3.1.6 Pattern of Strong and Weak Interactions . . . . . . . . . . . . . . . . . . . 3-9 in the System Dynamics

3.2.1 Iterative Procedure for System Identification . . . . . . . . . . . . . . . . . . 3-10

3.2.2 Final Steps in Model Development for . . . . . . . . . . . . . . . . . . . . . 3-11 AGS-X to BGYRO-X and AGS-Y to BGYRO-Y Loops

vii 
3.2.3 Final Steps in Model Development for . . . . . . . . . . . . . . . . . . . . 3-12 IMC-X to DET-Y and IMC-Y to DET-X Loops

3.2.4 AGS-X to BGYRO-X: Magnitude Comparison of . . . . . . . . . . . . . . . . 3-13 ERA Model Bode Plot vs. FRF

3.2.5 AGS-Y to BGYRO-Y: Magnitude Comparison of . . . . . . . . . . . . . . . . 3-14 ERA Model Bode Plot vs. FRF

3.2.6 AGS-X to BGYRO-X: Phase Comparison of . . . . . . . . . . . . . . . . . . 3-15 ERA Model Bode Plot vs. FRF

3.2.7 IMC-X to DET-Y: Magnitude Comparison of . . . . . . . . . . . . . . . . . . 3-16 ERA Model Bode Plot vs. FRF

3.2.8 IMC-Y to DET-X: Magnitude Comparison of . . . . . . . . . . . . . . . . . . 3-17 ERA Model Bode Plot vs. FRF

4.1.1 Disturbance Tracking Problem for the IMC . . . . . . . . . . . . . . . . . . 4-5 to DET Loops

4.1.2 DET-X Response to a BET-X Pulse: . . . . . . . . . . . . . . . . . . . . . 4-6 Open Loop vs. Closed Loop with the IMC to DET Controller

4.1.3 DET-Y Response to a BET-Y Pulse: . . . . . . . . . . . . . . . . . . . . . 4-7 Open Loop vs. Closed Loop with the IMC to DET Controller

4.2.1 Influence of Maximum Entropy Design on Phase in the Performance . . . . . . . 4-8 Region for the AGS to BGYRO Loops

4.2.2 Influence of Maximum Entropy Design on Magnitude in the Performance . . . . . 4-9 Region for the AGS to BGYRO Loops

4.2.3 Maximum Entropy Design Robustified the . . . . . . . . . . . . . . . . . . . 4-10 Notches for the High Frequency Modes

4.2.4 DET-X Response to a BET-X Pulse: . . . . . . . . . . . . . . . . . . . . . 4-11 Open Loop vs. Closed-Loop with the AGS to BGYRO Controller 
4.2.5 BGYRO-Y Response to a BET-X Pulse:

Open Loop vs. Closed-Loop with the AGS to BGYRO Controller

4.2.6 DET-Y Response to a BET-Y Pulse: . . . . . . . . . . . . . . . . . . . . . 4-13

Open Loop vs. Closed-Loop with the AGS to BGYRO Controller

4.2.7 BGYRO-X Response to a BET-Y Pulse: . . . . . . . . . . . . . . . . . . . 4-14

Open Loop vs. Closed-Loop with the AGS to BGYRO Controller

4.3.1 Block Diagram for LMED Feedback Loops . . . . . . . . . . . . . . . . . . . 4-15

4.3.2 BGYRO-Y Response to a BET-X Pulse: . . . . . . . . . . . . . . . . . . . 4-16 Open Loop vs. Closed Loop with the LMED Controller

4.3.3 ACCEL-1X Response to a BET-X Pulse: . . . . . . . . . . . . . . . . . . . 4-17 Open Loop vs. Closed Loop with the LMED Controller

4.3.4 ACCEL-2X Response to a BET-X Pulse: . . . . . . . . . . . . . . . . . . . 4-18 Open Loop vs. Closed Loop with the LMED Controller

4.3.5 BGYRO-X Response to a BET-Y Pulse: . . . . . . . . . . . . . . . . . . . 4-19 Open Loop vs. Closed Loop with the LMED Controller

4.3.6 ACCEL-1Y Response to a BET-Y Pulse: . . . . . . . . . . . . . . . . . . . 4-20 Open Loop vs. Closed Loop with the LMED Controller

4.3.7 ACCEL-2Y Response to a BET-Y Pulse: . . . . . . . . . . . . . . . . . . . . 4-21 Open Loop vs. Closed Loop with the LMED Controller

5.1.3 DET-X Response to a BET-X Pulse: . . . . . . . . . . . . . . . . . . . . . 5-5 Open Loop vs. Closed Loop with the Integrated Controller

5.1.4 BGYRO-Y Response to a BET-X Pulse: . . . . . . . . . . . . . . . . . . . 5-6 Open Loop vs. Closed Loop with the Integrated Controller

5.1.5 ACCEL-1X Response to a BET-X Pulse: . . . . . . . . . . . . . . . . . . . 5-7 Open Loop vs. Closed Loop with the Integrated Controller

5.1.6 ACCEL-2X Response to a BET-X Pulse: . . . . . . . . . . . . . . . . . . . 5-8 Open Loop vs. Closed Loop with the Integrated Controller

5.1.7 DET-Y Response to a BET-Y Pulse: . . . . . . . . . . . . . . . . . . . . . 5-9 Open Loop vs. Closed Loop with the Integrated Controller 
5.1.8 BGYRO-X Response to a BET-Y Pulse: . . . . . . . . . . . . . . . . . . . 5-10 Open Loop vs. Closed Loop with the Integrated Controller

5.1.9 ACCEL-1Y Response to a BET-Y Pulse: . . . . . . . . . . . . . . . . . . . 5-11 Open Loop vs. Closed Loop with the Integrated Controller

5.1.10 ACCEL-2Y Response to a BET-Y Pulse: . . . . . . . . . . . . . . . . . . . 5-12 Open Loop vs. Closed Loop with the Integrated Controller

5.2.1 RCS Disturbance Profile . . . . . . . . . . . . . . . . . . . . . . . . 5-13

5.2.2 DET-X Response to a BET-X RCS Disturbance: . . . . . . . . . . . . . . . . 5-14 Open Loop vs. Closed Loop with the Integrated Controller

5.2.3 BGYRO-Y Response to a BET-X RCS Disturbance: . . . . . . . . . . . . . . 5-15 Open Loop vs. Closed Loop with the Integrated Controller

5.3.1 Crew Disturbance Profile . . . . . . . . . . . . . . . . . . . . . 5-16

5.3.2 DET-X Response to a BET-X Crew Disturbance: . . . . . . . . . . . . . . . 5-17 Open Loop vs. Closed Loop with the Integrated Controller 


\section{INTRODUCTION}

Many large space system concepts will require active vibration control systems to satisfy critical performance requirements such as line-of-sight (LOS) accuracy and constraints on rms surface roughness. In order for these concepts to become operational it is imperative that the benefits of active vibration control be practically demonstrated in ground-based experiments. This paper discusses an experiment conducted by Harris as part of the NASA CSI Guest Investigator Program. The results of this experiment, which has features directly traceable to future space missions, successfully demonstrate active vibration control for a flexible structure. The testbed, shown in Figure 2.1, is the ACES structure at NASA Marshall Space Flight Center (MSFC).

The primary objective of this experiment was to design controllers that provide substantial reduction of the LOS errors. The satisfaction of this objective required the controllers to significantly attenuate the beam vibration. Particular emphasis was also placed on controller simplicity (i.e., reduced-order and decentralized controller architectures). Complexity reduction in control law implementation is of paramount interest due to stringent limitations on throughput of even state-of-the-art space qualified processors.

The development of the system models used for control design was facilitated by using the Eigensystem Realization Algorithm developed at NASA [12-13]. As will be described more fully in Section 3 , the strong and weak interaction patterns in the dynamics of the ACES structure allowed us to approach the control design by designing decentralized control laws for selected system loops involving eight actuators and eight sensors.

The primary methodology chosen for control design in this experiment is the Optimal Projection Approach for Uncertain Systems (OPUS) [1-10]. The OPUS design process allows for the simultaneous trade-off of four fundamental issues in control design: actuator sizing, sensor accuracy, controller order and robustness versus system performance. A subset of OPUS is the Maximum Entropy/Optimal Projection (MEOP) methodology [2-6] which was developed particularly to allow high performance, robust control law design for flexible structures. The MEOP design equations (Figure 1.1) consist of four coupled matrix equations which specialize to the standard LQG Riccati equations when the plant is known perfectly and a full order controller is desired. In this experiment MEOP was used to develop control laws for active vibration suppression. The discrete-time optimal projection approach [7-8] was used to develop control laws for a tracking problem associated with two of the feedback loops. 
The paper is organized as follows. Section 2 describes the basic ACES configuration. Section 3 then discusses the finite element model provided by MSFC and the development of the models actually used for control design. This section also motivates the decentralized approach chosen for the control design. Next, Section 4 describes control design for eight system loops which were selected by analysis of test data collected from the structure. Section 5 then presents the experimental results. It is seen that very significant performance improvement is achieved when the eight feedback loops are closed. Finally, Section 6 presents closing remarks and conclusions. 


$$
\begin{aligned}
& 0=A_{s} Q+Q A^{\mathrm{T}}+V_{1}-Q \bar{\Sigma} Q+\tau_{\perp} Q \bar{\Sigma} Q \tau_{\perp}^{\mathrm{T}} \\
& +\sum_{i=1}^{P} \alpha_{i}^{2} A_{i} Q A_{i}^{\mathrm{T}}+\sum_{i=1}^{P} \alpha_{i}^{2} A_{i} \hat{Q} A_{i}^{\mathrm{T}} \\
& 0=A_{\mathrm{a}}^{\mathrm{T}} P+P A^{\mathrm{T}}+R_{1}-P \Sigma P+\tau_{\perp}^{\mathrm{T}} P \Sigma P \tau_{\perp} \\
& +\sum_{i=1}^{P} \alpha_{i}^{2} A_{i}^{\mathrm{T}} P A_{i}+\sum_{i=1}^{P} \alpha_{i}^{2} A_{i}^{\mathrm{T}} \hat{P} A_{i} \\
& 0=\left(A_{s}-\Sigma P\right) \hat{Q}+\hat{Q}\left(A_{s}-\Sigma P\right)+Q \bar{\Sigma} Q-\tau_{\perp} Q \bar{\Sigma} Q \tau_{\perp}^{\mathrm{T}} \\
& 0=\left(A_{s}-Q \bar{\Sigma}\right)^{\mathrm{T}} \hat{P}+\hat{P}\left(A_{s}-Q \bar{\Sigma}\right)+P \Sigma P-\tau_{\perp}^{\mathrm{T}} P \Sigma P \tau_{\perp} \\
& \operatorname{rank} \hat{Q}=\operatorname{rank} \hat{P}=\operatorname{rank} \hat{Q} \hat{P}=n_{c} \\
& \tau=\hat{Q} \hat{P}(\hat{Q} \hat{P})^{\#}, \quad(\cdot)^{\#} \text { denotes the group generalized inverse. }
\end{aligned}
$$

Figure 1.1 The MEOP design equations consist of four modified Riccati and Lyapunov equations, coupled by a projection matrix $\tau$ and allow high performance, robust control law developement for flexible structures. 

$-\infty-2$ 


\section{DESCRIPTION OF THE ACES STRUCTURE.*}

The ACES experimental testbed is located at NASA MSFC. The basic test article is a deployable, lightweight beam, approximately 45 feet in length. The test article is a spare Voyager Astromast built by ASTRO Research, Inc. It was supplied to MSFC by the Jet Propulsion Laboratory (JPL). The Astromast is extremely lightweight (about 5 pounds) and is very lightly damped.

The Astromast is a symmetric beam which is triangular in cross section. Three longerons form the corners of the beam and extend continuously along its full length. The cross members, which give the beam its shape, divide the beam into 91 sections each having equal length and mass and similar elastic properties. When fully deployed, the Astromast exhibits a longitudinal twist of approximately 260 degrees.

The ACES configuration (Figure 2.1) consists of an antenna and counterweight legs appended to the Astromast tip and the pointing gimbal arms at the Astromast base. The addition of structural appendages creates the "nested" modal frequencies characteristic of Large Space Structures (LSS). Overall, the structure is very flexible and lightly damped. It contains many closely spaced, low frequency modes (more than 40 modes under $10 \mathrm{~Hz}$ ). As illustrated by Figure 2.1, the ACES configuration is dynamically traceable to future space systems and is particularly responsive to the study of LOS issues.

The precise motion of the Base Excitation Table (BET) is obtained by supplying a commanded voltage input to the BET servo control system. The BET movements are monitored by a Linear Variable Differential Transformer (LVDT) whose outputs are fed back to the servo controllers. The servo controllers compare the commanded input voltage to the LVDT signals and automatically adjust the position of the BET. The closed-loop controller allows any type of BET movement within the frequency limitations of the hydraulic system. In this experiment the disturbances are chosen to be position commands to the BET.

The Image Motion Compensation (IMC) System consists of a 5-mW laser, two 12-inch mirrors, two pointing gimbals, a four quadrant detector and associated electronics, and two power supplies. Figure 1.1 shows the location of each of the components of the IMC system. The goal of the control design is to position the laser beam in the center of the detector. The detector and pointing gimbals are each positioned on the end of a flexible appendage to increase the difficulty of the

\footnotetext{
* This description of the ACES testbed is taken primarily from [11]
} 
control problem. The lack of information about the appendage motion also adds complexity to the controller design (i.e., there is no accelerometer or gyro at the location of the gimbals or the detector).

In addition to the two IMC gimbals, the available control actuators also include the Advanced Gimbal System (AGS), a precision, two-axis gimbal system designed for high accuracy pointing applications, which has been augmented with a third gimbal in the azimuth. The gimbal system provides torque actuation at the base of the Astromast. The AGS receives commands from the control algorithm (implemented on an HP 9000 via the COSMEC data acquisition system) in the form of analog inputs over the range of -10 to +10 volts. This saturation represents a current limit of 27 amps which is built into the AGS servo amplifier as a protective measure. Because the AGS servo amplifier outputs a current that causes an applied torque proportional to the current, the control algorithms used in the COSMEC must be designed to produce torque command signals.

The AGS gimbal torquers, with the power supply and servo amplifiers used in the SSC laboratory, can generate $37.5 \mathrm{ft}$-lbs of torque over an angular range of approximately \pm 30 degrees. The azimuth torquer is capable of generating $13.8 \mathrm{ft}$-lbs over an angular range of \pm 5 degrees. It can, however, be set manually to allow \pm 5 degrees of rotation at any position about the 360 degrees of azimuth freedom. This allows the test article to be rotated to any position desired without remounting.

Linear Momentum Exchange Devices (LMEDs) provide colocated sensor/actuator pairs which apply forces and measure the resulting accelerations. Each LMED package contains two LMEDs having orthogonal axes, two accelerometers, and two Linear Variable Differential Transformers (LVDT's). The two LMED packages are positioned at intermediate points along the Astromast. These locations were selected to maximize the ability of these devices to control the dominant structural modes. When the Astromast is at rest, each LMED package is aligned with the $\mathrm{X}$ and $\mathrm{Y}$ axes of the inertial reference frame shown in Figure 2.1. The LMED applies a horizonal force to the structure and a colocated accelerometer measures the resulting acceleration at the actuator location.

The LMED is linear permanent magnet motor whose magnet functions as a proof mass. Force is applied to the structure as a reaction against the acceleration of proof mass. The magnet assembly travels along a single shaft on a pair of linear bearings. The armature of the motor is of a hollow coil which extends inside the magnet assembly from one end. The magnet assembly moves along 
the shaft with respect to the coil which is fixed to the LMED package. The magnet is constrained on each end by a bracket which provides a small centering force to the proof mass. A linear accelerometer is mounted in line with the shaft. An LVDT is utilized to measure the position of the proof mass with respect to the LMED assembly.

In addition to the two-axis detector associated with the IMC System and the accelerometers and LVDT's associated with the LMEDs, the available measurement devices include three-axis rate gyros at the tip and base as well as three-axis accelerometers at the tip and base. However, since the three-axis rate gyros at the tip are not available for controller implementation and were not used for evaluation, we will describe only the remaining measurement devices.

The rate gyros at the base are Apollo Telescope Mount (ATM) Rate Gyros. They are designed to measure small angular rates very precisely. The analog output signals of an ATM rate gyro package is \pm 45 volts. The analog signal is converted to 12-bit binary words by the analog-todigital converter card of the COSMEC system. The ATM rate gyro packages require a warmup period of approximately 40 minutes. Each package requires 1.5 amps during warmup and then 1.25 amps after stabilization, both at 28 volts DC.

The accelerations at the base and tip of the ASTROMAST are measured by two identical three-axis accelerometer packages. The accelerometers provide resolution finer than $0.0001 \mathrm{~g}$ and a dynamic range of $\pm 3 \mathrm{~g}$ with a bandwidth of 25 to $30 \mathrm{~Hz}$. They require approximately 20 minutes for warmup, during which time each package requires 1.2 amps at 28 Volts DC. After warmup the power requirement reduces to about $0.9 \mathrm{amp}$ per package. The accelerometer electronics are included on board the instrument package.

The signals from the accelerometers are different from the ATM rate gyros. Two channels are required for each degree of freedom of the accelerometer package, i.e., six channels per accelerometer package. One channel of each pair carries a $2.4-\mathrm{kHz}$ square wave synchronization signal, and the other channel carries the acceleration information. Zero acceleration is represented by a signal identical to that of the synchronization channel, positive acceleration by an increase in frequency, and negative acceleration by a decrease in frequency as compared to the synchronization channel. As in the cases of the other instruments, these signals are monitored by a hardware card in the COSMEC system.

As mentioned previously the computer system consists of an HP 9000 digital computer inter- 
faced with the COSMEC Input/Output system. The HP 9000 performs the control algorithm, data storage, real-time plotting, and the strapdown algorithm (described in the next section). The HP 9000 is a 32-bit machine with an $18-\mathrm{MHz}$ clock rate. It includes an HPIB interface card, two 16-bit parallel interface cards, 512 kbytes of extra memory, and a floppy disc drive. The benchmark test times for processing the present control and strapdown algorithms, plotting, and storage are .010 to .013 milliseconds per sample.

The COSMEC is a highly modified AIM-65 microcomputer system used for I/O processing. The primary purposes of the COSMEC are to process the sensor inputs, to provide force and torque commands for the actuators, and to off-load control and sensor data to the computer system. Currently, the COSMEC performs these tasks with 25 sensor inputs and nine actuator outputs, while maintaining a 50-Hz sampling rate. The cycle time for COSMEC operation is approximately 5 milliseconds.

In our control design and implementation we used 8 control inputs and 8 measurement outputs. The inputs were the $\mathrm{X}$ and $\mathrm{Y}$ torques of the IMC gimbals, the $\mathrm{X}$ and $\mathrm{Y}$ torques of the AGS gimbals and the $\mathrm{X}$ and $\mathrm{Y}$ forces of the two LMED packages. The measurements consisted of the $\mathrm{X}$ and $\mathrm{Y}$ detector (DET) position outputs, the $\mathrm{X}$ and $\mathrm{Y}$ base gyro (BGYRO) rate outputs and the $\mathrm{X}$ and $\mathrm{Y}$ outputs of the LMED accelerometers. 


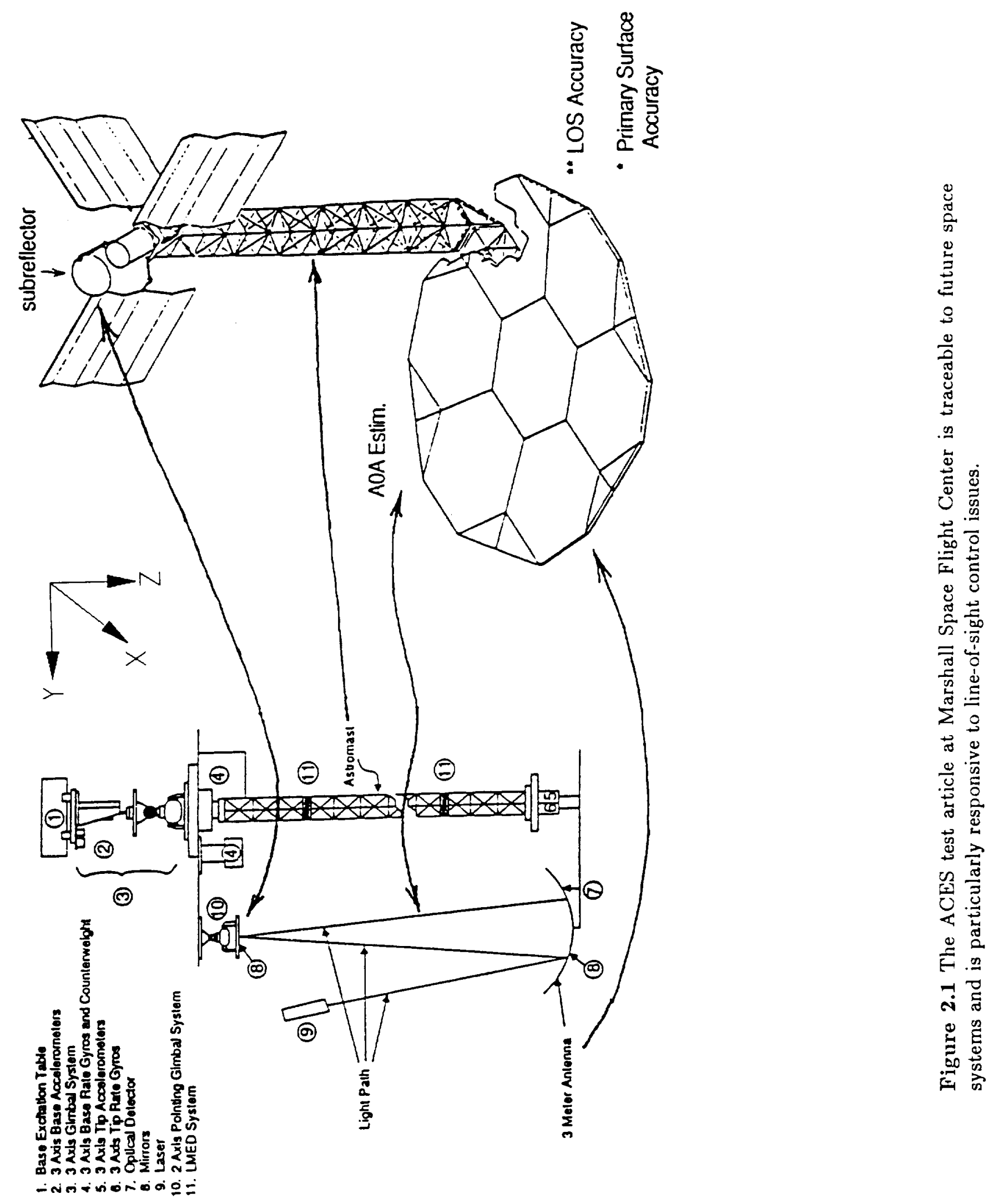


$-$ 


\section{MODELING PROCEDURE FOR THE ACES STRUCTURE}

The first critical step in control design is model development and validation. Below, we describe the process that led us to of develop our control design models by using a system identification procedure that is based upon the Eigensystem Realization Algorithm. We then present details of the system identification procedure.

\subsection{Choosing the Modeling Procedure}

Initial model development for flexible structures is usually based on the finite element approach. The initial finite element model (FEM) is evaluated by comparing its time and frequency responses with information gleaned from input-output data collected from the actual physical apparatus. If this comparison shows that the FEM is inadequate for control system design, then the control designer has essentially three options: (i) modify the FEM, (ii) develop a new model based on the input-output test data using an appropriate system identification algorithm or (iii) develop a model which is in some sense a hybrid of the FEM and models developed using system identification techniques.

In this experiment the inputs to the system were chosen to be either broad-band random noise, sine-sweeps or delta-functions. The inputs that yielded the most information about the system modes were the sine-sweeps and delta-functions. We conjecture that this is primarily due to limitiations on the length of the time-histories allowed and the fact that the broad-band random inputs did not significantly excite the dominant lower frequency dynamics in the allowable time windows. The input-output test data was used to generate frequency response functions (FRF's) of selected system loops and these FRF's were compared with the corresponding bode plots of the finite element model. Figures 3.1.1-3.1.5 show this comparison for four system loops: (i) IMC-X to DET-Y, (ii) IMC-Y to DET-X, (iii) AGS-X to BGYRO-X, and (iv) AGS-Y TO BGYRO-Y.

As evidenced by the comparisons of Figures 3.1.1-3.1.5, the FEM predicted significantly different frequency responses than those obtained by actually testing the structure. For example, Figure 3.1.1 shows that for the AGS-X to BGYRO-X loop the finite element model Bode plot does not contain the $1.4 \mathrm{~Hz}$ mode which most influenced the open loop LOS performance. The FEM's also do not show the large magnitude peaks corresponding to some of the modes past $8 \mathrm{~Hz}$. Figure 3.1 .2 shows analogous trends for the AGS-Y to BGYRO-Y loop. Also notice that as seen by Figure 3.1.3 the FEM predicts positive real behavior for the AGS-X to BGYRO-X loop, while the FRF reveals 
that between $2 \mathrm{~Hz}$ and $4 \mathrm{~Hz}$ the phase lags $-90^{\circ}$ by as much as $25^{\circ}$ (even when the computational delay due to the $50 \mathrm{~Hz}$ sample rate is not taken into account). This phase lag is probably due to actuator and sensor dynamics.

The FRF's of the IMC-X to DET-Y and IMC-Y to DET-X loops, shown respectively in Figures 3.1.4 and 3.1.5, reveal that these loops are influenced very little by the flexible modes of the structure. It follows that the IMC gimbals are not capable of controlling flexible modes to improve LOS performance. Thus, if one considers the four actuator inputs (IMC-X, IMC-Y, AGS-X and AGS-Y) and the four sensor outputs (DET-X, DET-Y, BGYRO-X, BGYRO-Y), it is not necessary to feed back the BGYRO outputs to the IMC gimbals since the BGYRO's primarily contain information about the behavior of the flexible modes which the IMC's cannot control. In addition, the DET outputs do not contain much (if any) useful information for the AGS gimbals that is not already provided by the BGYRO's. Thus, the achievable performance cannot be improved by feeding back the DET outputs to the IMC gimbals or the BGYRO outputs to the AGS gimbals. As illustrated by Figure 3.1.6, analysis of test data also revealed that within the decentralized structure described above there were four dominant loops: AGS-X to BGYRO-X, AGS-Y to BGYRO-Y, IMC-X to DET-Y, and IMC-Y to DET-X.

In summary, analysis of the test data revealed that for the four sensors and actuators described above the achievable performance with a decentralized control structure involving four dominant loops comparable to the achievable performance with a centralized control structure. Also, the analysis revealed that although the finite element model showed some of the trends seen in the FRF's generated by the test data and was thus adequate for preliminary design studies, the FEM was inadequate as a model for high performance control system design. Thus, it was necessary to either modify the FEM or develop a model based upon actual test data. Modifying the finite element model and accounting for the sensor and actuator dynamics would have been a very time-consuming and expensive process and thus we decided to use the Eigensystem Realization Algorithm (ERA) to develop state space models of the four dominant loops. It is important to note that since all test data were collected by using the control sensors and actuators, models generated from this data should account for the sensor and actuator dynamics.

We had difficulty obtaining useful input-output data from the LMED's due to the stroke limitations of their proof masses. In addition, early in the project the internal control configuration of the LMED's was in transition. In particular, MSFC in joint consultation with the guest inves- 
tigators decided to have the springs removed from the LMED's and replaced by internal position loops. The uncertainty regarding the dynamics of the LMED's led us to delay control design activities involving these devices. Ultimately, it was decided to simply feed back the colocated LMED accelerometers to the corresponding LMED force axis and to determine simple dynamics for these controllers by using crude models of the loops, developed by information provided to us by MSFC and our knowledge of proof mass devices. As will be seen in Section 5, these colocated LMED loops did provide significant performance improvement.

\subsection{Model Development Using the Eigensystem Realization Algorithm}

The collection and manipulation of test data to obtain high fidelity models for control design is usually an iterative procedure as illustrated by Figure 3.2.1. Figures 3.2.2 and 3.2.3 show the final steps in developing the models for the four major loops. Note that first-order all-pass filters were incorporated into the AGS to BGYRO loops to account for the computational delay. The control design models for the loops AGS-X to BGYRO-X and AGS-Y to BGYRO-Y were respectively 17 th and 19th order continuous-time models. The control design models for the loops IMC-X to DET-Y and IMC-Y to DET-X were both 4th order discrete-time design models. Ideally, for digitial implementation of a control algorithm it is better to design the controller directly using discretetime representations of the system model. We chose to design the AGS to BGYRO feedback loops using continuous-time models because of a greater maturity in the theory and software development for the continuous-time setting.

Figures 3.2.4-3.2.8 show comparisons of the ERA models of the four system loops with the FRF's generated from the test data. The magnitude plots of Figures 3.2.4, 3.2.5, 3.2.7, and 3.2.8 show that the ERA models closely resemble the FRF's. As illustrated by Figure 3.2.6, the all-pass filters were effective in emulating the computational delay in the system. 

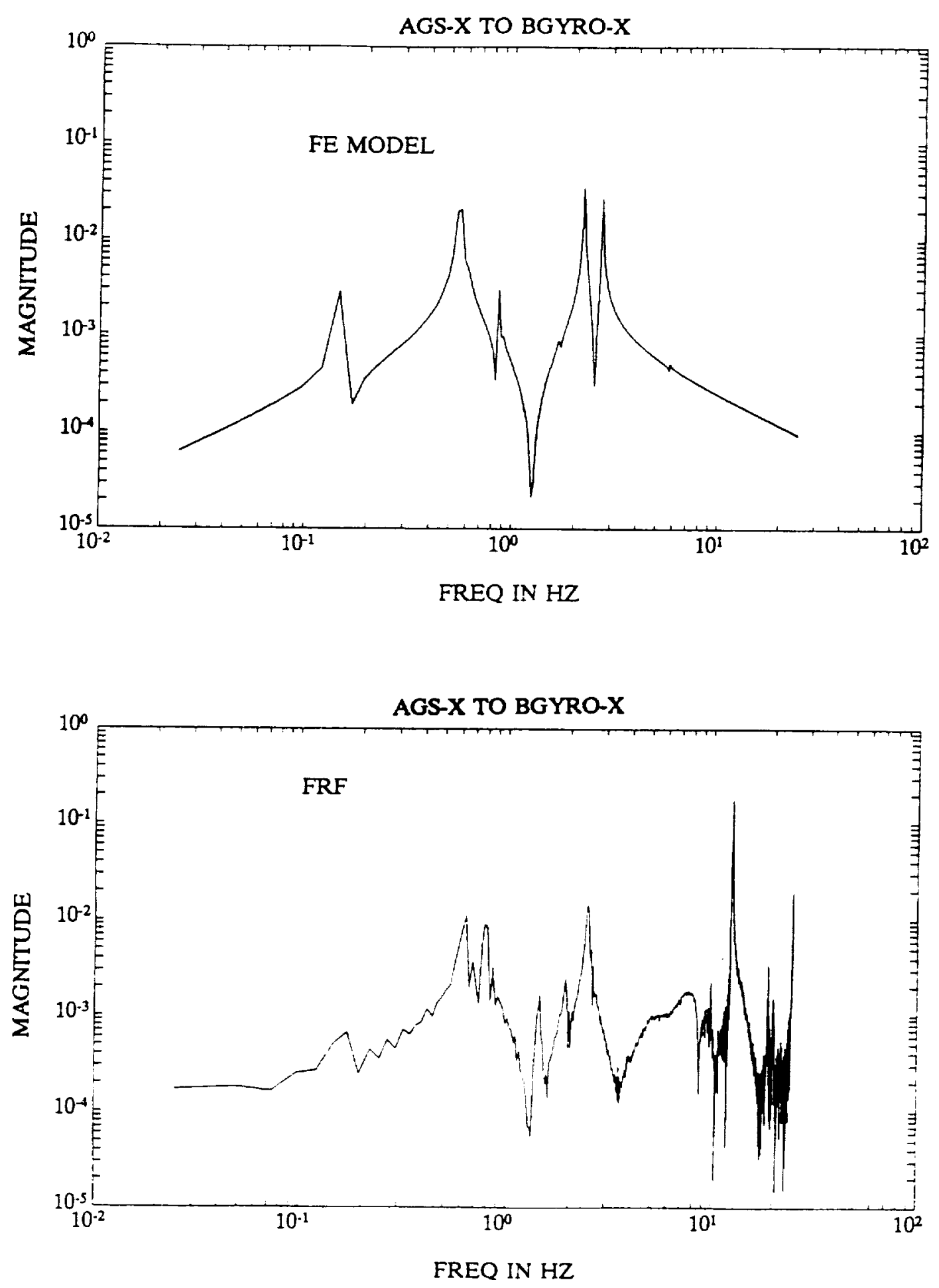

Figure 3.1.1 A comparison of the FRF data and finite element Bode plot for the AGS-X to BGYRO-X loop shows that the finite element model neglects the contribution of the $1.4 \mathrm{~Hz}$ mode (which most influences LOS performance) and does not show the large magnitude peaks corresponding to some of the higher frequency modes. 

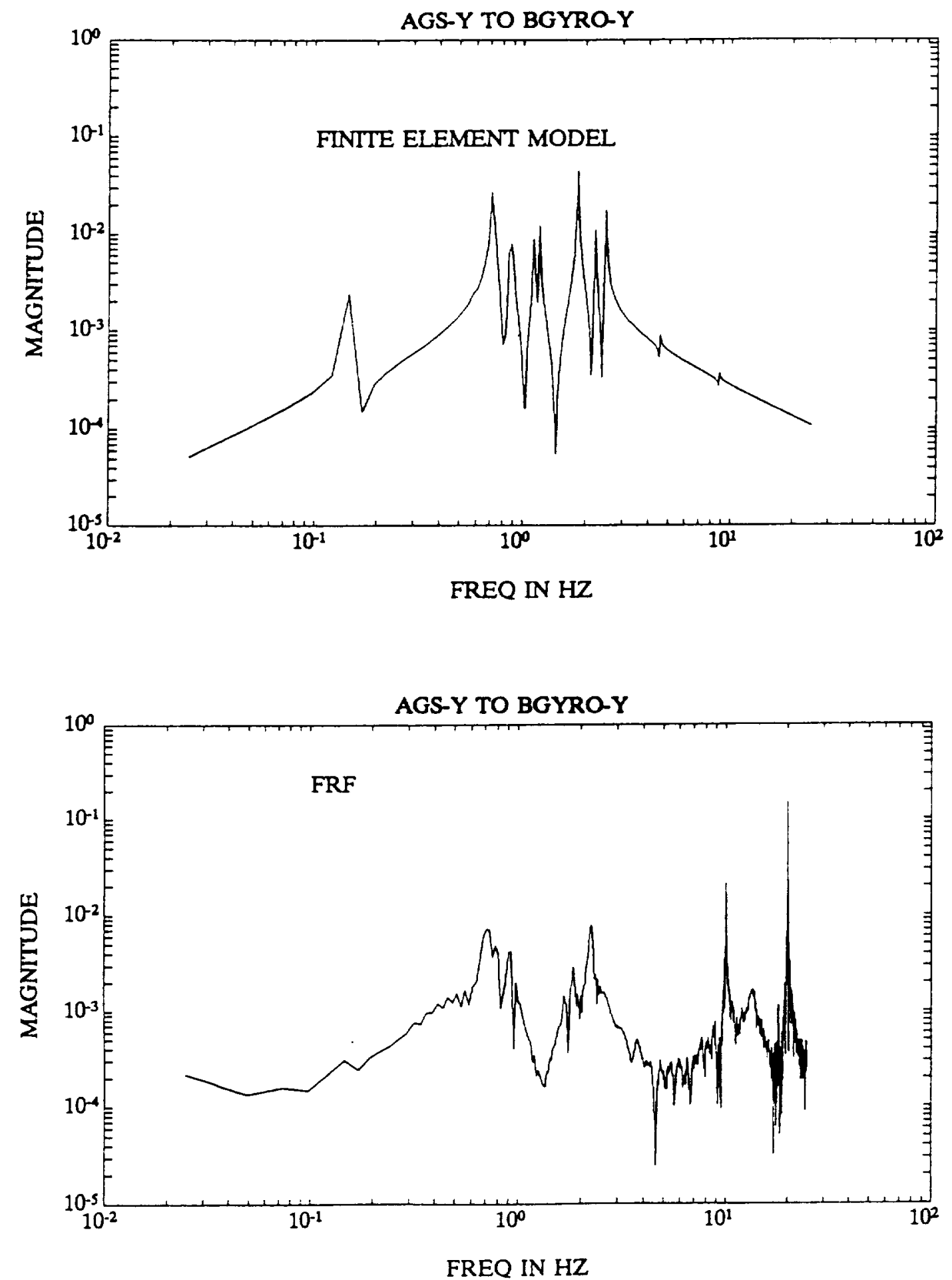

Figure 3.1.2 A comparison of the FRF data and finite element Bode plot for the AGS-Y to BGYRO-Y loop shows that the finite element model neglects the contribution of the $1.7 \mathrm{~Hz}$ mode (which most influences LOS performance) and does not show the large magnitude peaks corresponding to some of the higher frequency modes. 

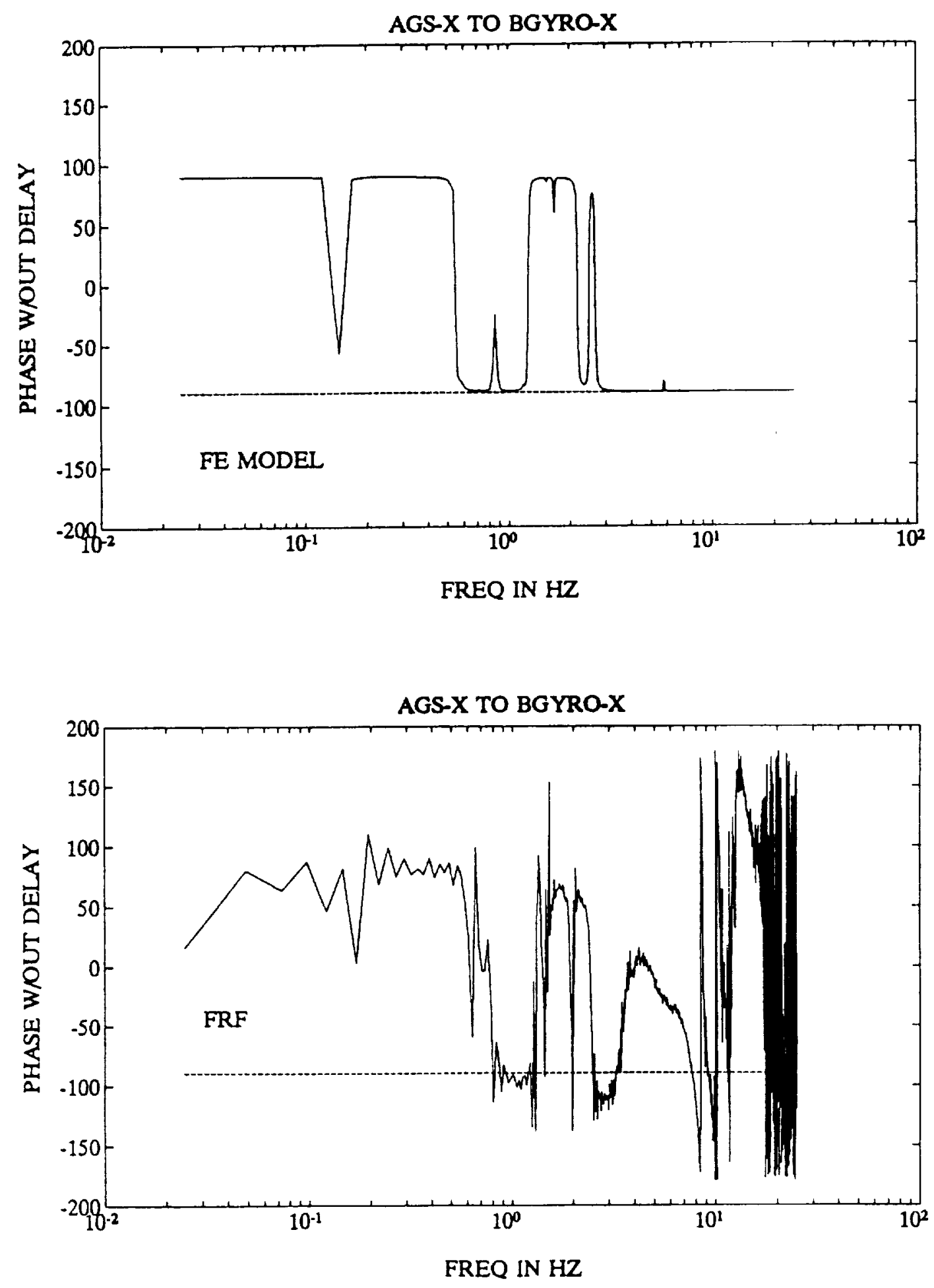

Figure 3.1.3 For the AGS-X to BGYRO-X loop the finite element loop predicts positive real behavior while the FRF reveals that between $2 \mathrm{~Hz}$ and $4 \mathrm{~Hz}$ the phase lags $-90^{\circ}$ by as much as $25^{\circ}$. 


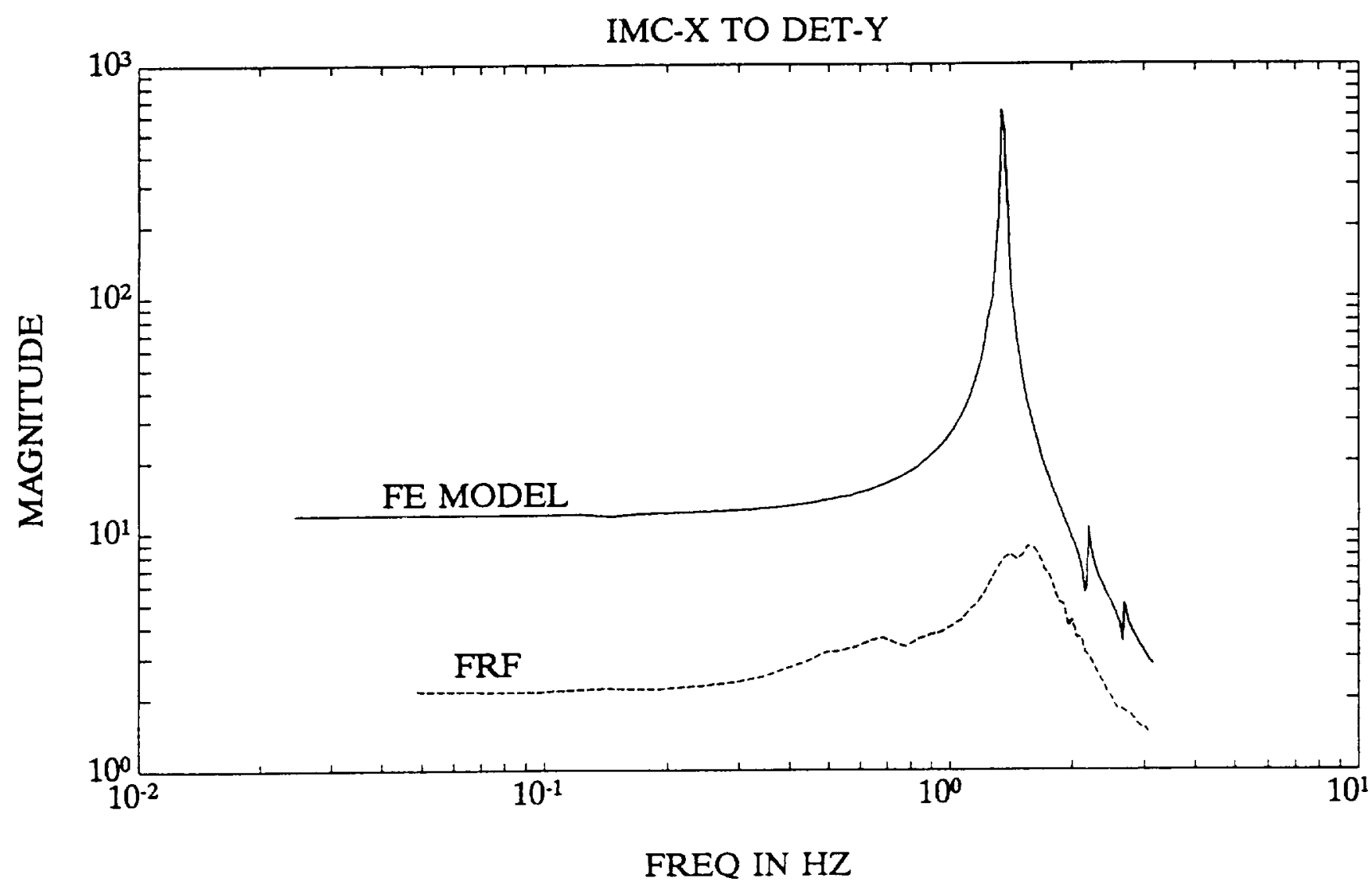

Figure 3.1.4 A comparison of the FRF data and finite element Bode plot for the IMC-X to DET-Y loop shows that the finite element model correctly predicts the small influence of the flexible modes and the dominance of the IMC-X mode but predicts much lower damping in the gimbal mode and much higher loop gain. 


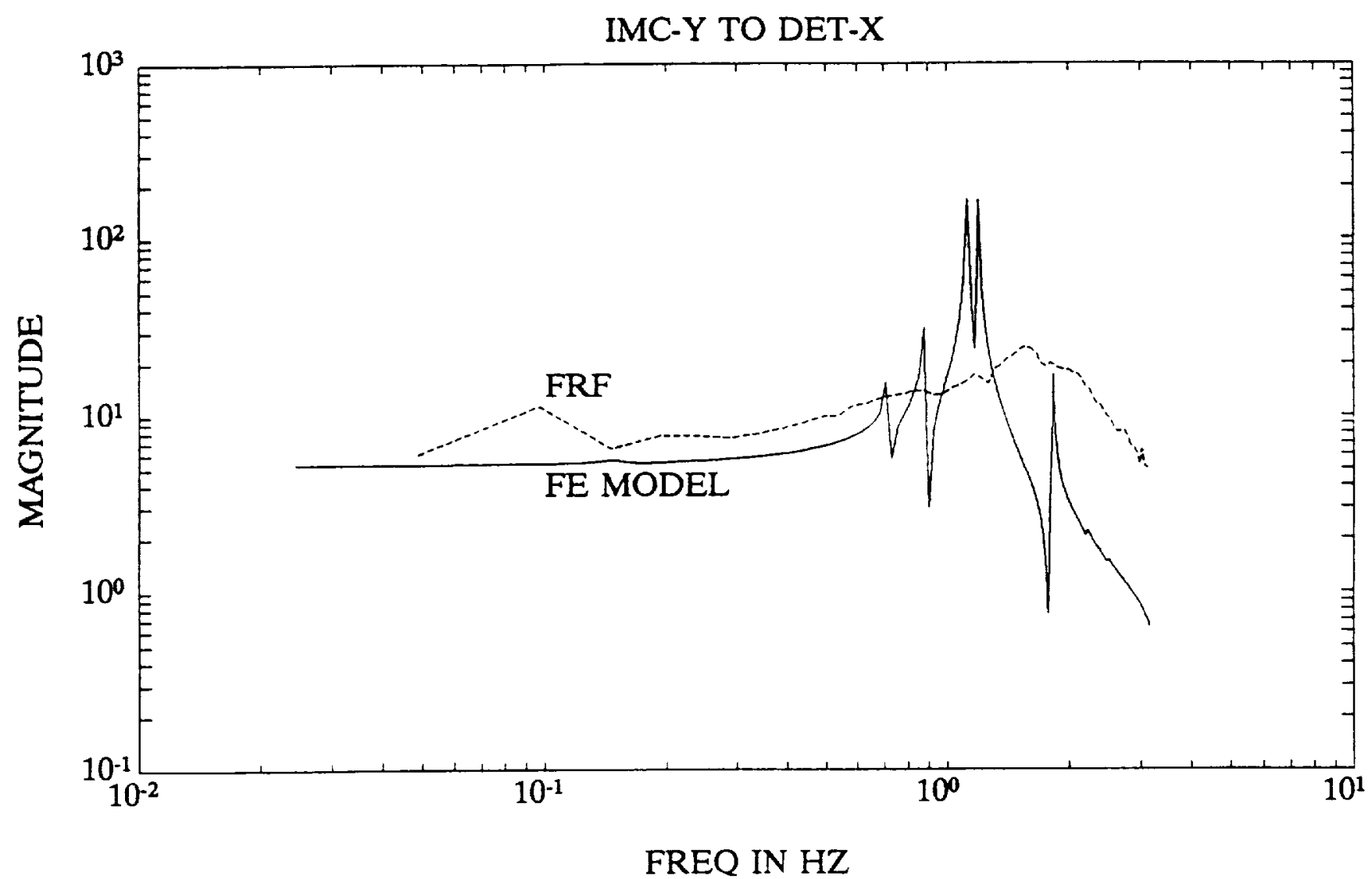

Figure 3.1.5 A comparison of the FRF data and finite element Bode plot for the IMC-Y to DET-X loop shows that the finite element model over estimates the influence of the flexible modes. 


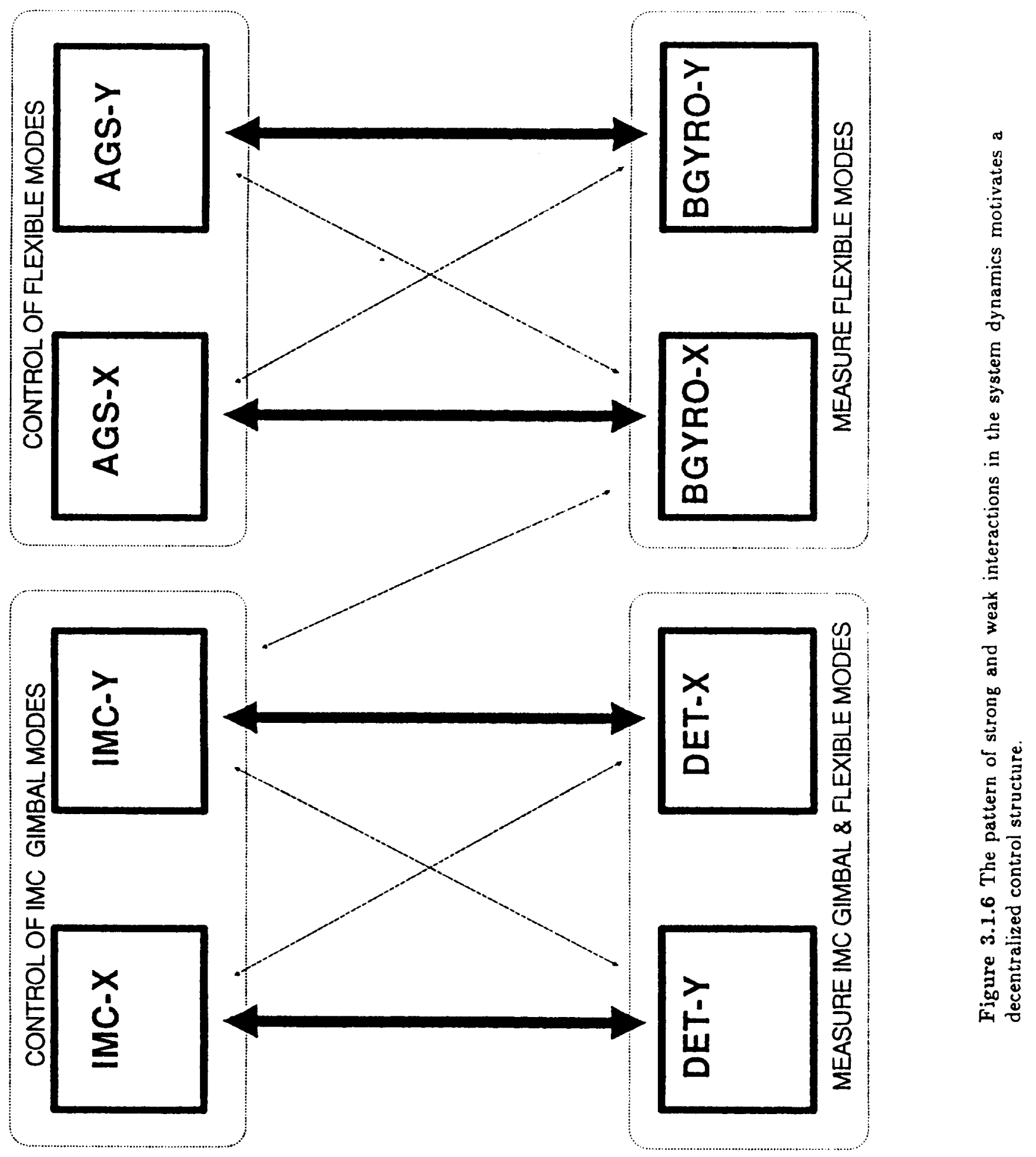




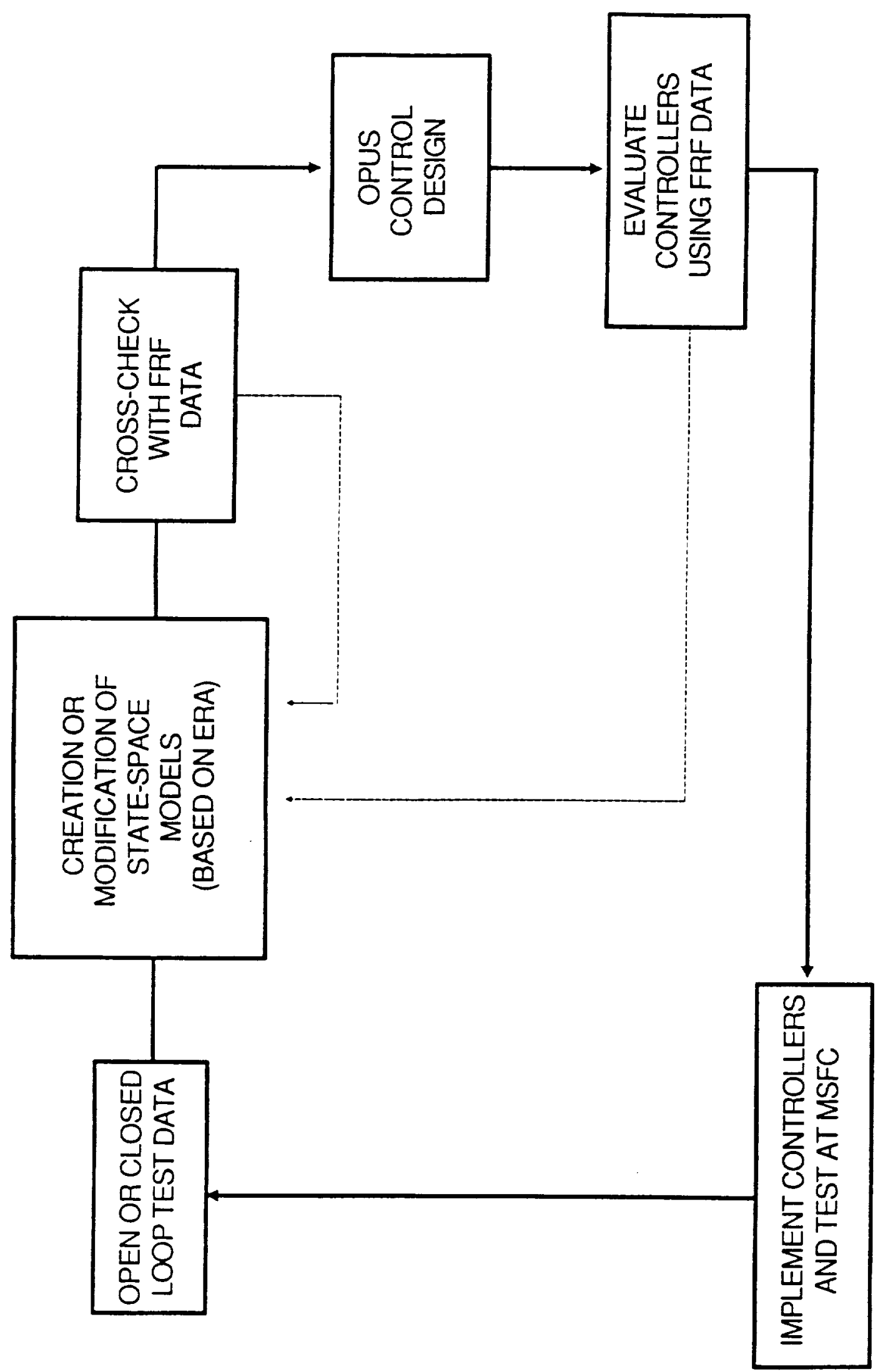


(I) AGS- $X$ TO BRATE- $X$

13 ERA STATES

-1 STATE LOST IN CONVERTING FROM DISCRETE-TIME TO CONTINUOUS-TIME

+4 STATES FOR HIGHER FREQUENCY UNMODELED MODES

+1 STATE FOR ALL-PASS TO EMULATE COMPUTATIONAL DELAY

$17^{\text {th }}$ ORDER CONTINUOUS-TIME DESIGN MODEL

(IV) AGS-Y TO BRATE- $Y$

17 ERA STATES

-1 STATE LOST IN CONVERTING FROM DISCRETE-TIME TO CONTINUOUS-TIME

-2 STATES FOR DELETED HIGH FREQUENCY MODE

+4 STATES FOR HIGHER FREQUENCY UNMODELED MODES

+1 STATE FOR ALL-PASS TO EMULATE COMPUTATIONAL DELAY

$19^{\text {th }}$ ORDER CONTINUOUS-TIME DESIGN MODEL

Figure 3.2.2 The final steps in developing control design models for the AGS-X to BGYRO-X and AGS-Y to BGYRO-Y loops yielded respectively 17 th and 19 th order continuous-time models. 
(I) IMC-X TO DET-Y

6 ERA STATES (DISCRETE-TIME)

-4 SPURIOUS STATES

+1 DELAY STATE

+1 FILTER DISTURBANCE STATE

$4^{\text {th }}$ ORDER DISCRETE-TIME DESIGN MODEL

(II) IMC-Y TO DET-X

USED IMC- $X$ TO DET- $Y$ DESIGN MODEL

(THE OPEN LOOP GAIN AND THE DAMPING OF

THE DOMINANT MODE WAS MODIFIED, HOWEVER)

Figure 3.2.3 The final steps in developing control design models for the IMC-X to DET-Y and IMC-Y to DET-X loops yielded 4 th order discrete-time models. 

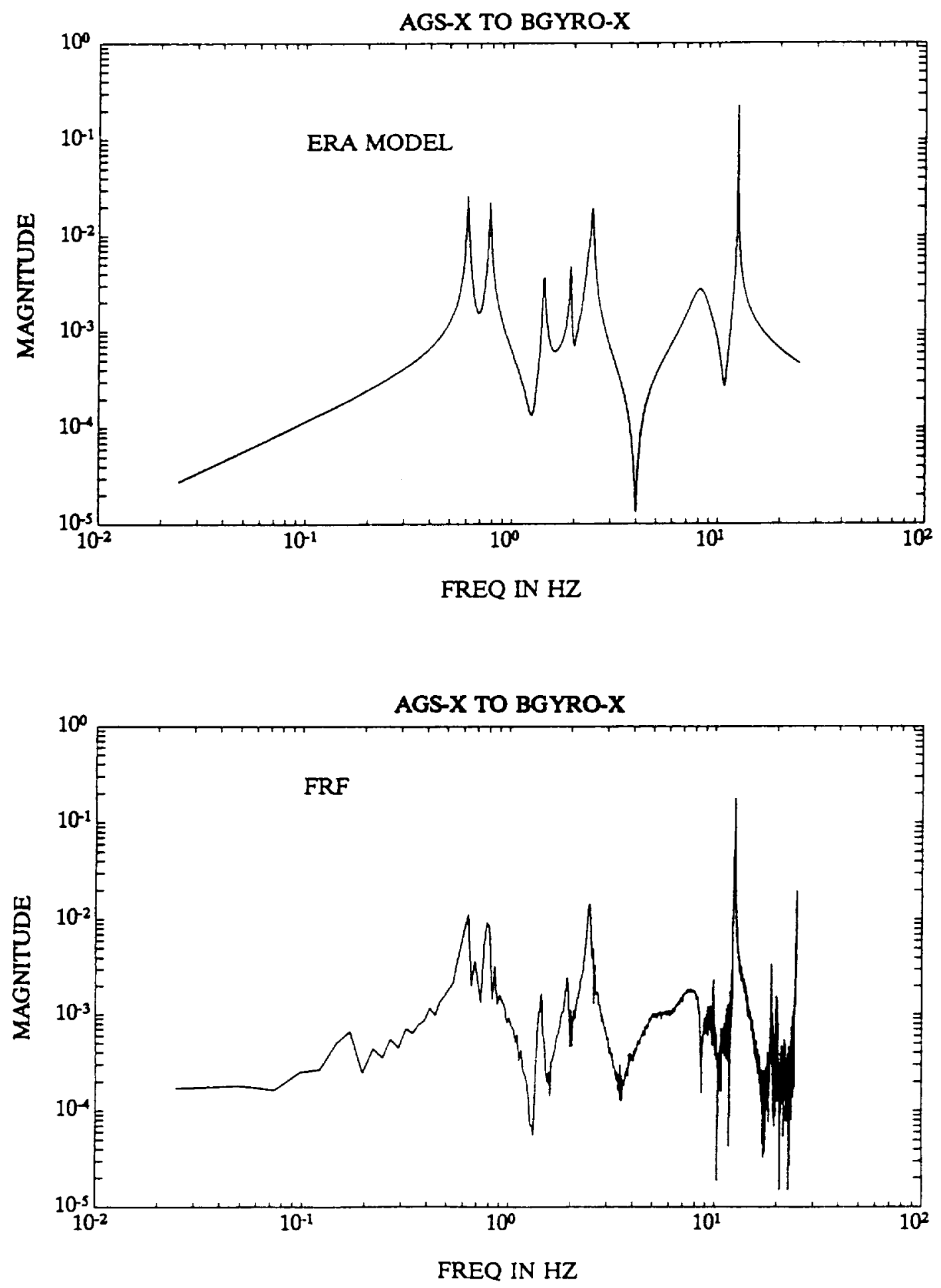

Figure 3.2.4 The ERA model for the AGS-X to BGYRO-X loop closely resembles the FRF generated from test data. 

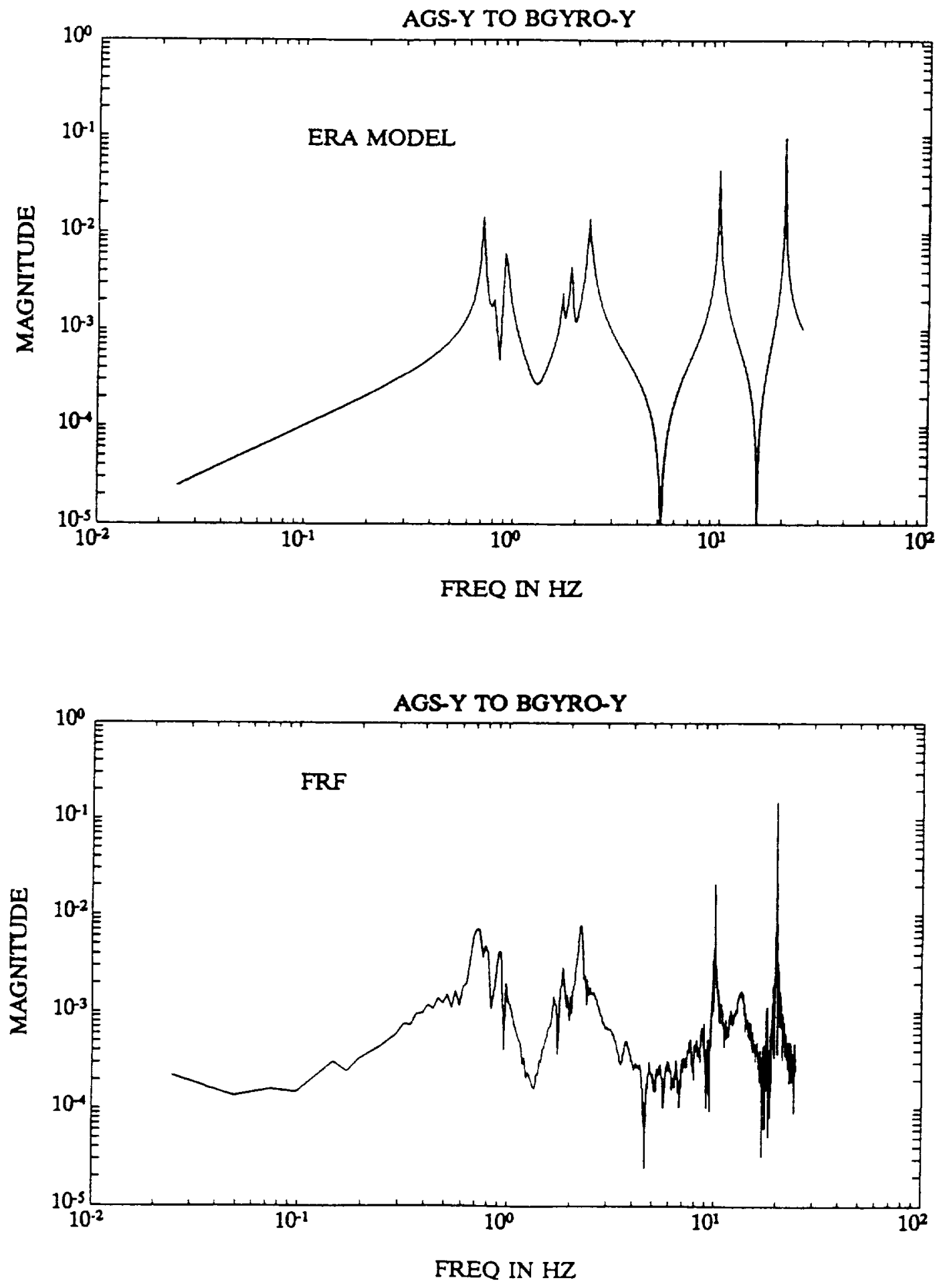

Figure 3.2.5 The ERA model for the AGS-Y to BGYRO-Y loop closely resembles the FRF generated from test data. 

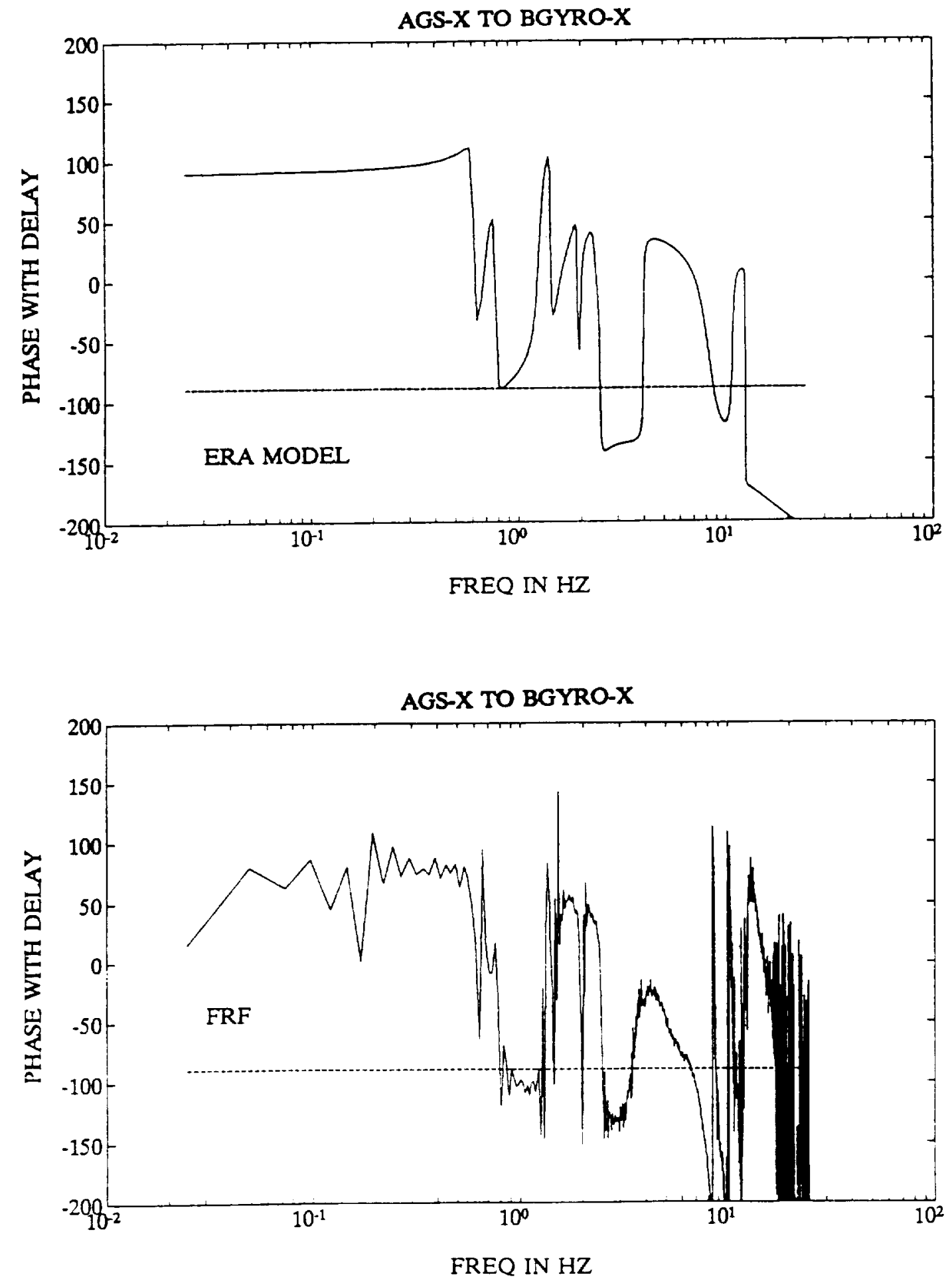

Figure 3.2.6. As shown here for the AGS-X to BGYRO-X loop, the ERA models effectively modeled the computational delay by using all-pass filters. 


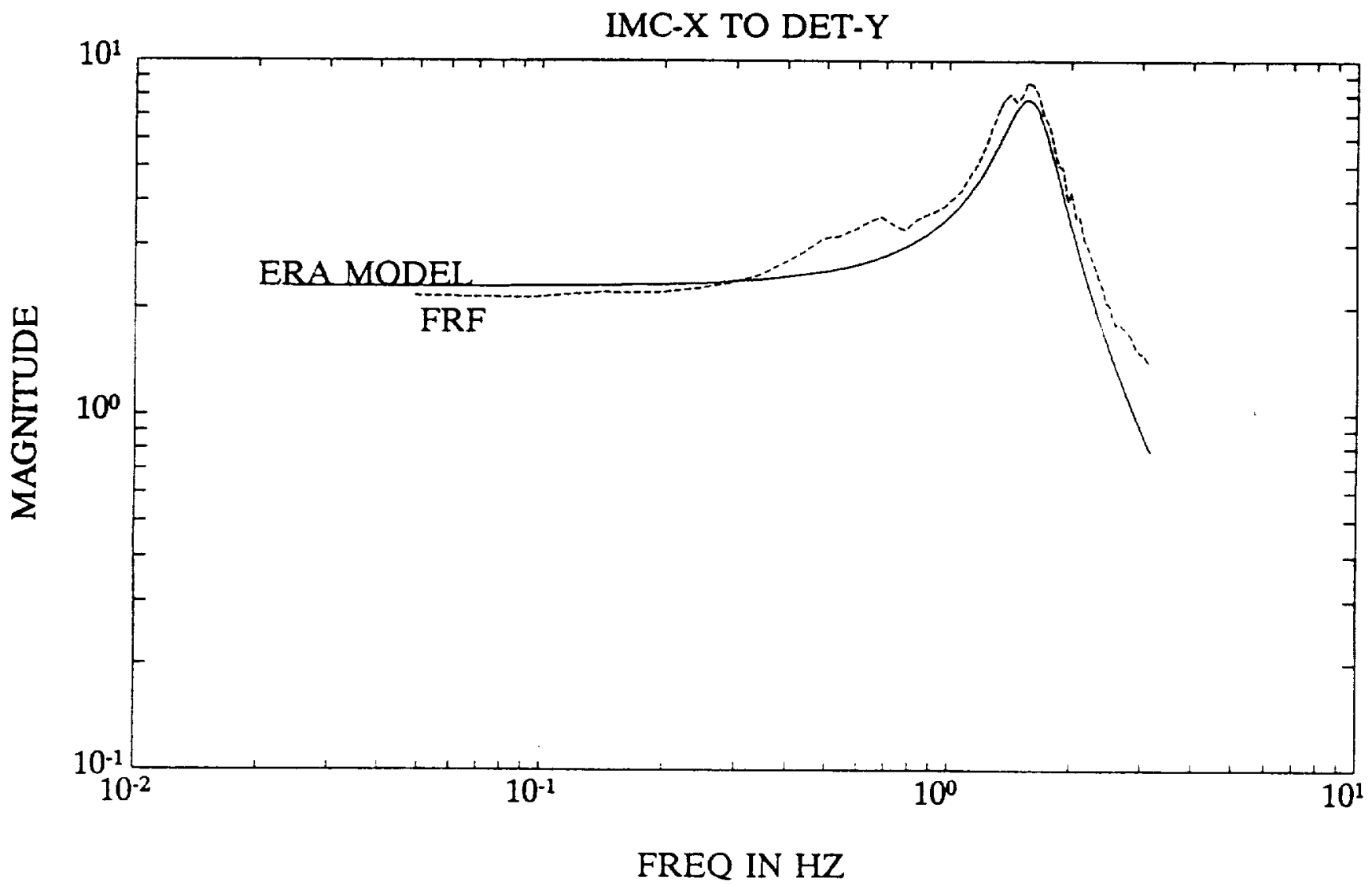

Figure 3.2.7 The ERA model for the IMC-X to DET-Y loop closely resembles the FRF generated from test data. 


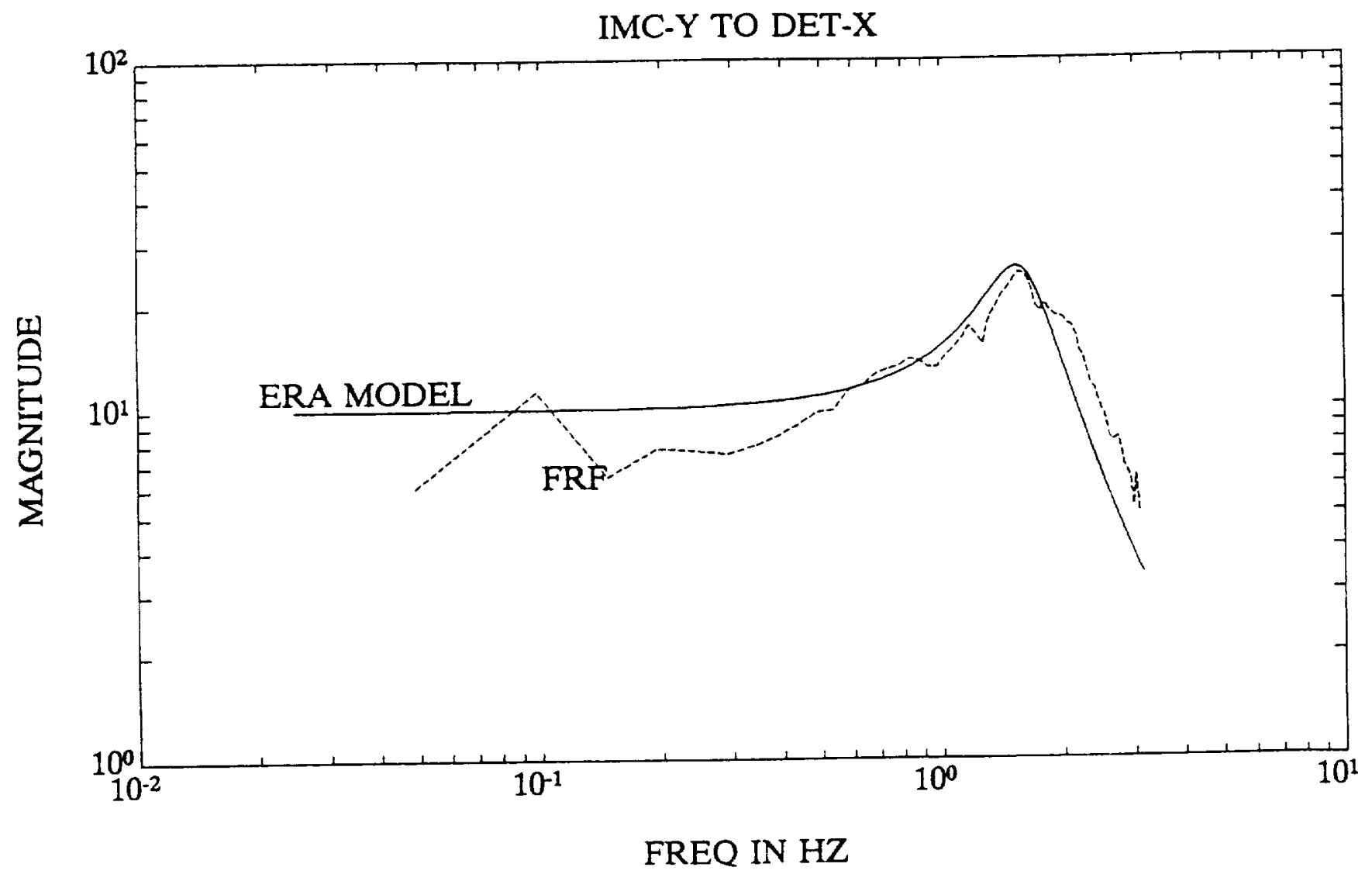

Figure 3.2.8 The ERA model for the IMC-Y to DET-X loop closely resembles the FRF generated from test data. 



\section{CONTROL DESIGN FOR THE ACES STRUCTURE}

Once we had settled on a decentralized controller architecture, control design for the ACES structure was essentially a three step process:

(i) design of the IMC gimbal to detector loops,

(ii) design of the AGS gimbal to base gyro loops, and

(iii) design of the LMED force to colocated accelerometer loops.

Below, we give details of the design procedures for each step along with experimental data describing the resultant performance improvement. Experimental data describing the performance improvement of the integrated controller (i.e., the controller with all feedback loops closed) is delayed until Section 5. The sample rate for each of the three feedback paths was $50 \mathrm{~Hz}$.

\subsection{Design Process for the Detector to IMC Loops}

The design processes for the IMC-X to DET-Y loop and the IMC-Y to DET-X loop were essentially identical. As illustrated by Figure 4.1.1, for each loop the control problem was formulated as a disturbance tracking problem. The disturbance to be tracked $w_{1}^{\prime}$ was modeled by filtering a white noise process $w_{1}$ through a filter $1 /(z-1+\varepsilon)$, which approximates a discrete-time integrator as the positive parameter $\varepsilon$ approaches zero. This filter accounted for the system biases and also the low frequency modes (i.e., modes below $1.5 \mathrm{~Hz}$ ). Optimal Projection control laws $H(z)$ were synthesized [7-8] by minimizing the quadratic cost function

$$
J(\varepsilon)=\lim _{k \rightarrow \infty} E\left[q^{\mathrm{T}}(\varepsilon) q(\varepsilon)+\rho u^{\mathrm{T}}(\varepsilon) u(\varepsilon)\right], \quad \rho>0
$$

The controllers implemented were the limiting controllers as $\varepsilon \rightarrow 0$. Thus, the implemented controllers contained integrators which were able to effectively eliminate the line-of-sight biases. For both loops the design models were 4 th order while the controllers implemented were 3 rd order. The controller gains are given in Appendix A.

The resultant performance improvement is illustrated by Figures 4.1.2-4.1.3 which show openloop and closed-loop responses of the $\mathrm{x}$ and $\mathrm{y}$ axes of the detectors respectively to pulse commands to the $\mathrm{x}$ and $\mathrm{y}$ axes of the Base Excitation Table. Notice that the feedback loops were able to eliminate the system biases and also improved the LOS performance by providing low frequency tracking. 


\subsection{Design Process for the Base Gyro to AGS Gimbal Loops}

The design processes for the AGS-X to BGYRO-X loop and the AGS-Y to BGYRO-Y (subsequently to be referred to as the $\mathrm{X}$ and $\mathrm{Y}$ loops) were very similar. As mentioned in Section 3 , the design model for the $\mathrm{X}$-loop was a 17 th order continuous-time model while the design model for the Y-loop was a 19th order continuous-time model. For the X-loop the modes that most influenced LOS performance were $1.4 \mathrm{~Hz}$ and $2.4 \mathrm{~Hz}$ bending modes. Similarly, for the Y-loop the modes that most influenced LOS performance were $1.7 \mathrm{~Hz}$ and $2.3 \mathrm{~Hz}$ bending mode.

Maximum Entropy Optimal Projection (MEOP) synthesis was used to synthesize continuoustime controllers of varying orders, control authorities and robustness. These controllers were then discretized by using the bilinear transformation with frequency prewarping. In our control designs we penalized only the modes less than $3 \mathrm{~Hz}$ since these modes dominate the open-loop LOS performance. The Maximum Entropy (ME) robustness design proved to be crucial in developing stable controllers which yielded significant performance improvement when implemented. The utility of the ME approach is illustrated by Figures 4.2.1-4.2.3.

Figure 4.2.1 describes the influence of ME uncertainty design on the phase of a full-order compensator in the performance region (i.e., less than $3 \mathrm{~Hz}$ ). The phase of the LQG compensator varies widely over this frequency interval, implying that the Nyquist plot of the corresponding loop transfer function encircles the origin several times. As one would expect, these designs were nonrobust and were unstable when implemented. However the ME designs became positive real in the performance region tending toward rate feedback. Thus the ME designs provided the needed stability robustness in the performance region.

Figure 4.2.2 describes the influence of ME uncertainty design on the magnitude shape of a full-order compensator in the performance region. Notice that the $\mathrm{ME}$ compensator magnitudes are smoother than those of the LQG compensators, thus providing performance robustness. Another implication is that the ME designs yield robust controllers that are effectively reduced order controllers. In practice, the full-order ME designs actually provide insight into the choice of the order of the compensator and is a numerical aid in synthesizing reduced order controllers.

The higher authority controllers notched the high frequency modes that had high gain, i.e., the two highest frequency modes shown in the ERA models of Figures 3.2.4 and 3.2.5. As illustrated by Figure 4.2.5, ME design was able to robustify the controller notches. That is, the controller 
notches were increased in both width and depth.

The controllers which yielded the best performance when implemented were a 4th order controller for the X-loop and a 6 th order controller for the Y-loop. The controller gains are presented in Appendix A.

The resultant performance improvement is shown in Figures 4.2.4-4.2.7 which show open and closed loop responses of the detectors and base gyros to pulse commands to the $\mathrm{x}$ and $\mathrm{y}$ axes of the Base Excitation Table. Notice that significant performance improvement was achieved in both the detector and base gyro responses

\subsection{Design Process of the LMED Force to Accelerometer Loops}

In this subsection and hereafter the two-axis LMED device closest to the base will be referred to as LMED-1 while the two-axis LMED device closest to the tip will be referred to as LMED-2. The control design was based on feeding back each of the four colocated accelerometer outputs to the corresponding LMED proof-mass axis. Thus the four loops utilized for control design were (i) LMED-1X to ACCEL-1X, (ii) LMED-1Y to ACCEL-1Y, (iii) LMED-2X to ACCEL-2X, and (iv) LMED-2Y to ACCEL-2Y. It was assumed that the open loop dynamics of each of the four loops was identical so that the same controller $\mathrm{H}(\mathrm{s})$ can be utilized in each loop. A block diagram of the assumed dynamics for each of the feedback loops is shown in Figure 4.3.1.

From Figure 4.3.1 it follows that the transfer function from the beam velocity (at the given LMED location and along a given axis) to the force applied (by the LMED along the same axis) is

$$
\frac{f_{c}}{\dot{x}_{p}}=\frac{H(s) s^{2}+D s+k_{m} k_{d}}{s^{2}+\frac{D}{m} s+\frac{k_{m} k_{d}}{m}} .
$$

The design goal was to choose $H(s)$ such that the above transfer function is positive real at low frequency (say around $1 \mathrm{~Hz}$ ) and remains positive real to some significantly higher frequency (say $10 \mathrm{~Hz}$ ) in order to provide damping to the beam modes in this frequency band. In theory the design objectives could be accomplished by simply choosing $H(s)=1 / s^{2}$. However, due to the stroke limitations of the LMED proof mass devices, this controller, which has very high gain at low frequency, is not implementable. Thus, $\mathrm{H}(\mathrm{s})$ was initially chosen to be a second-order low-pass filter. Unfortunately, even this controller caused the stroke limitations to be violated. To limit the low frequency stroke a first order high pass filter was then cascaded with the second order low pass 
filter. The resultant controller was thus of the form

$$
H(s)=\frac{k s}{(s+\alpha)\left(s^{2}+2 \zeta w_{n} s+w_{n}^{2}\right)} .
$$

The low and high pass portions of the controller were discretized separately by using the bilinear transformation with frequency prewarping and was then implemented in each of the loops. The control gains of the discretized controller are given in Appendix A.

The closed loop attenuation in the beam vibration is demonstrated in Figures 4.3.2.-4.3.7. Figure 4.3.2 shows the open and closed loop responses of BGYRO-Y to a BET-X pulse disturbance. It is seen that the LMED controllers especially aided in providing damping to the higher frequency harmonics. The closed loop performance improvement to a BET-X pulse is demonstrated even more clearly in Figures 4.3.3 and 4.3.4 which show the responses of ACCEL-1X and ACCEL-2X. The closed loop accelerometer responses reveal that both the peak magnitude of the responses and the influences of the higher frequency harmonics were significantly reduced. Similar results are seen if Figures 4.3.5-4.3.7 which show the BGYRO-Y, ACCEL-1Y and ACCEL-2Y responses to a BET-Y pulse disturbance. When integrated with the feedback controllers involving the IMC and AGS gimbals, the LMED loops improved the LOS performance especially by reducing the influence of the higher frequency vibration in the detector responses. 


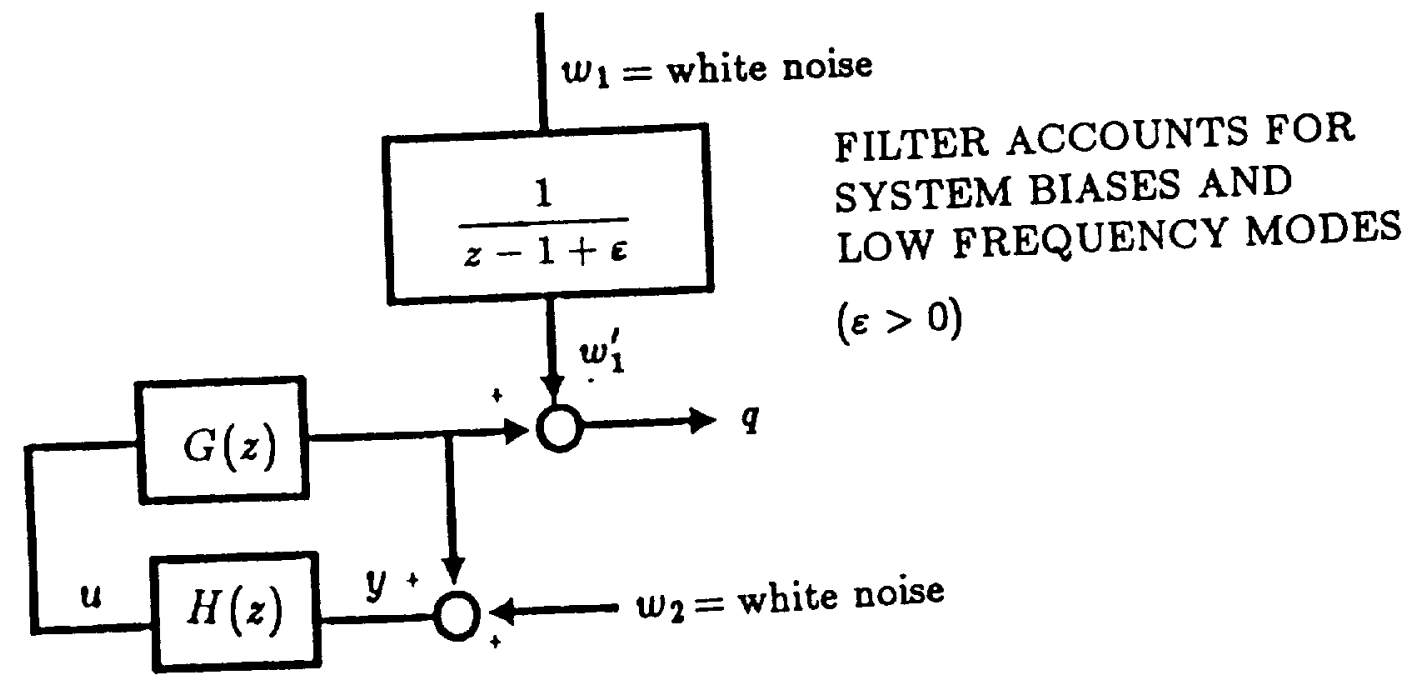

- CONTROLLERS WERE DESIGNED BY MINIMIZING

$$
J(\varepsilon)=\lim _{k \rightarrow \infty} E\left[q^{\mathrm{T}}(\varepsilon) q(\varepsilon)+\rho u^{\mathrm{T}}(\varepsilon) u(\varepsilon)\right], \quad \rho>0
$$

Figure 4.1.1 The control problem for both the IMC-X to DET-Y loop and the IMC-Y to DET-X loop was formulated as a disturbance tracking problem. 

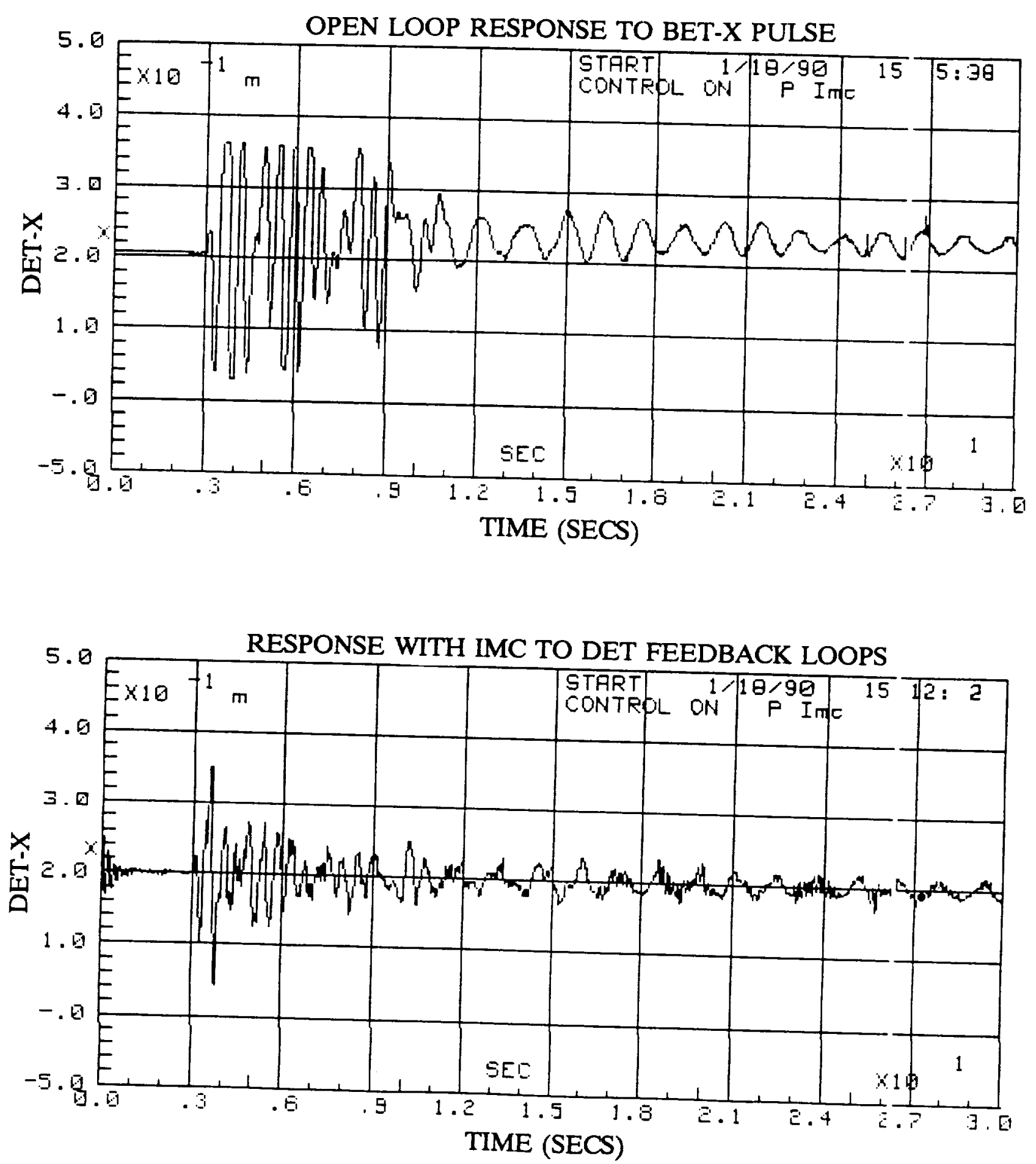

Figure 4.1.2. The IMC to DET feedback loops were able to provide low frequency tracking in addition to bias correction to improve the DET-X response to a BET-X pulse disturbance. 

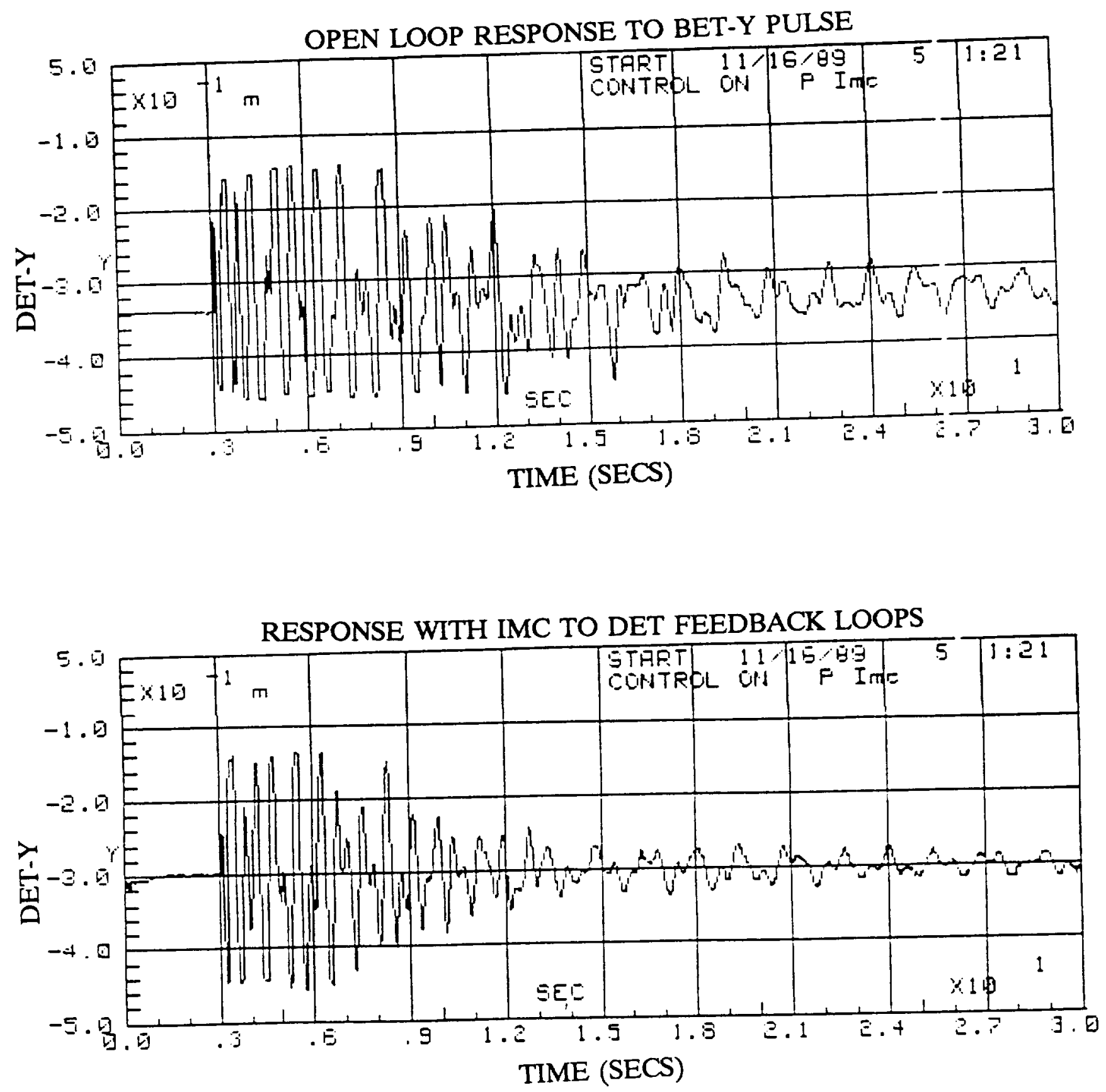

Figure 4.1.3. The IMC to DET feedback loops were able to provide low frequency tracking in addition to bias correction to substantially improve the DET-Y response to a BET-Y pulse disturbance. 


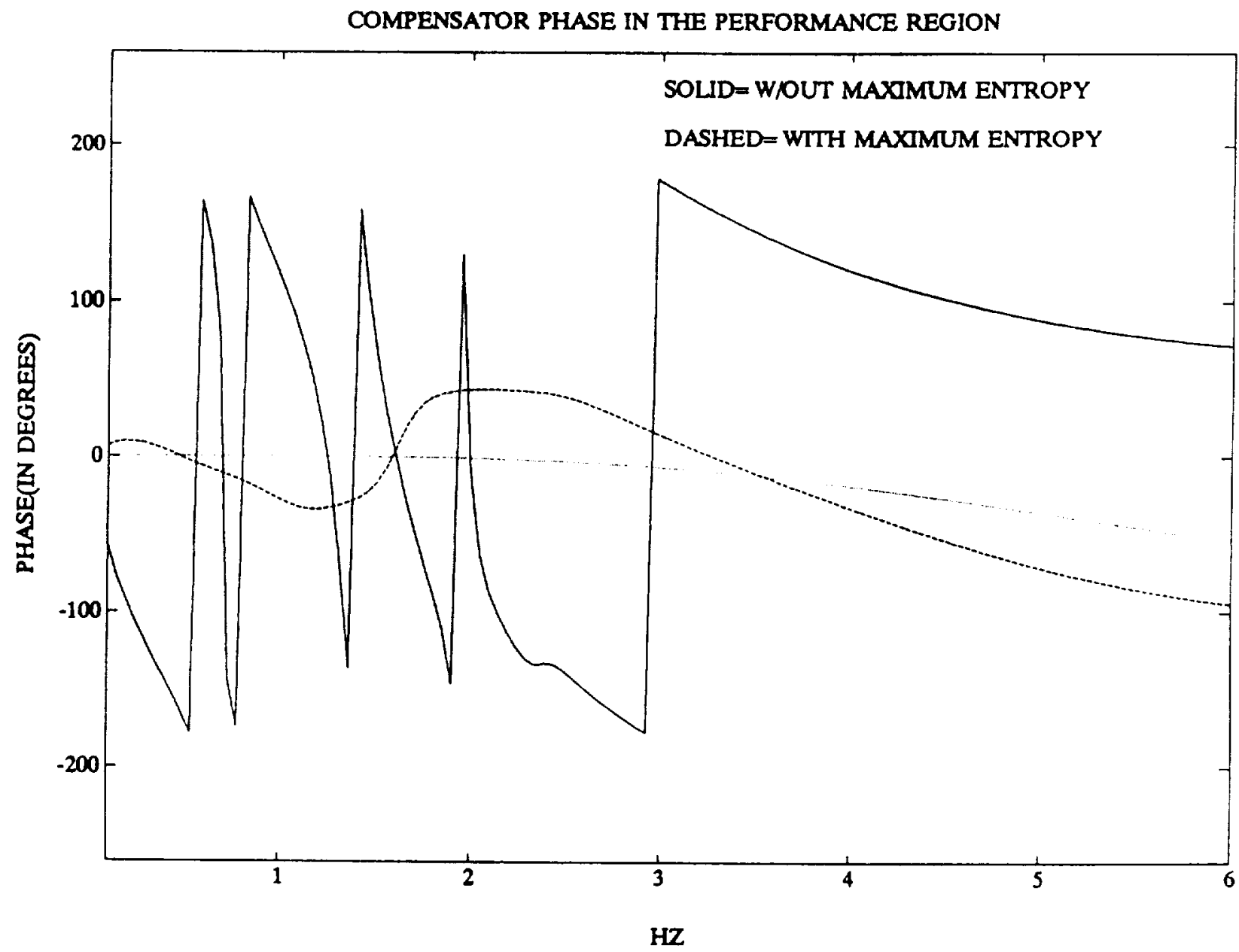

Figure 4.2.1. Maximum Entropy design rendered the compensators for the AGS to BGYRO loops positive real in the performance region. 


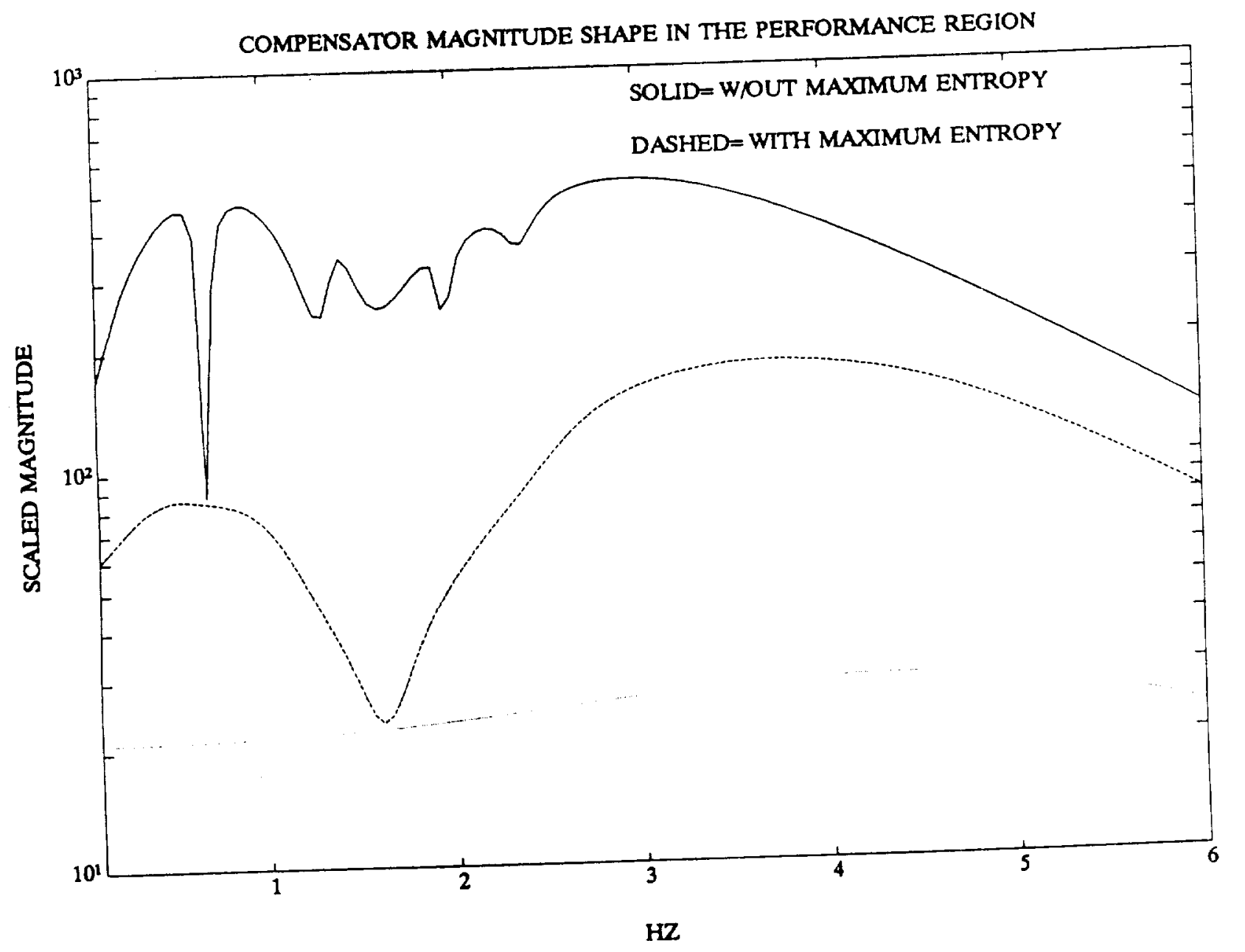

4.2.2. For the AGS to BGYRO loops Maximum Entropy design smoothed out the compensator magnitudes in the performance region, thus providing performance robustness and also indicating that the robust controllers were effectively reduced-order controllers. 


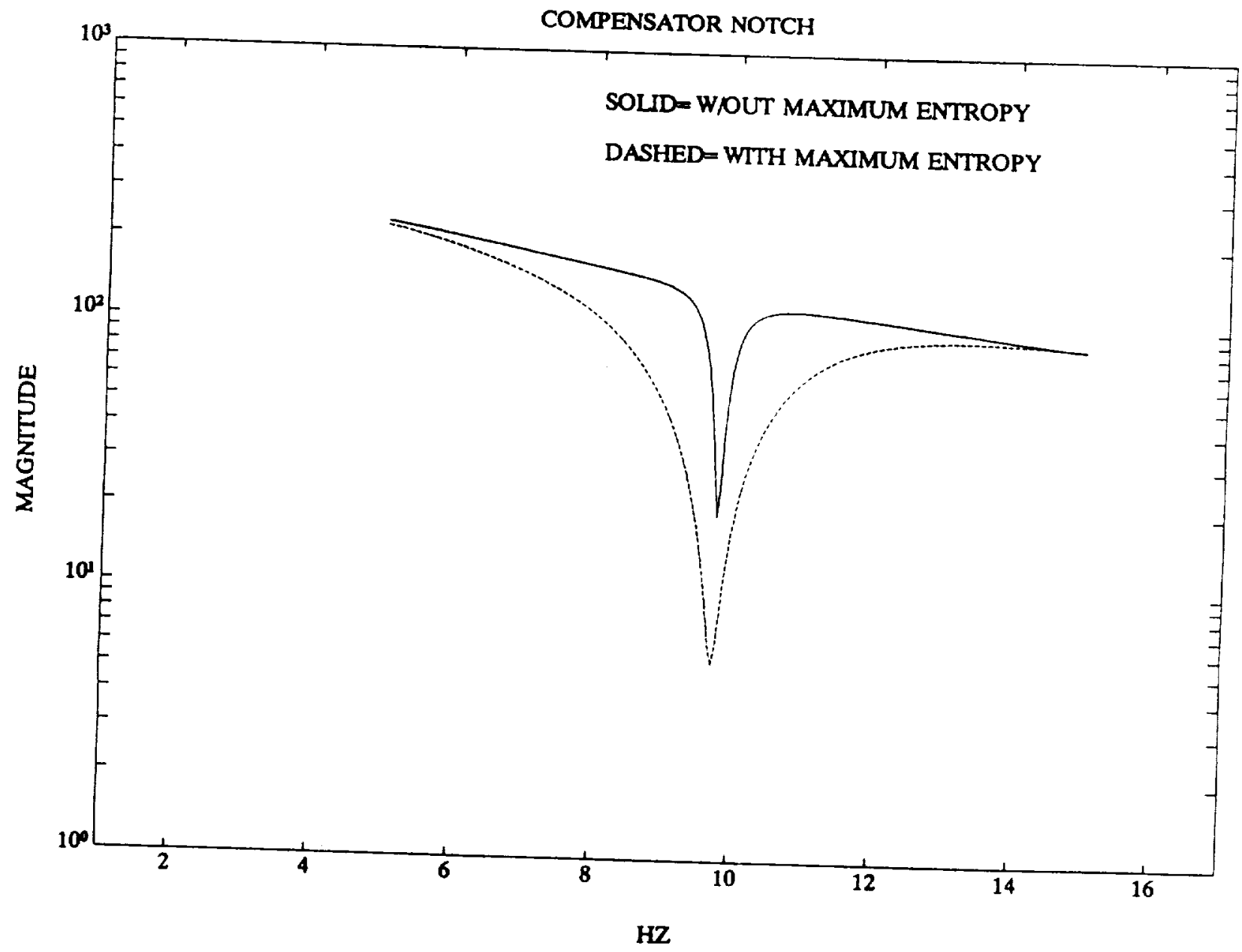

4.2.3. For the AGS to BGYRO loops Maximum Entropy design robustified the notches for the high frequency modes by increasing their width and depth. 

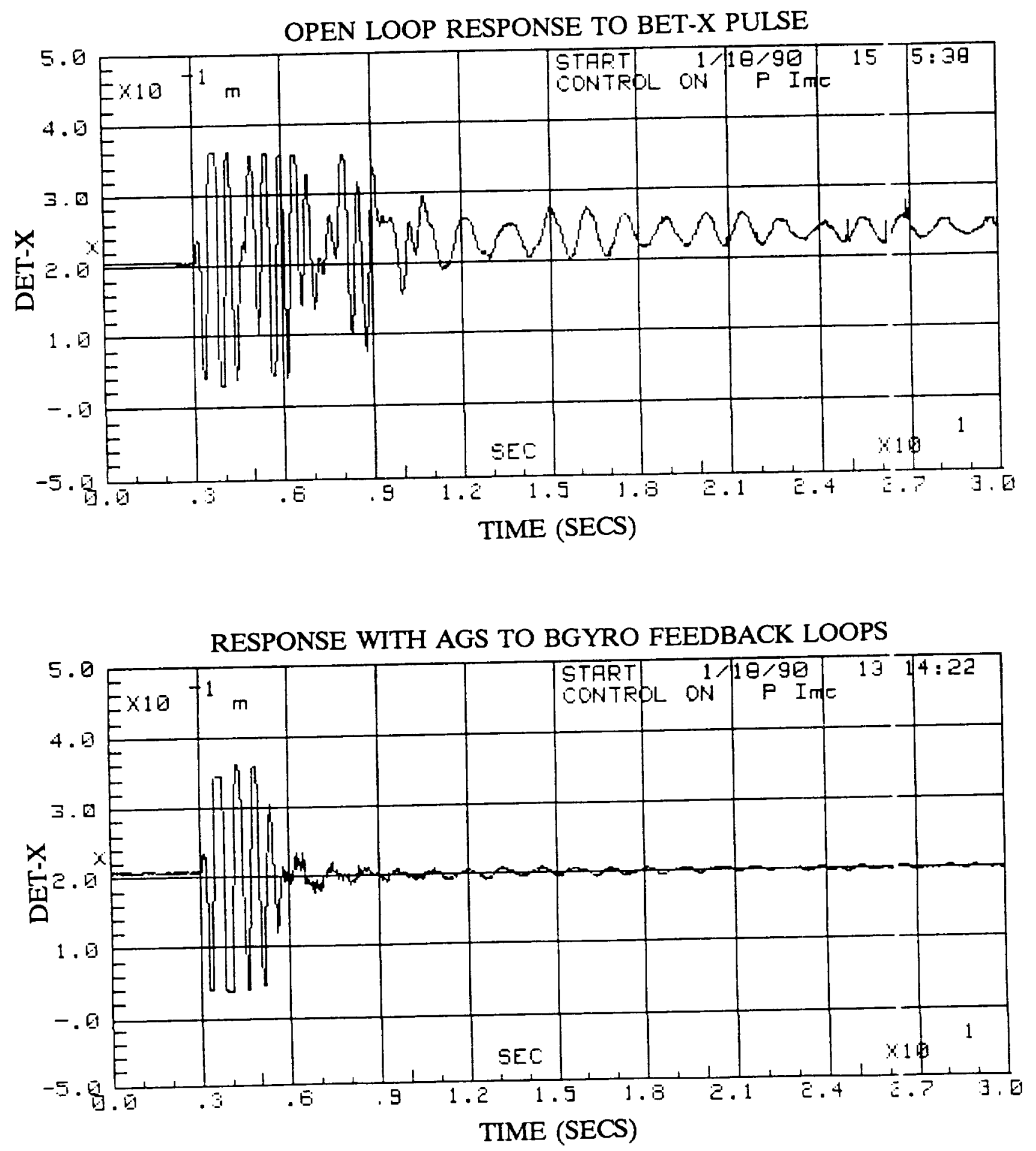

Figure 4.2.4. The reduction beam vibration of the AGS to BGYRO feedback loops was able to substantially improve the DET-X response to a BET-X pulse disturbance. 

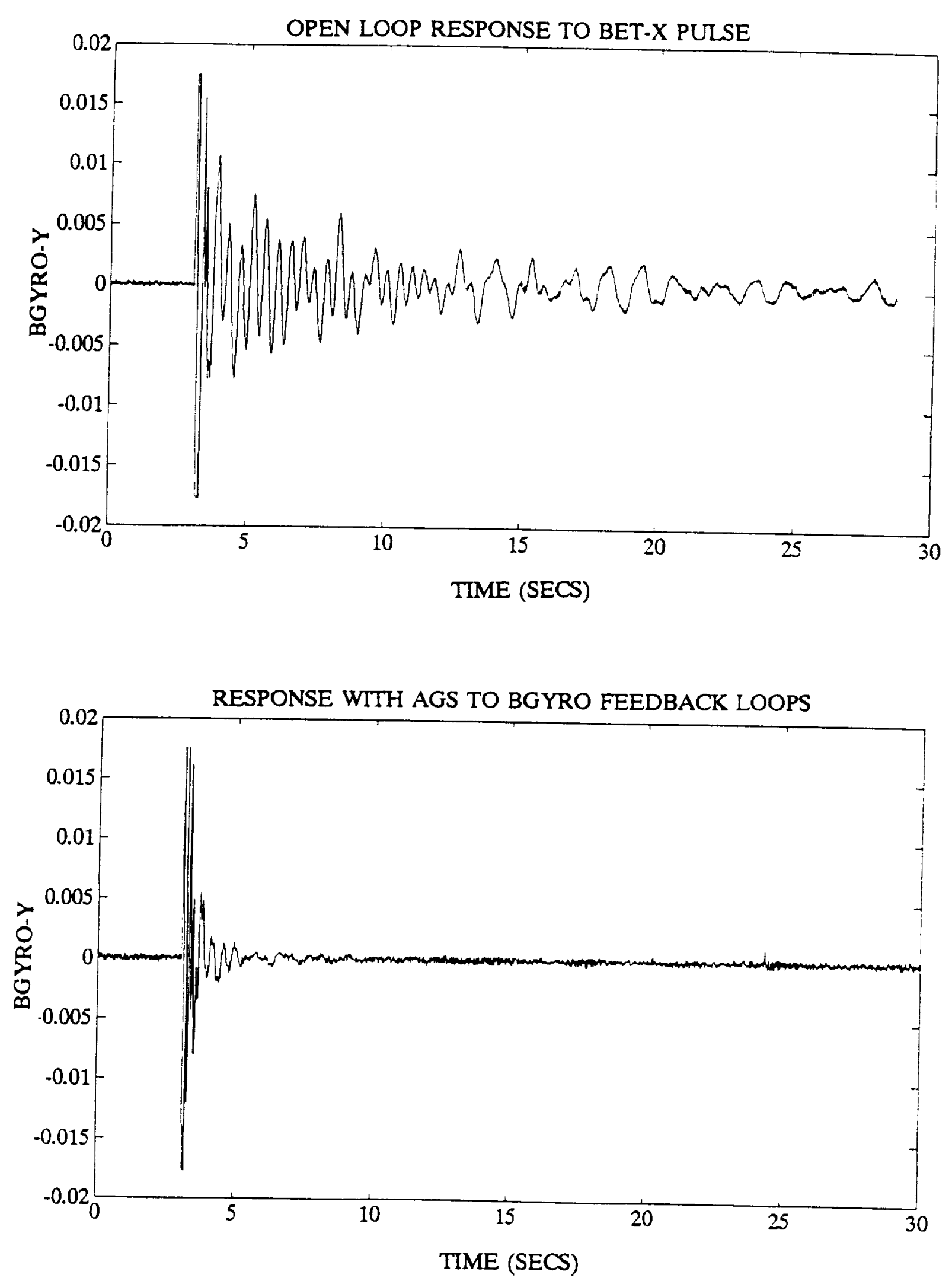

Figure 4.2.5. For a BET-X pulse a comparison of the open loop BGYRO-Y response to the response with the AGS to BGYRO feedback loops closed reveals significant closed loop damping
of the beam vibration. 

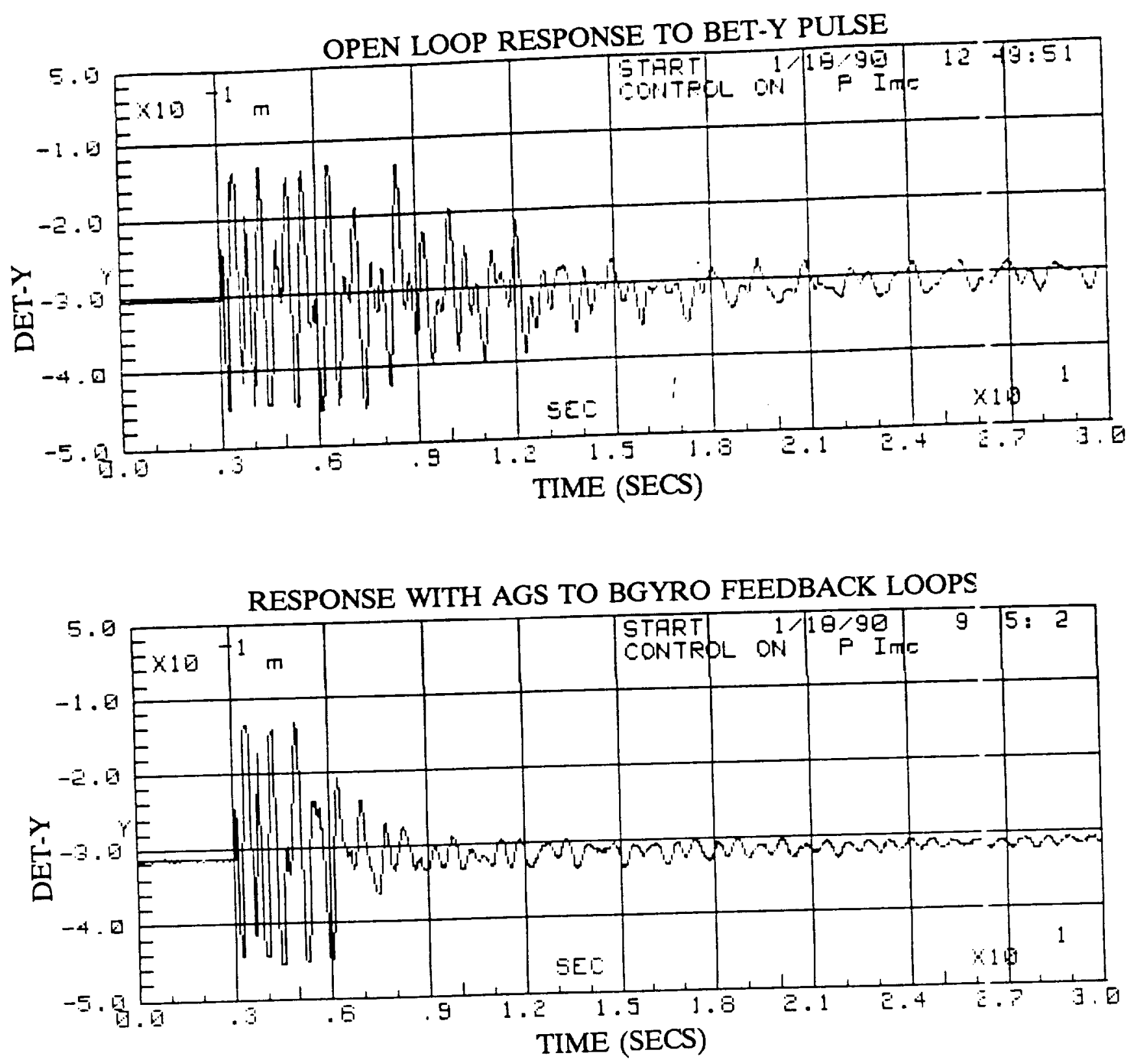

Figure 4.2.6. The reduction in beam vibration of the AGS to BGYRO feedback loops was able to substantially improve the DET-Y response to a BET-Y pulse disturbance. 

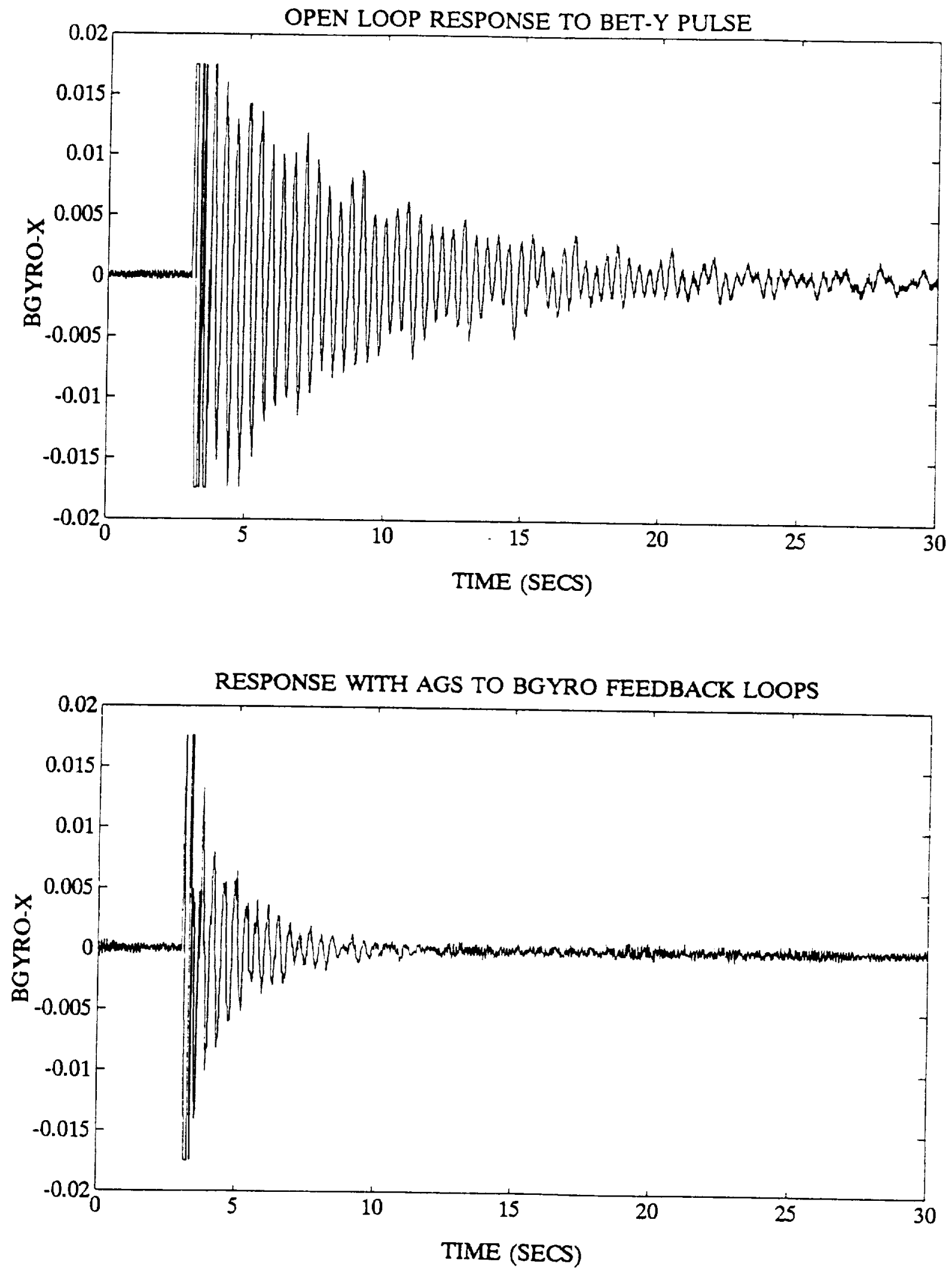

Figure 4.2.7. For a BET-Y pulse a comparison of the open loop BGYRO-X response to the response with the AGS to BGYRO feedback loops closed reveals significant closed loop damping of the beam vibration. 


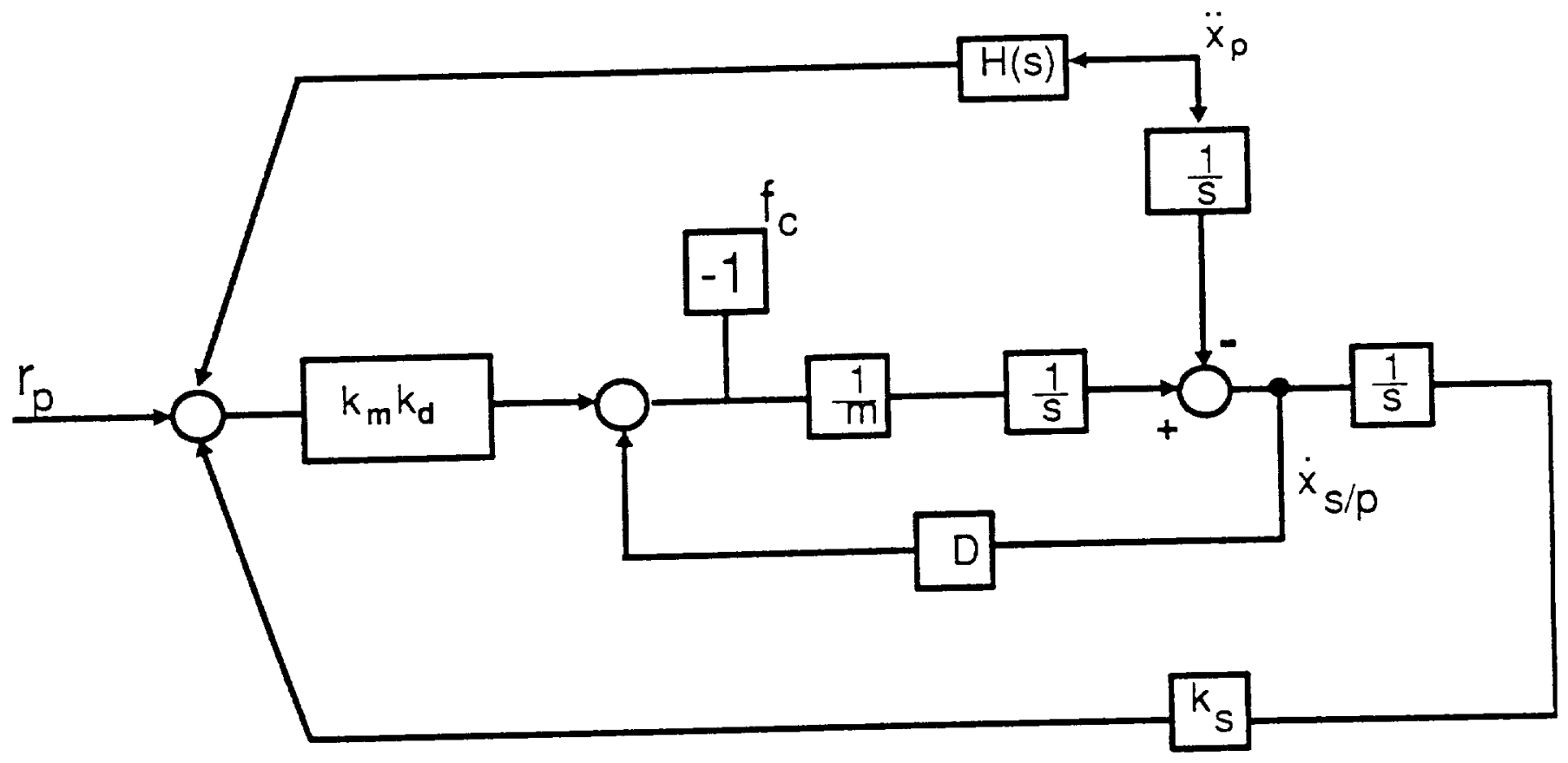

$\ddot{x}_{p}=$ beam acceleration

$\dot{x}_{s / p}=$ relative velocity of proof mass

$\mathrm{m}=$ mass of proof mass

$f_{C}=$ force applied to structure

$k_{S}=$ position loop stiffness

$D=$ inherent viscous damping of the LMED

$k_{m} k_{d}=$ motor force constants

$H(s)=$ compensator transfer function

$r_{p}=$ position command.

Figure 4.3.1. The LMED designs assumed that this block diagram described each feedback loop with control law $\mathrm{H}(\mathrm{s})$. 

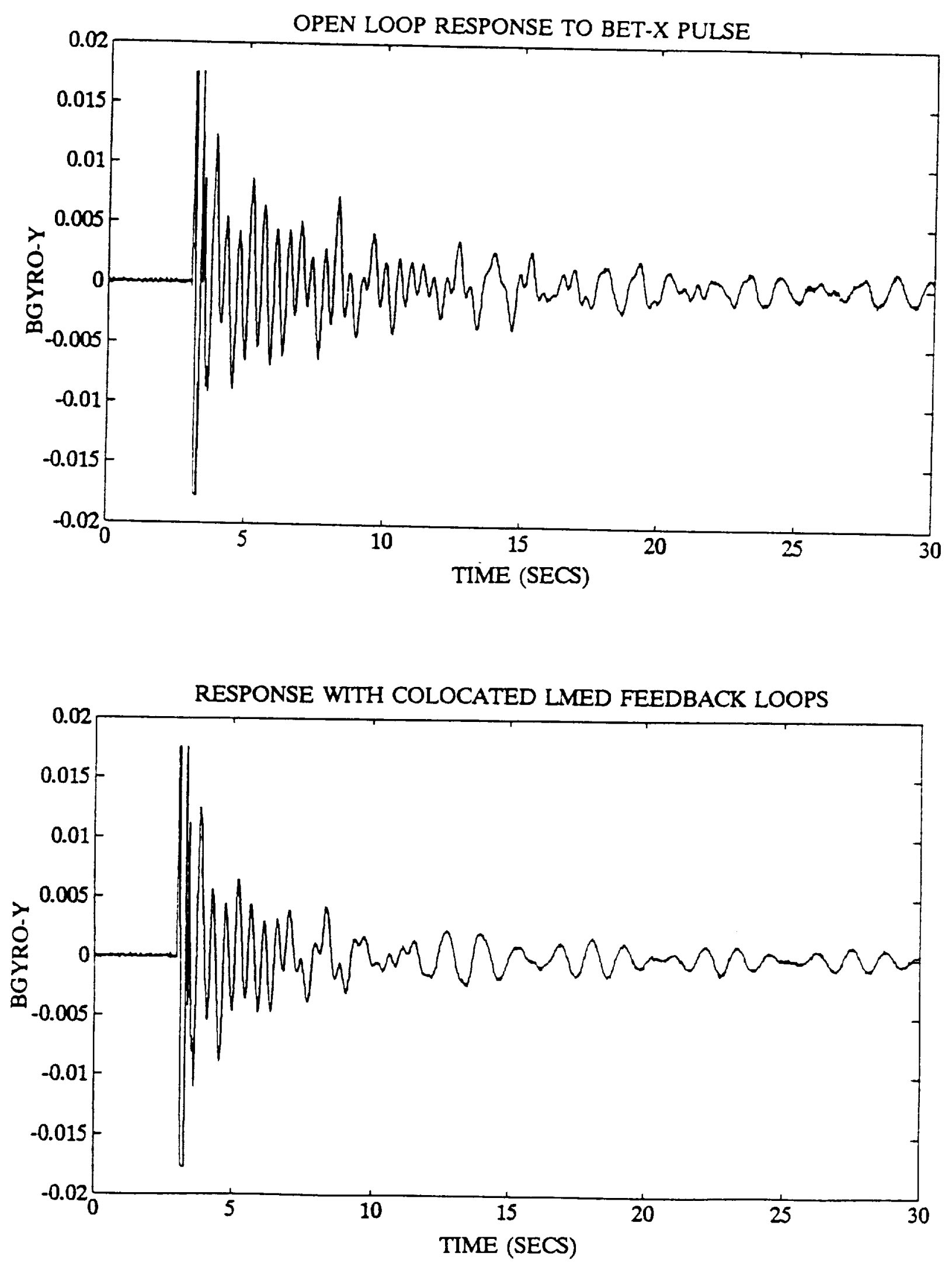

Figure 4.3.2. For a BET-X pulse a comparison of the open loop BGYRO-Y response to the response with the LMED loops closed reveals some closed loop damping of the higher frequency harmonics. 

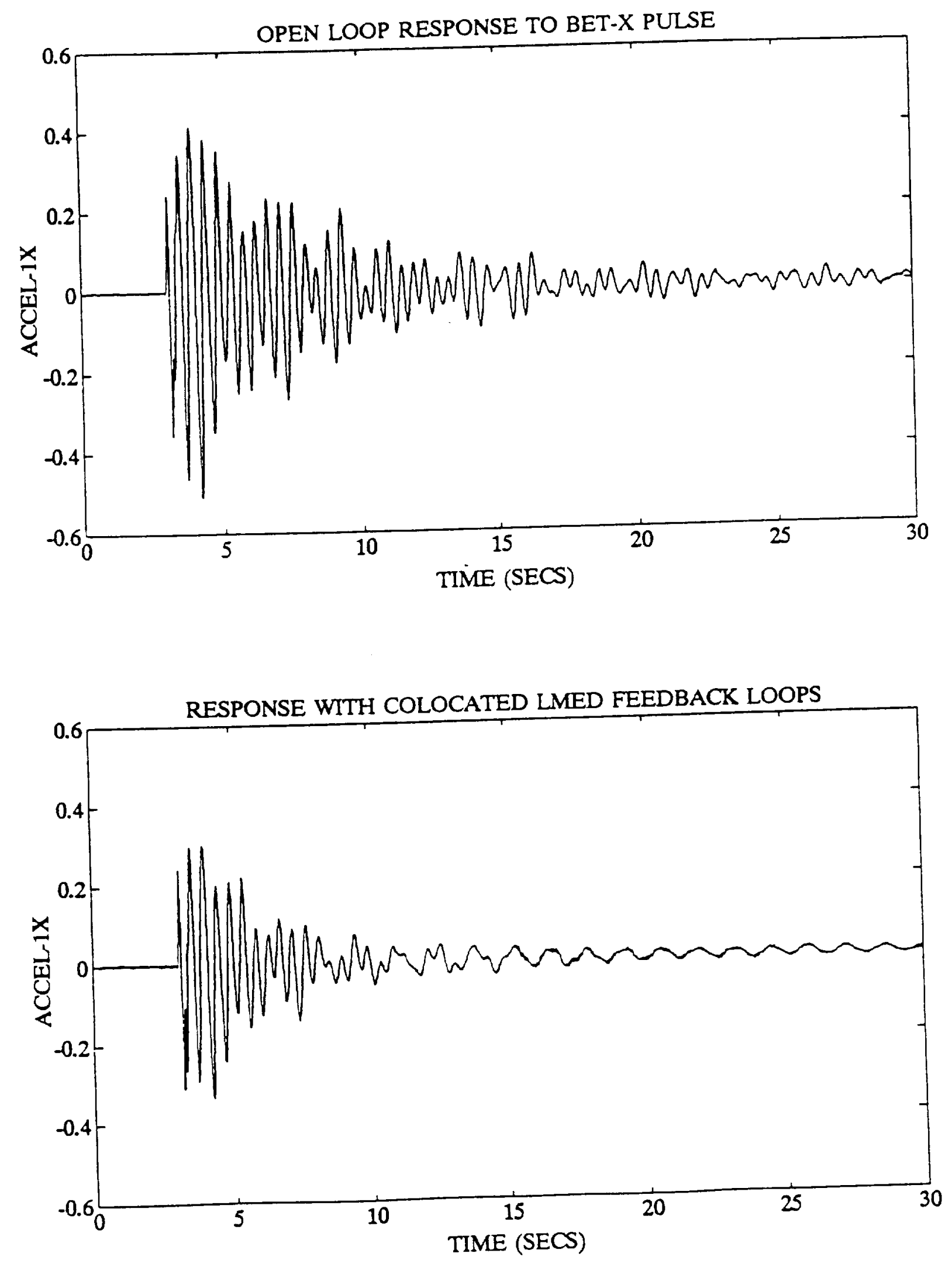

Figure 4.3.3. For a BET-X pulse a comparison of the open loop ACCEL-1X response to the response with the LMED loops closed reveals some closed loop damping of the higher frequency harmonics. 

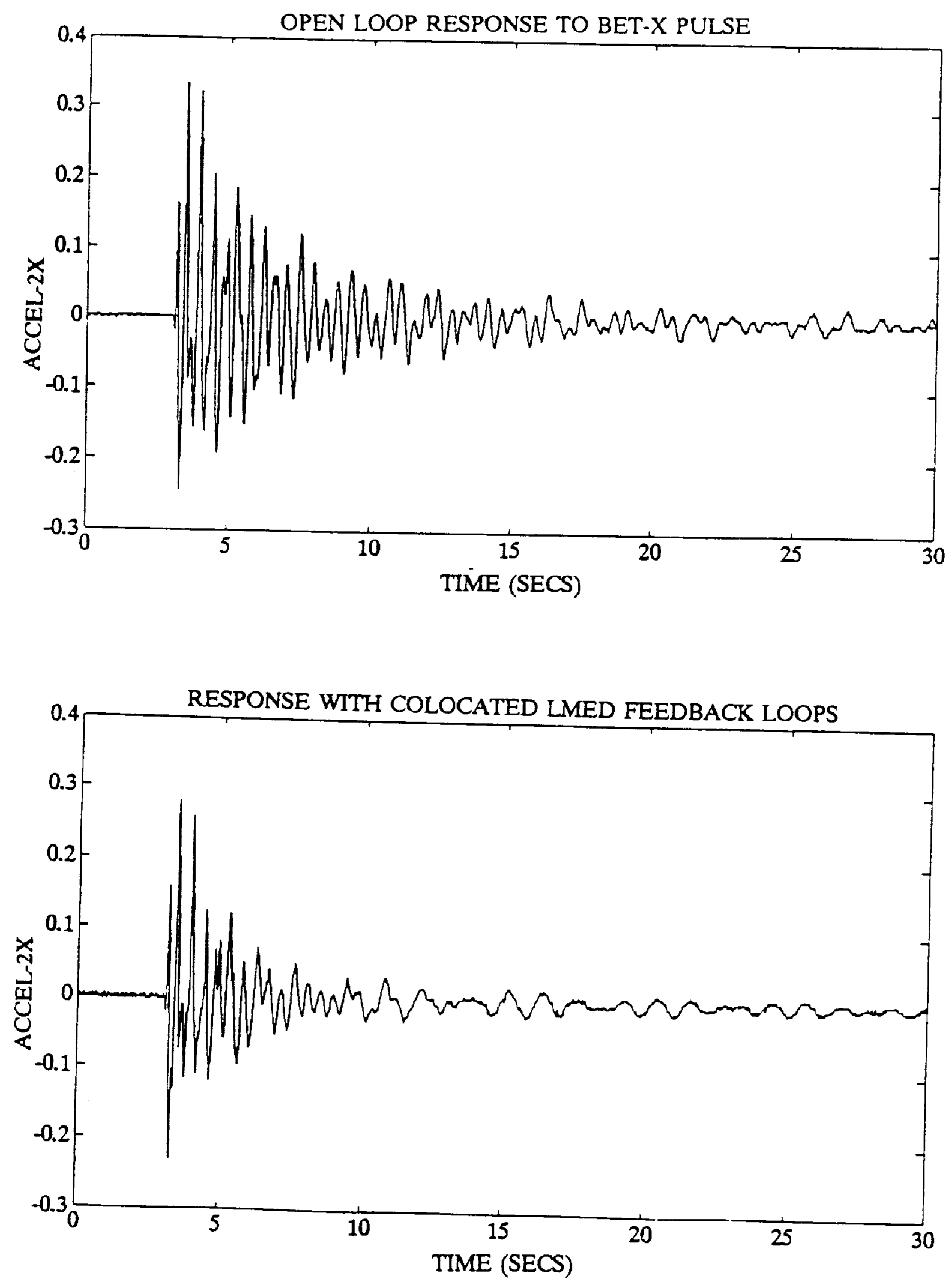

Figure 4.3.4. For a BET-X pulse a comparison of the open loop ACCEL-2X response to the response with the LMED loops closed reveals some closed loop damping of the higher frequency
harmonics. 

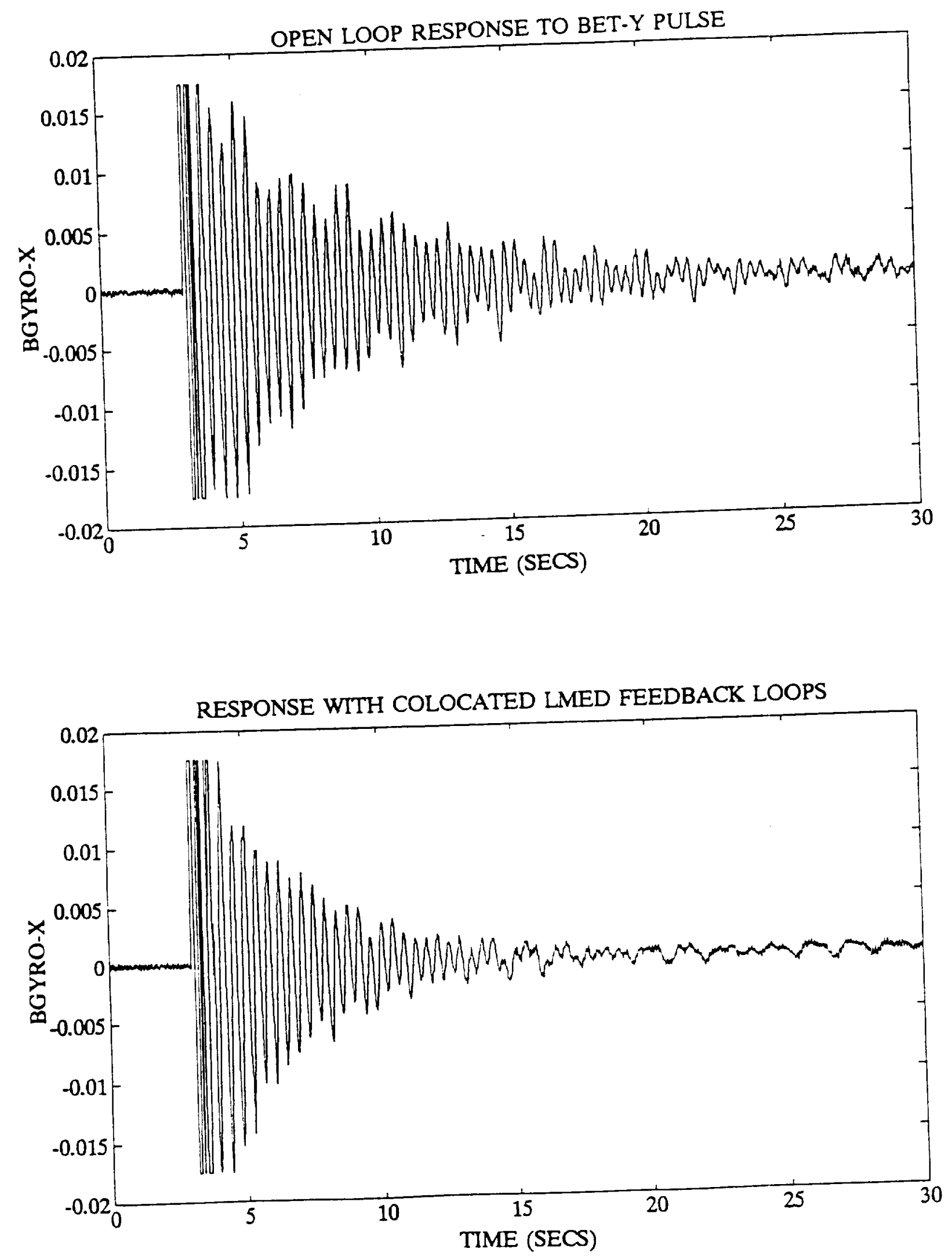

Figure 4.3.5. For a BET-Y pulse a comparison of the open loop BGYRO-X response to the response with the LMED loops closed reveals some closed loop damping of the higher frequency harmonics. 

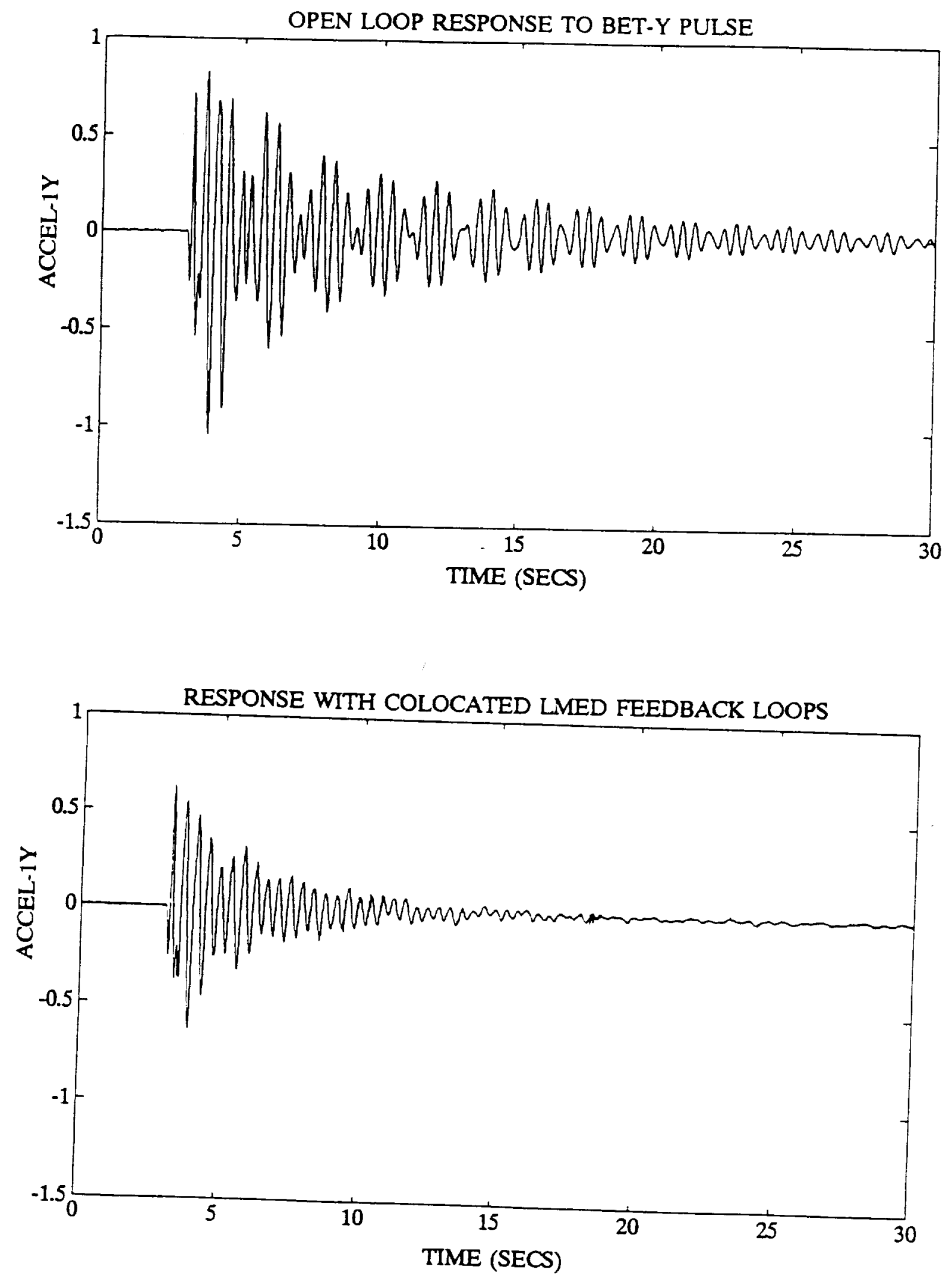

Figure 4.3.6. For a BET-Y pulse a comparison of the open loop ACCEL-1Y response to the ACCEL-1Y response with the LMED loops closed reveals some closed loop damping of the higher
frequency harmonics. 

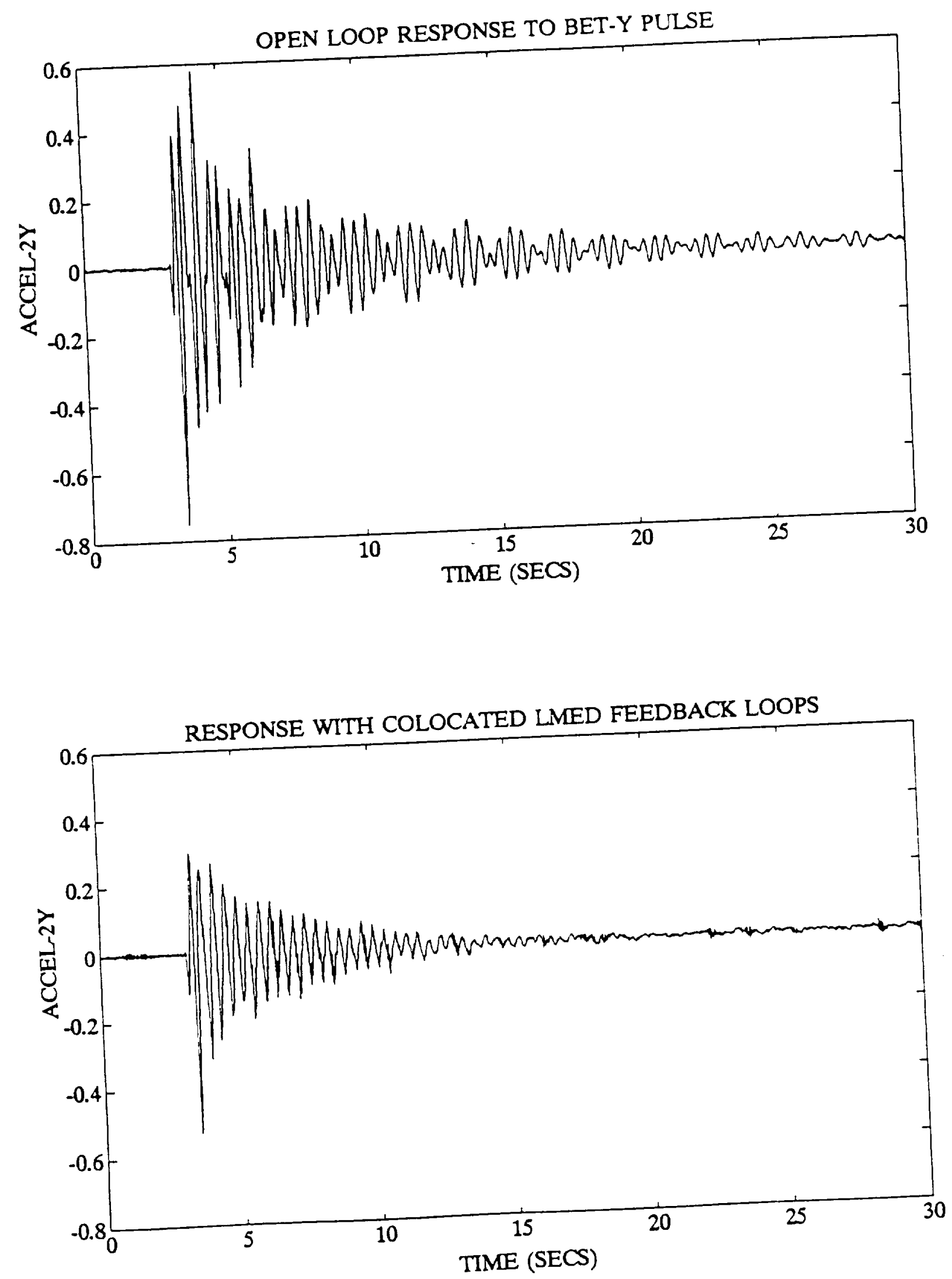

Figure 4.3.7. For a BET-Y pulse a comparison of the open loop ACCEL-2Y response to the ACCEL-2Y response with the LMED loops closed reveals some closed loop damping of the higher frequency harmonics. 
工 


\section{PERFORMANCE OF THE INTEGRATED CONTROLLER}

The integrated controller consisted of decentralized controllers for each of the three major feedback paths. Since the order of the controllers for the IMC gimbal to detector loops, the AGS gimbal to base gyro loops, and the colocated LMED loops were respectively 6th, 10th and 12th order, the integrated controller consisted of 28 states. The controller was evaluated for three types of disturbance commands to the BET-X and BET-Y: (i) pulse disturbances, (ii) the RCS disturbance described by Figure 5.2.1, and (iii) the Crew disturbance described by Figure 5.3.1.

The two measure of performance that were computed to compare the open-loop and closed-loop performance are the mean $(\bar{q})$ and the standard deviation $(\sigma)$, defined respectively as $[7$, p. $2-24]$

$$
\begin{aligned}
& \bar{q} \triangleq \frac{1}{N} \sum_{i=1}^{N} q_{i} \\
& \sigma \triangleq \sqrt{\left(\sum_{i=1}^{N} q_{i}^{2}-N \bar{q}^{2}\right) /(N-1)}
\end{aligned}
$$

where for a given sensor output $N$ is the number of recorded samples over a specified time interval and $\left\{q_{i}\right\}_{i=1}^{N}$ is the sequence of sampled values. The performance improvement in $\mathrm{dB}$ for both the mean and standard deviation is defined as

$$
\text { Performance Improvement }=20 \log _{10}\left(\frac{\text { OL performance }}{\text { CL performance }}\right) \text {. }
$$

\subsection{Response Due To Pulse Disturbances}

For pulse commands to BET-X and BET-Y Tables 5.1 and 5.2 show the resultant performance improvement respectively in the DET-X and DET-Y responses as the controllers were integrated. In terms of the standard deviation, the integrated controller yielded a $9.0 \mathrm{~dB}$ improvement in the DET-X response and a $2.4 \mathrm{~dB}$ improvement in the DET-Y response. The corresponding improvements in the mean were respectively $57.9 \mathrm{~dB}$ and $47.5 \mathrm{~dB}$. These large values are due to large open-loop detector biases which were effectively eliminated using feedback. In general, the mean values were dominated by the size of the initial biases which varied with each test.

Figures 5.1.3-5.1.6 show the DET-X, BGYRO-Y, ACCEL-1X and ACCEL-2X responses to a BET-X pulse disturbance. A comparison of the open-loop and closed-loop responses shows very significant improvement in both the LOS errors and vibration suppression. For a BET-Y pulse 
disturbance similar performance improvement is seen in Figures 5.1.7-5.1.10 which show the DETY, BGYRO-X, ACCEL-1Y and ACCEL-2Y responses. By comparing Figures 5.1.3-5.1.10 with the corresponding figures of Section 4 it is easy to see that the integrated controller always yielded better performance than any of the three individual feedback controllers.

A comparison of Figures 5.1.3 and 5.1.7 shows that the performance improvement in DET-Y, though significant, is not as substantial as the improvement in DET-X. We conjecture that this is due to the interaction between the IMC-X gimbal, which most influences the DET-Y response, with the modes of the arm on which the IMC gimbal is mounted.

\subsection{Response Due to an RCS Disturbance}

The RCS disturbance profile is shown in Figure 5.2.1. Figures 5.2.2 and 5.2.3 show the DET-X and BGYRO-Y responses to an RCS disturbance command to BET-X. The controller improved the standard deviation of the DET-X response by $9.7 \mathrm{~dB}$ and the standard deviation of the BGYRO-Y response by $3.0 \mathrm{~dB}$.

\subsection{Response Due to a Crew Disturbance}

The Crew Disturbance profile is shown in Figure 5.3.1. Figures 5.3.2 shows the DET-X response to a Crew disturbance command to BET-X. The resultant improvement in the standard deviation was $4.3 \mathrm{~dB}$.

\subsection{Some Final Remarks on the Implementation Results}

The closed-loop test data indicated that sensor noise was not a significant factor in limiting performance. This was primarily due to the quality of the sensors and the dominance of other factors limiting the achievable performance (e.g., modeling errors and sampled-data issues). Also, Actuator saturation did not occur in either the AGS or IMC gimbals. However, we believe that actuator saturation will be an important factor when designing higher performance controllers involving the LMED's. In particular, care must be taken in designing the controllers so that the mass positions of these proof-mass devices do not try to exceed the physical limits. 


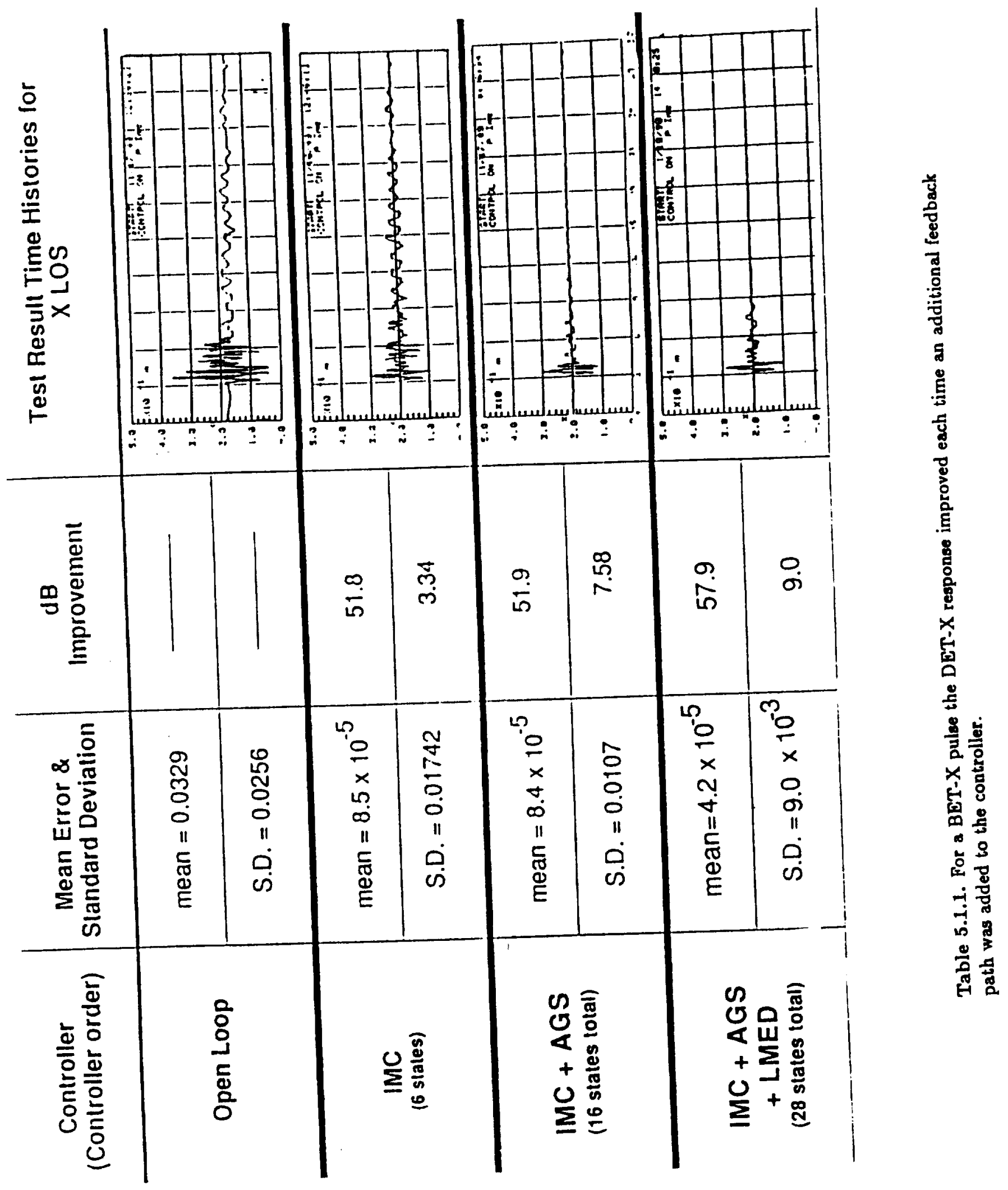




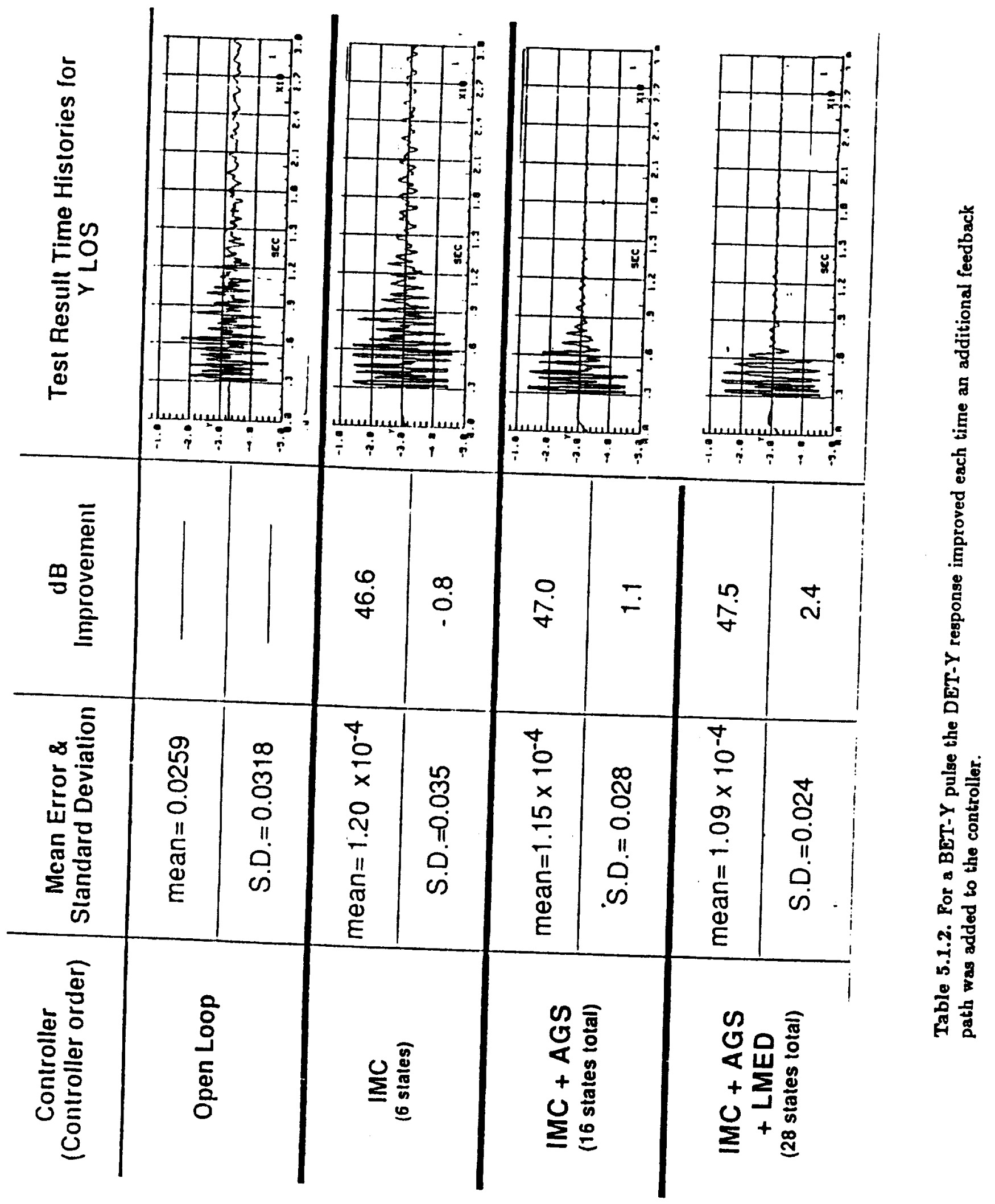



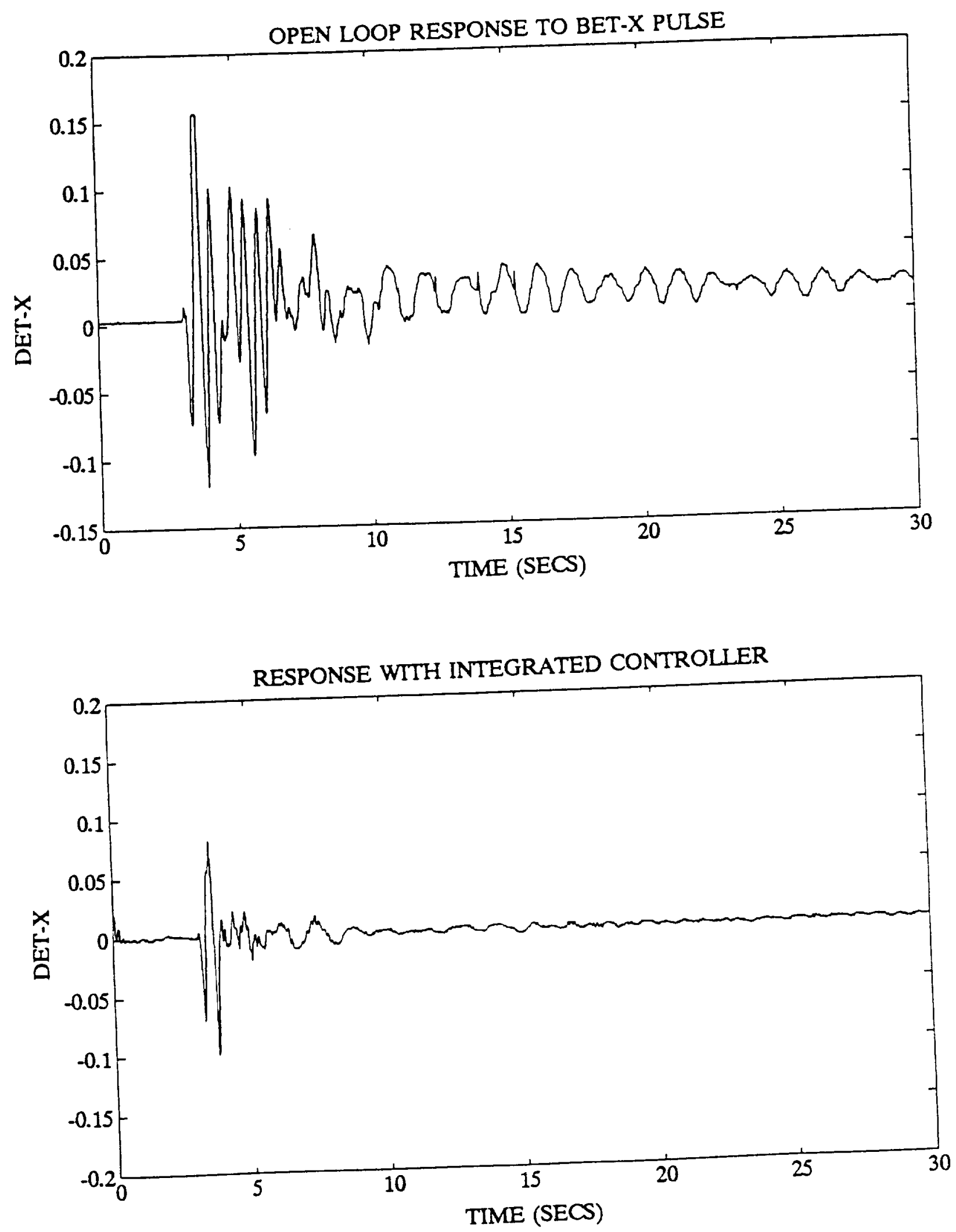

Figure 5.1.3. The integrated controller provided greater improvement in the DET-X response to a BET-X pulse than any of the three individual controllers. 

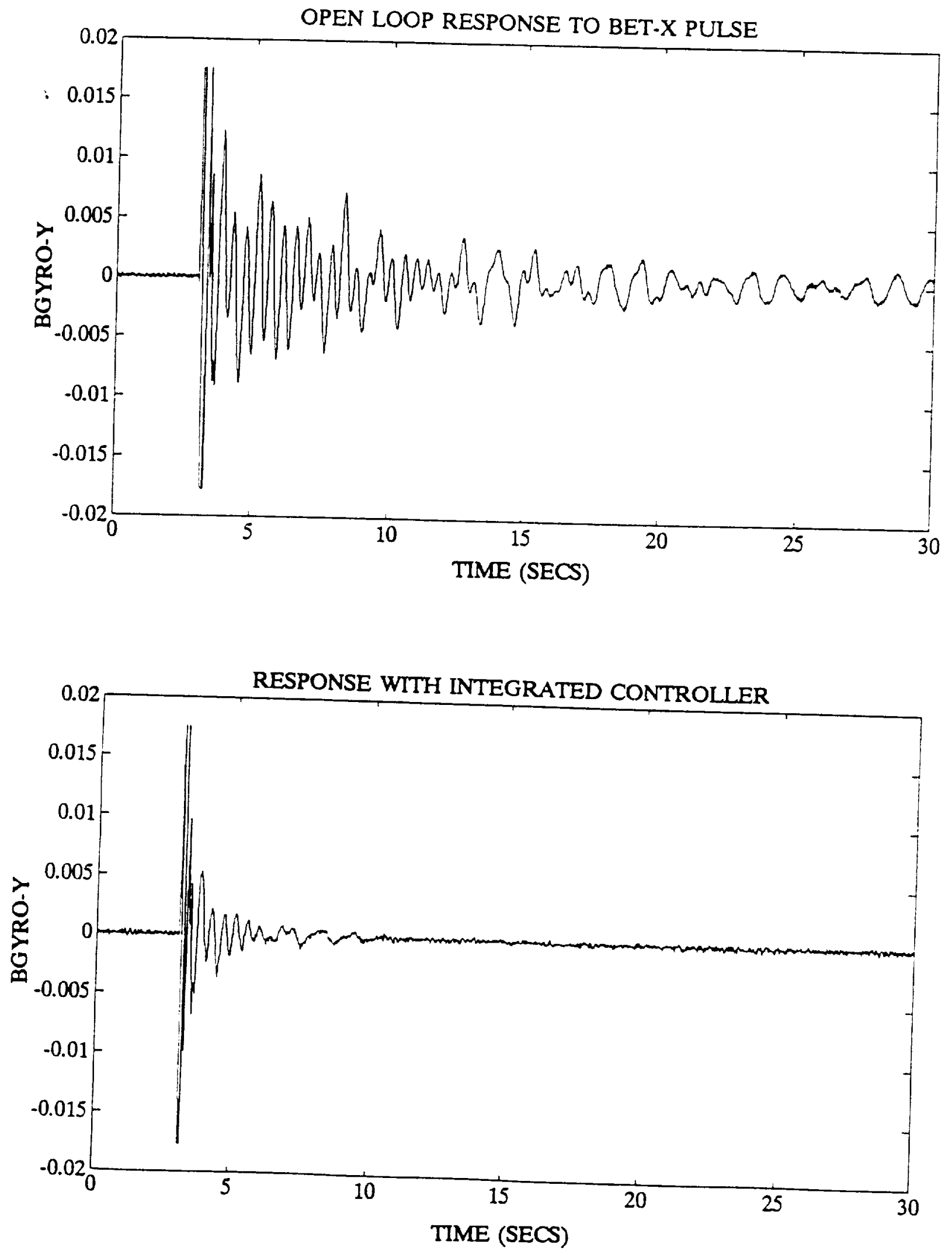

Figure 5.1.4. The integrated controller provided greater improvement in the beam damping as measured by the BGYRO-Y response to a BET-X pulse than any of the three individual controllers. 

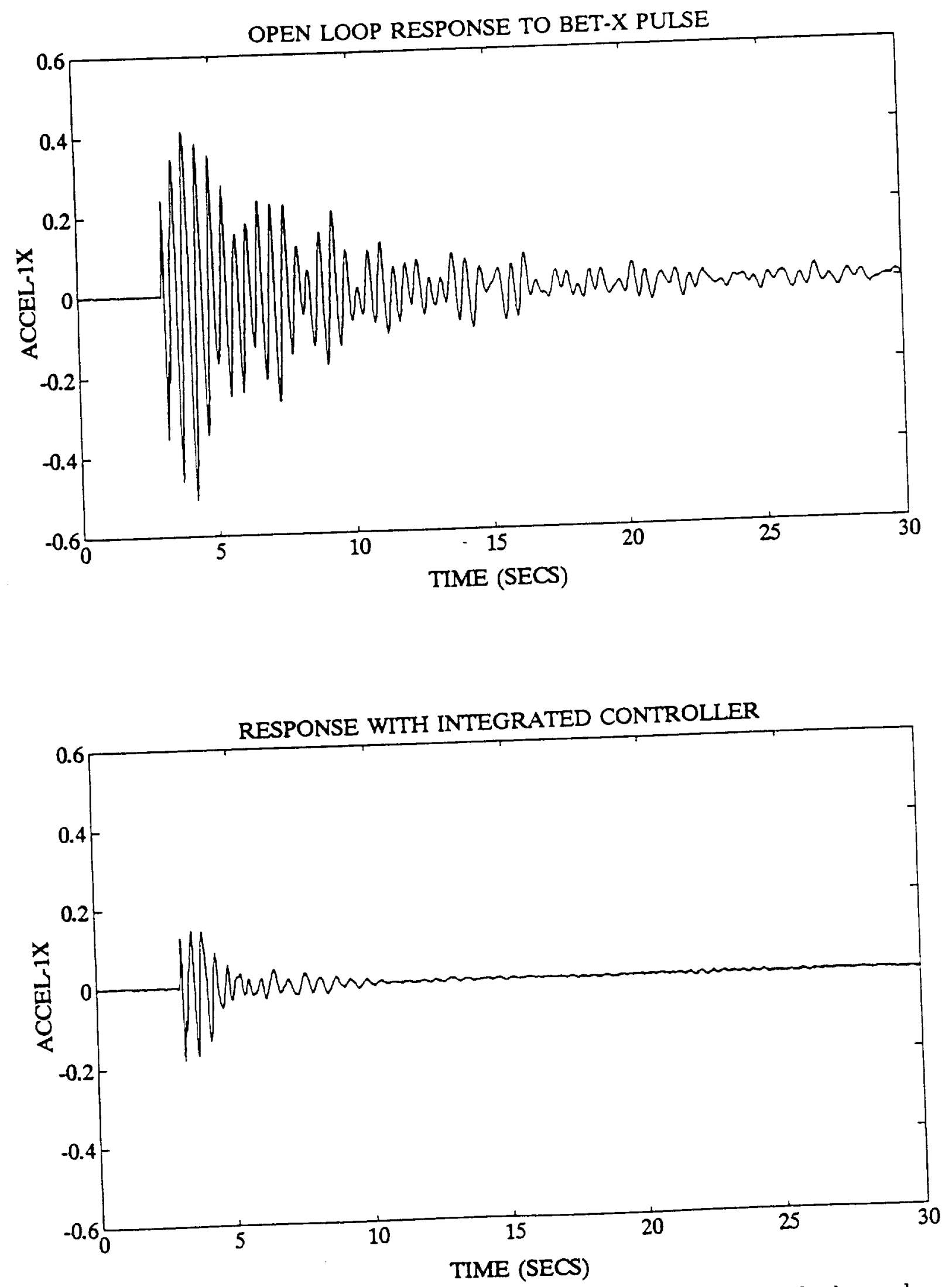

Figure 5.1.5. The integrated controller provided greater improvement in the beam damping as measured by the ACCEL-1X response to a BET-X pulse than any of the three individual controllers. 

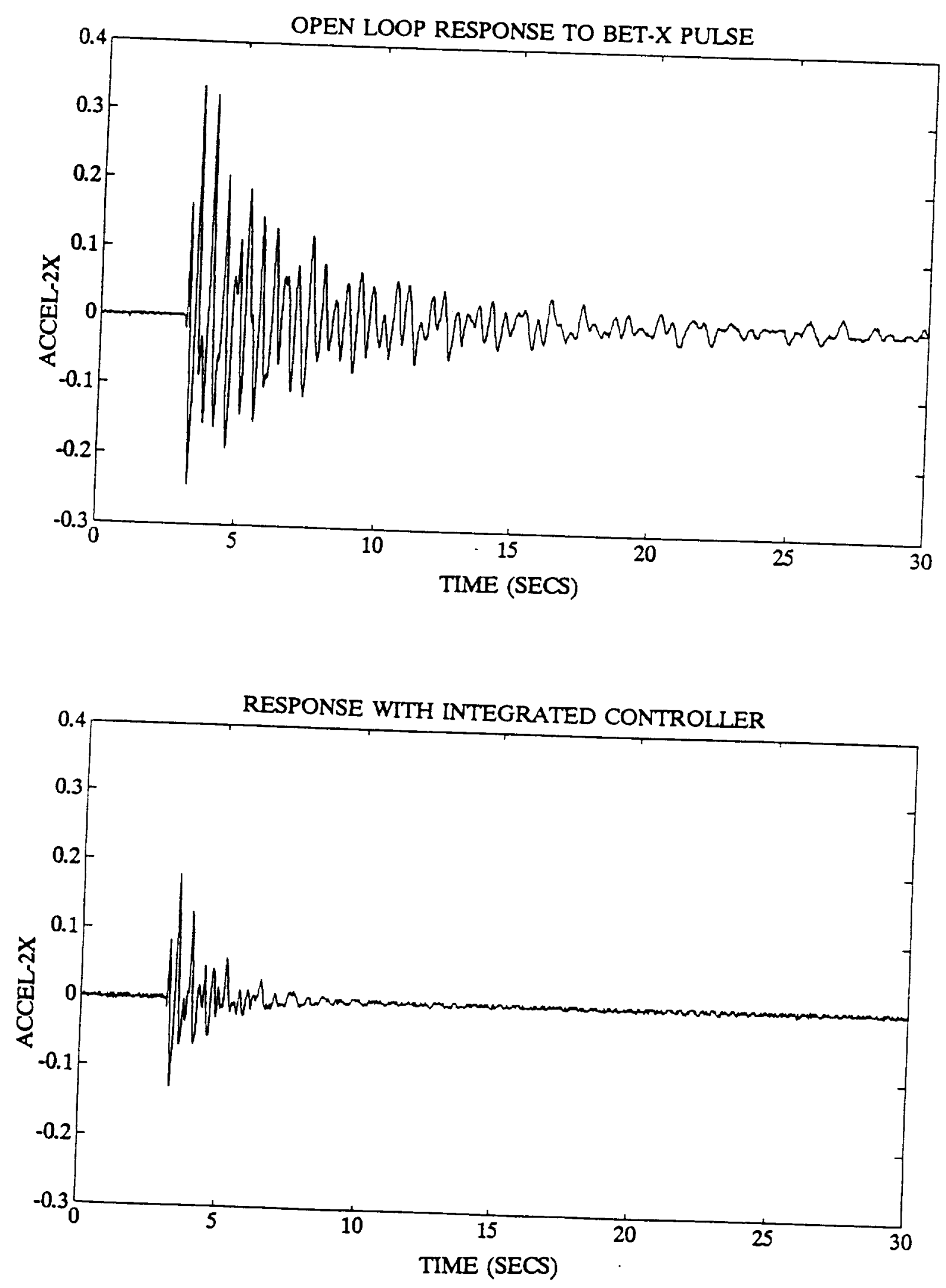

Figure 5.1.6. The integrated controller provided greater improvement in the beam damping as measured by the ACCEL-2X response to a BET-X pulse than any of the three : individual
controllers. 

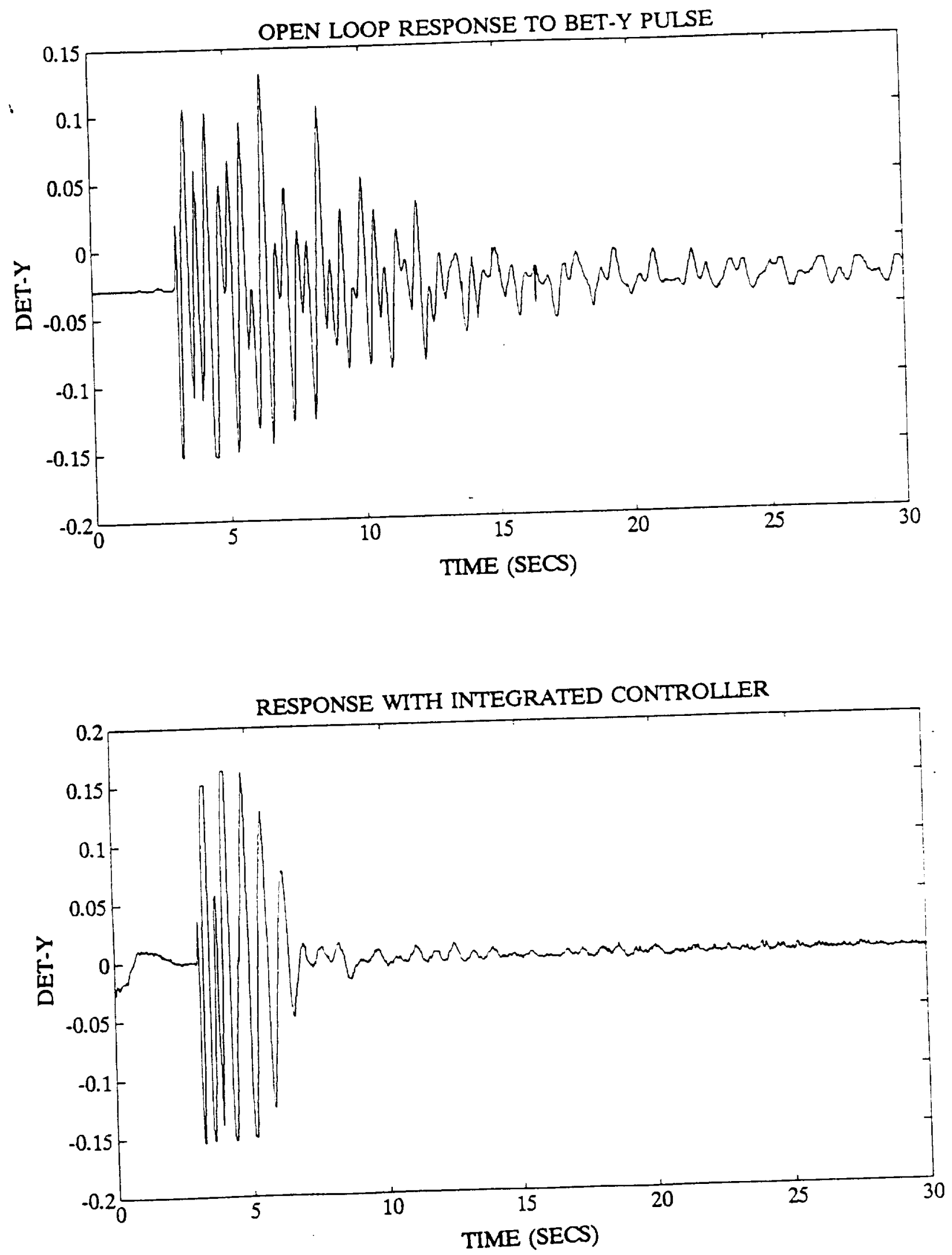

Figure 5.1.7. The integrated controller provided greater improvement in the DET-Y response to BET-Y pulse than any of the three individual controllers. 

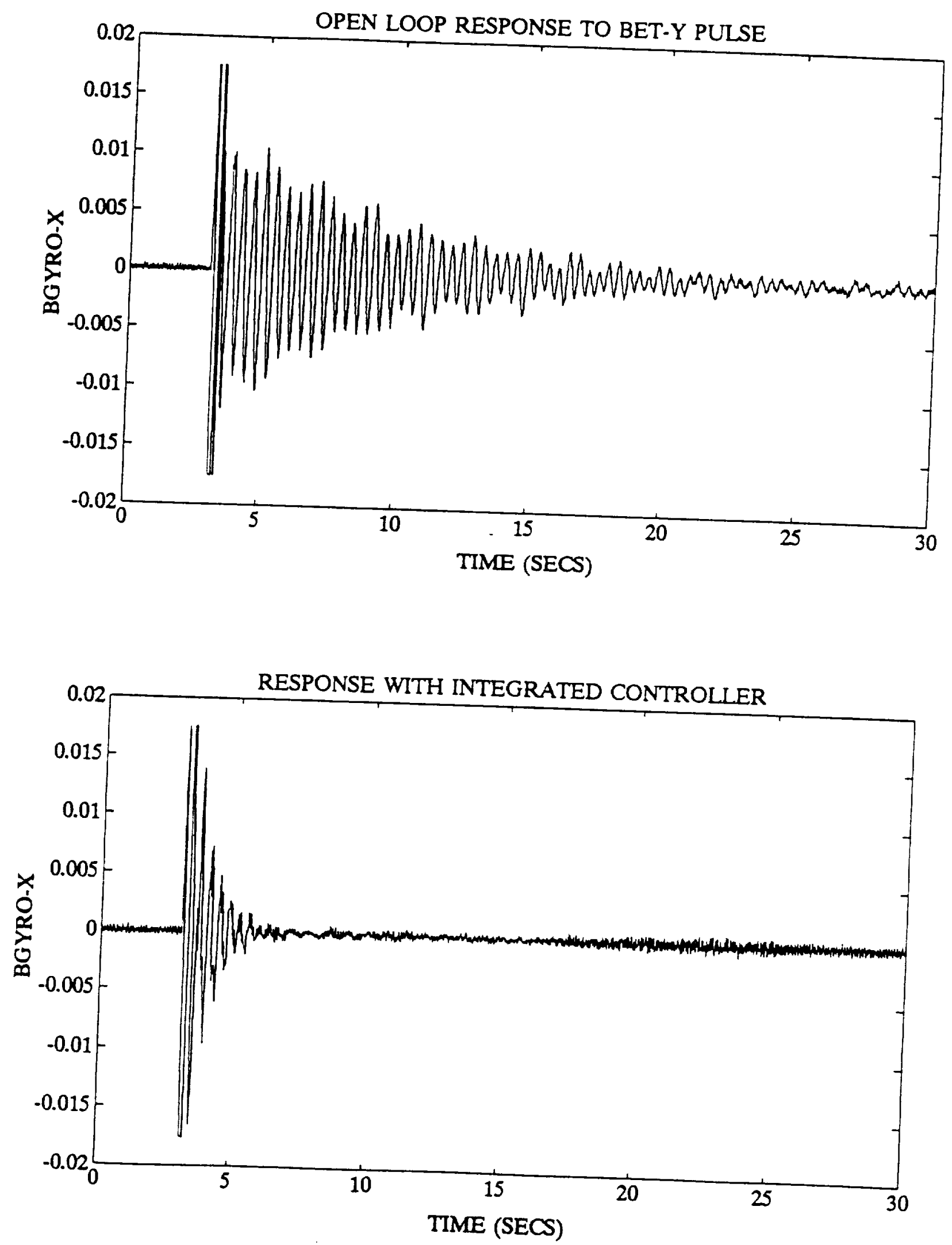

Figure 5.1.8. The integrated controller provided greater improvement in the beam damping as
measured by the BGYRO-X response to a BET-Y measured by the BGYRO-X response to a BET-Y pulse than any of the three individual controllers. 

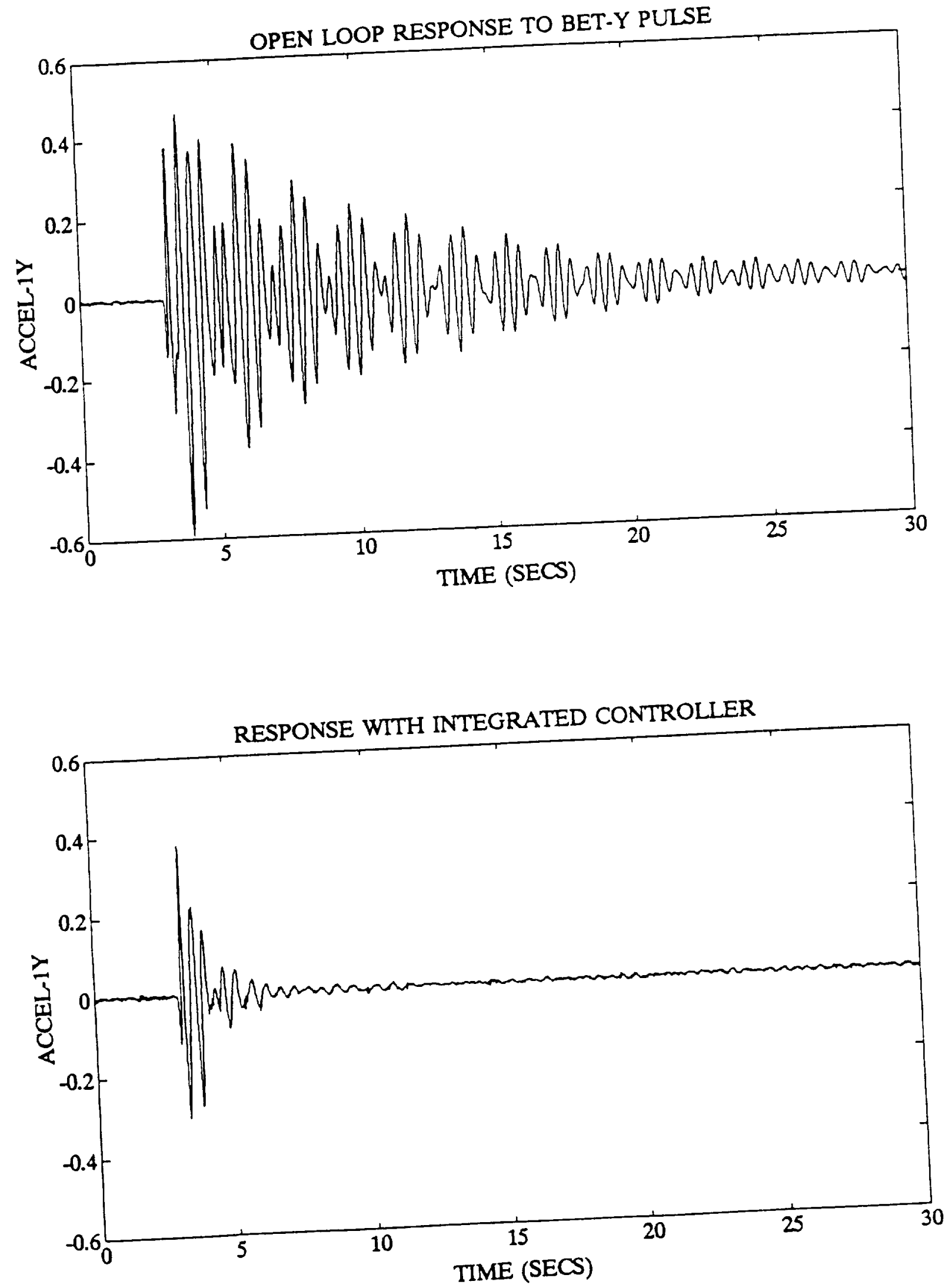
Figure 5.1.9. The integrated controller provided greater improvement in the beam damping as
measured by the ACCEL-1Y reponse to a BET-Y pulse than any of the three individual controllers. 

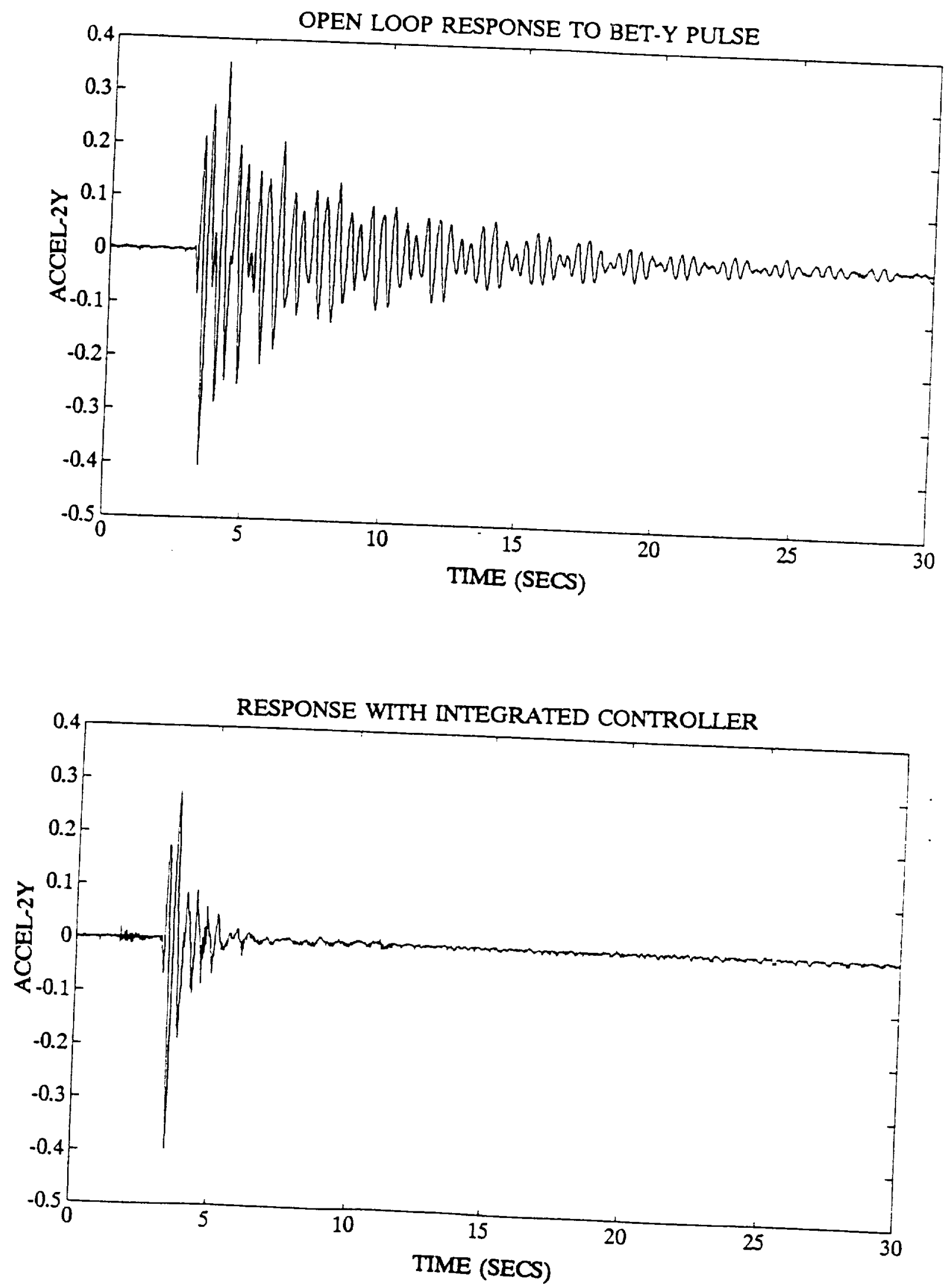

Figure 5.1.10. The integrated controller provided greater improvement in the beam damping controllers. 


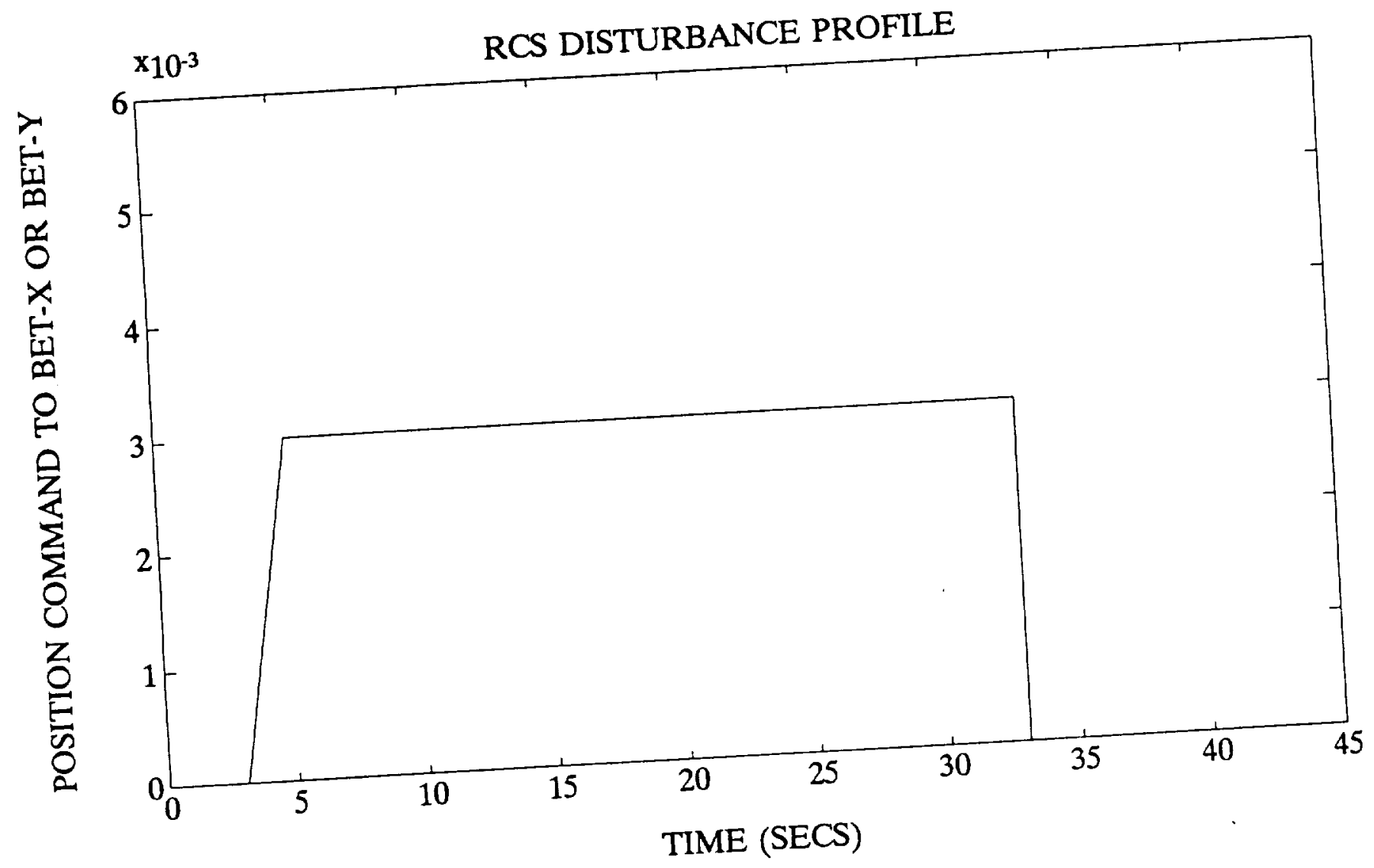

Figure 5.2.1. The RCS disturbance profile begins with a ramp and ends with a downward step. 

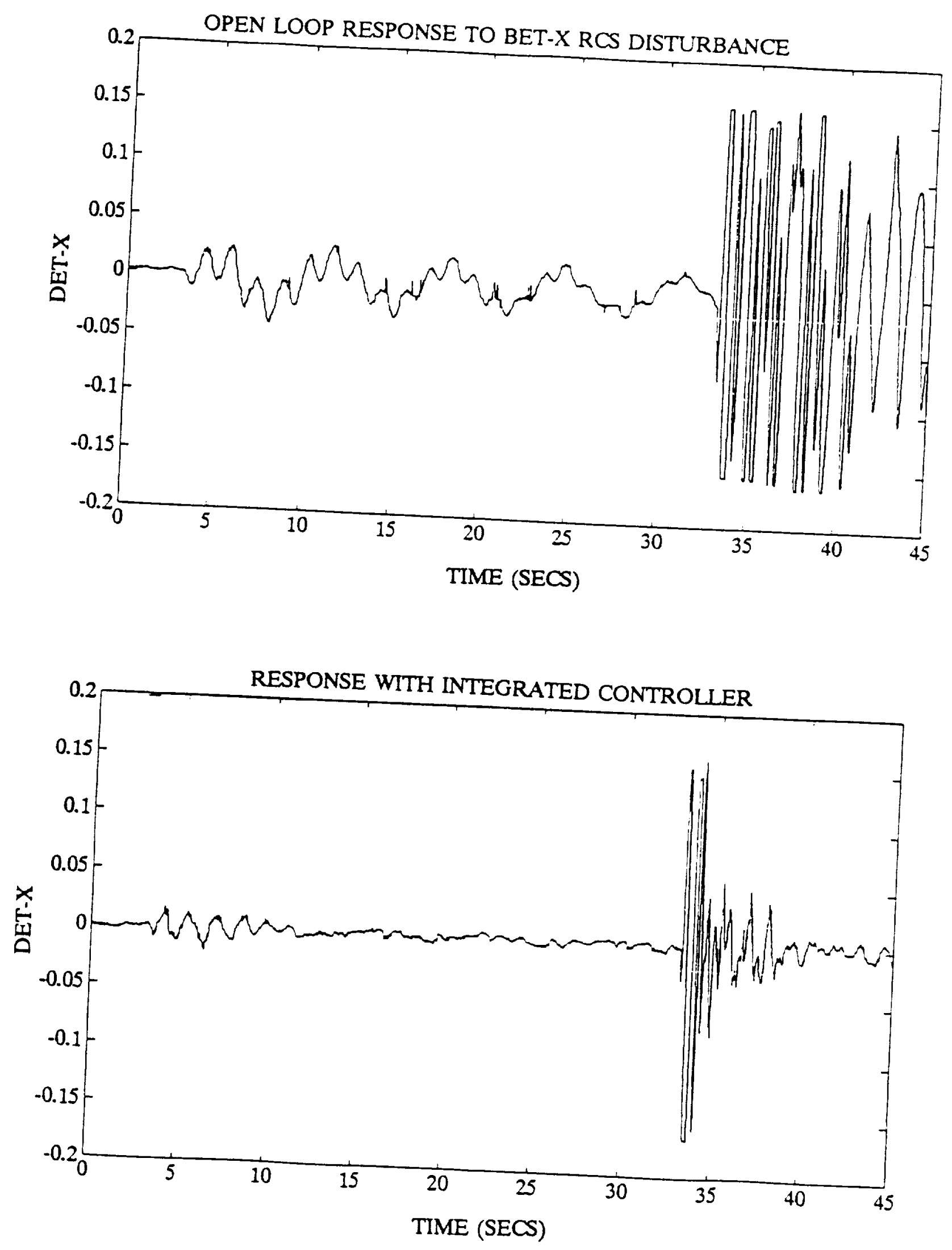

Figure 5.2.2. For a BET-X RCS disturbance the integrated controller provided substantial im-
provement in the DET-X response. 

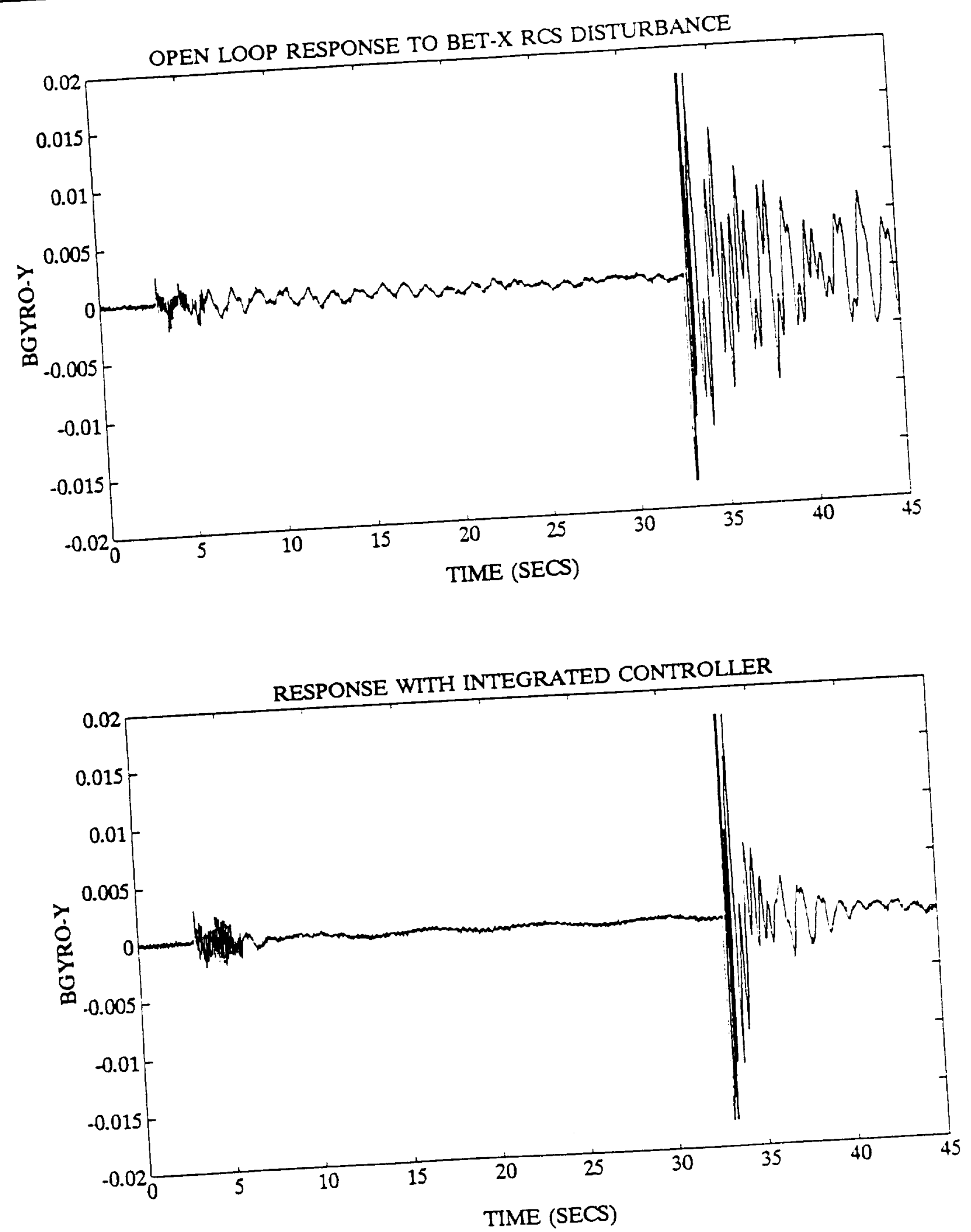

Figure 5.2.3. For a BET-X RCS disturbance the integrated controller provided substantial improvement in the BGYRO-Y response. 


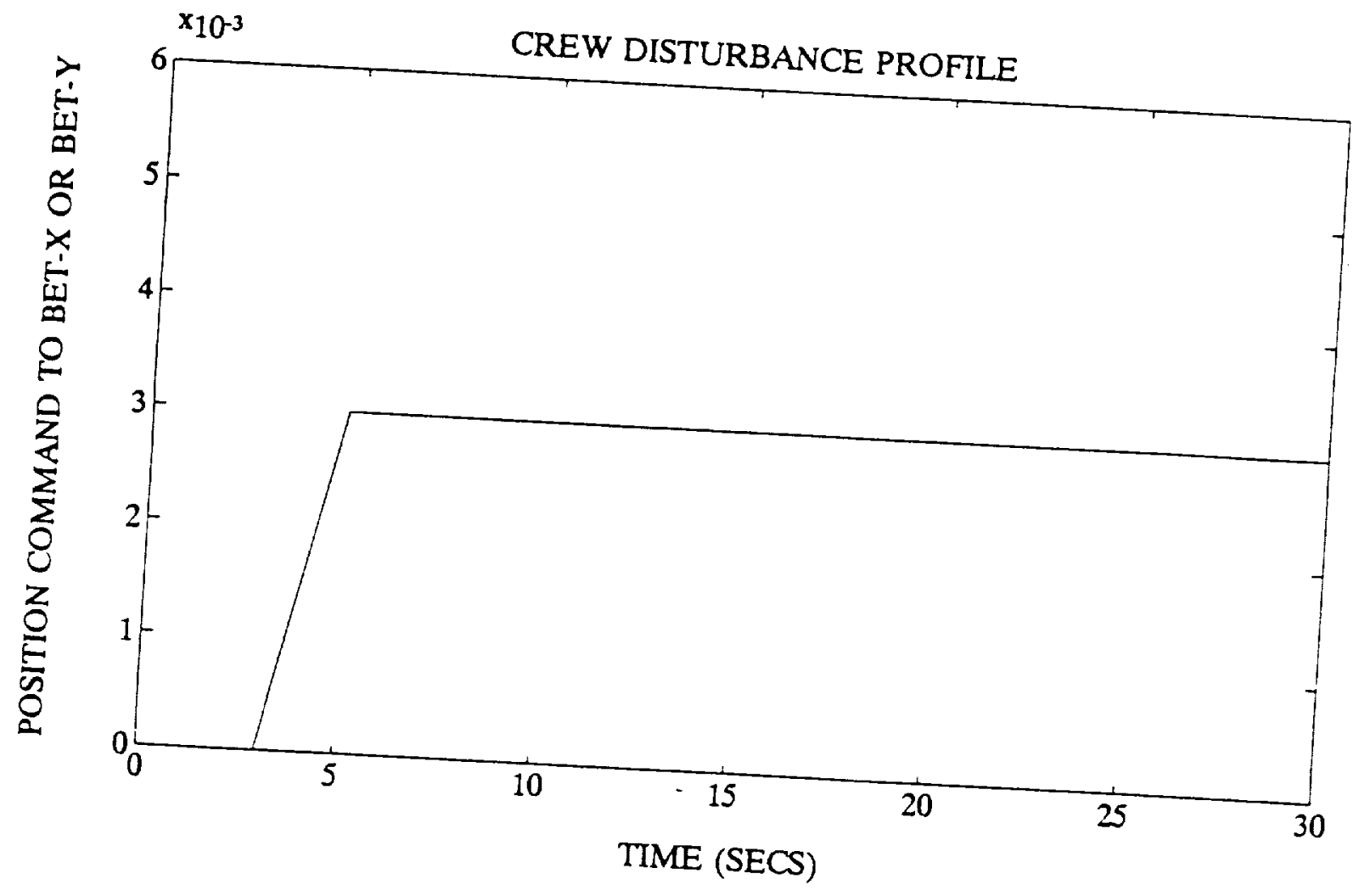

Figure 5.3.1. The Crew disturbance profile begins with a ramp and is constant after 5 seconds. 

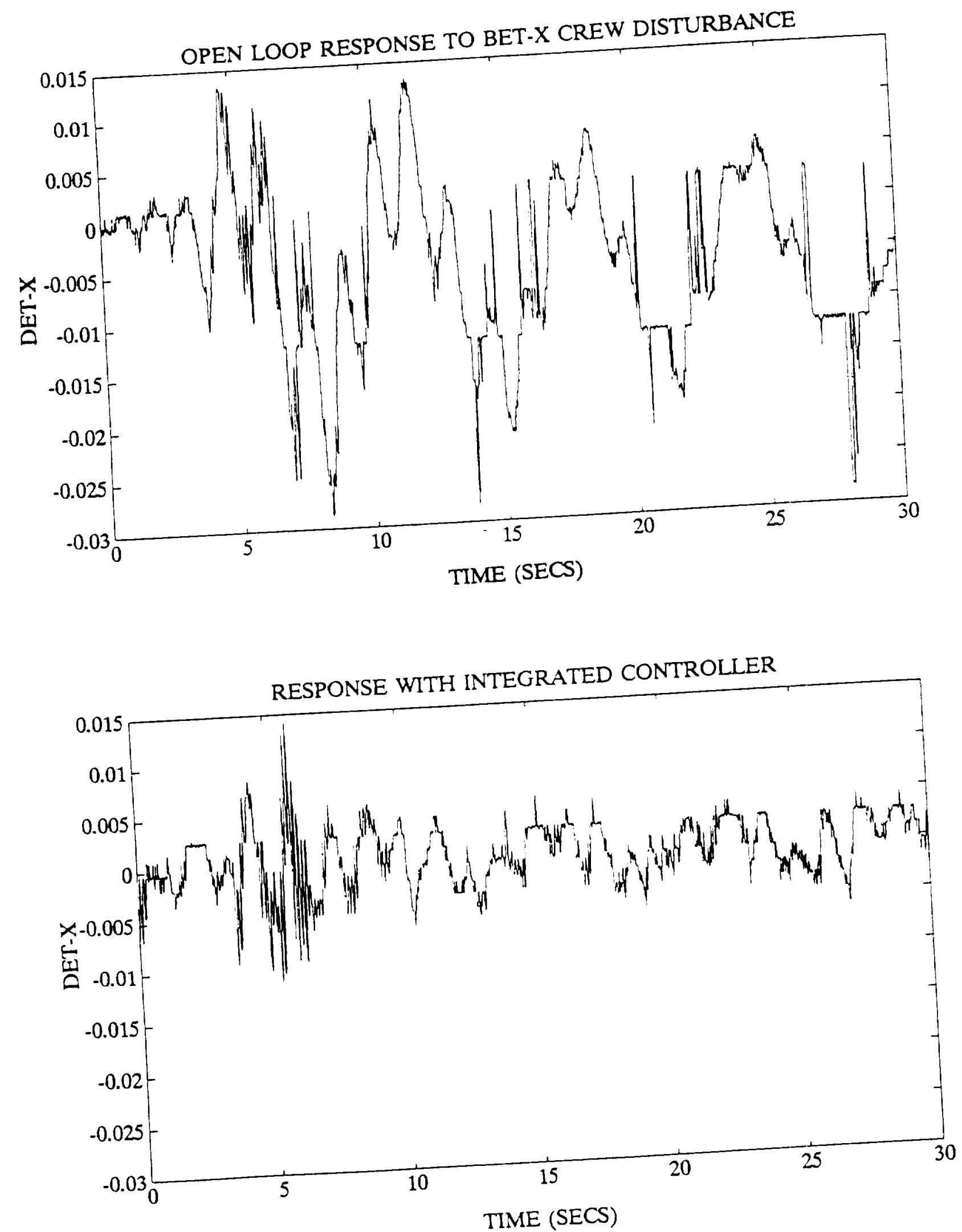

Figure 5.3.2. For a BET-X CREW disturbance the integrated controller provided substantial improvement in the DET-X response. 


\section{CLOSING REMARKS AND CONCLUSIONS}

This experiment has demonstrated successful control system design and implementation for the This experiment has demonstrated successful control system
ACES testbed, a flexible structure dynamically traceable to future space missions. This experiment
has also provided validation of the Maximum Entropy/Optimal Projection approach to control design.

In this project a major challenge was developing models with sufficient fidelity to support high performance control system design. This challenge will of course be found in most projects involving control design for flexible structures. It is important that the control designer not accept models without careful evaluation. It is the designer's responsibility to acquire modeling information that is sufficient to allow achievement of the control design objectives. This project also stressed the importance of modeling the actuator and sensor dynamics as well as the computational delay. For the ACES structure these aspects caused substantial phase delay and, in general, must be carefully considered in the development of control design models.

The results of this experiment also illustrate that simple controllers (i.e., reduced order and decentralized controllers) can provide very significant performance improvement for some flexible structure control problems. The total design model contained 45 states. However, the integrated controller contained only 28 states and had a decentralized architecture. This reduction in controller complexity is very important for the development of practical controllers due to substantial limits on throughput capability of even the most advanced spaced-qualified processors.

Another very helpful benefit of reduced complexity controllers is that they are easier to analyze which can save significant cost and time in both design and implementation.

In conclusion, we believe that a practical control design and implementation approach is to start with "simple" controllers and increase controller complexity as needed to increase performance.

Acknowledgements. We thank John Sharkey and Alan Patterson of NASA MSFC and Victoria Jones and Micheal Dendy of Control Dynamics Corporation. Without their excellent cooperation and support this project could not have been successful. We are also very grateful to Linda Smith for doing an excellent job in helping us to prepare this manuscript and Allen Daubendiek for helping us to develop the ERA models. 


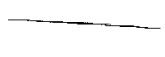




\section{References}

1. D. S. Bernstein and D. C. Hyland, "Optimal Projection for Uncertain System (OPUS): A Unified Theory of Reduced-Order, Robust Control Design," in Large Space Structures: Dynamics and Control, S. N. Atluri and A. K. Amos, eds., Springer-Verlag, New York, 1988.

2. D. C. Hyland, "Minimum Information Stochastic Modelling of Linear Systems with a Class of Parameter Uncertainties," Proc. Amer. Contr. Conf., pp. 620-627, Arlington, VA, June 1982. Structural Systems," Proc. Amer. Contr. Conf., pp. 680-688, Arlington, VA, June 1982

4. D. C. Hyland and D. S. Bernstein, "The Optimal Projection Equations for Fixed-Order Dynamic Compensation," IEEE Trans. Autom. Contr., Vol. AC-29, pp. 1034-1037, 1984. namic Compensation," IEEE Trans. Automion to
5. D. S. Bernstein and D. C. Hyland, "The Optimal Projection/Maximum Entropy Approach to
Designing Low-Order, Robust Controllers for Flexible Structures," Proc. IEEE Conf. Dec. Contr., pp. 745-752, Fort Lauderdale, FL. December 1985.

6. D. S. Bernstein and S. W. Greeley, "Robust Controller Synthesis Using the Maximum Entropy Design Equations," IEEE Trans. Autom. Contr., Vol. AC-31, pp. 362-364, 1986.

7. D. S. Bernstein, L. D. Davis and S. W. Greeley, "The Optimal Projection Equations for FixedOrder, Sampled-Data Dynamic Compensation with Computation Delay," IEEE Trans. Autom. Contr., Vol. AC-31, pp. 859-862, 1986. Contr., Vol. AC-31, pp. 859-862,
8. D. S. Bernstein, L. D. Davis, and D. C. Hyland, "The Optimal Projection Equations for
Reduced-Order, Discrete-Time Modelling, Estimation and Control," AIAA J. Guid. Contr. Dyn., Vol. 9, pp. 288-293, 1986.

9. S. Richter and E. G. Collins, Jr., "A Homotopy Algorithm for Reduced Order Compensator Design Using the Optimal Projection Equations," Proc. 28th IEEE Conf. Decis. Contr., pp. 506-511, Tampa, FL, Dec. 1989. 506-511, Tampa, FL, Dec. 1989.
10. D. S. Bernstein and D. C. Hyland, "The Optimal Projection Approach to Robust, Fixed-
Structure Control Design," Mechanics and Control of Space Structures, J. L. Junkins, Ed., AIAA Publication, 1990, to appear.

11. R. D. Irwin, V. L. Jones, S. A. Rice, S. M. Seltzer and D. J. Tollison, Active Control Tech- 
nique Evaluation for Spacecraft (ACES), Final Report to Flight Dynamics Lab of AF Wright
Aeronautical Labs, Report No. AFWAL-TR-88-3038, June 1988.

12. J. N. Juang and R. S. Pappa, "An Eigensystem Realization 1988

Identification and Model Reduction," J. Guid. Contr. Dyn., Vol 8 , Pp. $620-627,1985$.

13. J. N. Juang and R. S. Pappa, "Effects of Noisen

system Realization Algorithm," J. Guid. Contr. Dyn. Vodal Parameters Identified by the Eigen. Guid. Contr. Dyn., Vol. 9, pp. 294-303, 1986. 
Appendix A Control Gains for the Eight System Loops.

This Appendix presents the gains of the control laws described in Section 4. It is assumed that each control law is of the form

$$
\begin{aligned}
x_{c}(k+1) & =A_{c} x_{c}(k)+B_{c} y(k) \\
u(k) & =C_{c} x_{c}(k)+D_{c} y(k)
\end{aligned}
$$

Control Gains for the IMC-Y to DET-X Loop

$$
C_{c}=\left[\begin{array}{ll}
-2.00190 .90601 .0000
\end{array}\right], \quad D_{c}=1
$$

Control Gains for the IMC-X to DET-Y Loop

$$
\begin{aligned}
A_{c} & =\left[\begin{array}{ccc}
-0.1179 & -0.0168 & 0 \\
1.0000 & 0 & 0 \\
-2.0202 & 0.9246 & 1.0000
\end{array}\right], \quad B_{c}=\left[\begin{array}{l}
1 \\
0 \\
1
\end{array}\right] \\
C_{c} & =\left[\begin{array}{lll}
-2.0202 & 0.9246 & 1.0000
\end{array}, \quad D_{c}=1\right.
\end{aligned}
$$

Control Gains for the AGS-Y to BGYRO-Y Loop

$$
\begin{aligned}
& \text { Control Gains for the AGS-Y to BG } \\
& A_{c}=\left[\begin{array}{cccccc}
0.1781 & 0.8596 & 0 & 0 & 0 & 0 \\
-0.8596 & 0.3728 & 0 & 0 & 0 & 0 \\
-0.1219 & -0.0889 & -0.8281 & 0.5279 & 0 & 0 \\
0.3744 & 0.2731 & -0.5279 & -0.6211 & 0 & 0 \\
-0.1802 & -0.1315 & -0.0293 & -0.0901 & 0.3293 & 0 \\
-0.1252 & -0.0914 & -0.0204 & -0.0626 & 0.9236 & -0.9800
\end{array}\right], \quad B_{c}=\left[\begin{array}{c}
0.2754 \\
-0.2010 \\
0.2642 \\
-0.8113 \\
0.3906 \\
0.2714
\end{array}\right] \\
& C_{c}=\left[\begin{array}{lllll}
175.3127 .9 & 28.587 .6 & 1293.0-1372.0
\end{array}\right], D_{c}=8.8900
\end{aligned}
$$

Control Gains for the AGS-X to BGYRO-X Loop

$$
\begin{aligned}
& \text { Gains for the AGS-X to BGYRO-X Loop } \\
& A_{c}=\left[\begin{array}{cccc}
0.1125 & 0.6785 & 0 & 0 \\
-0.6785 & 0.5862 & 0 & 0 \\
-0.1779 & -0.1085 & -0.1599 & 0.8401 \\
0.1779 & 0.1085 & -0.8401 & -0.1599
\end{array}\right], \quad B_{c}=\left[\begin{array}{c}
0.6699 \\
-0.4086 \\
0.3451 \\
-0.3451
\end{array}\right] \\
& C_{c}=\left[\begin{array}{llll}
-198.2681 & -120.9193 & -178.2161 & -178.2161
\end{array}\right], \quad D_{c}=384.6600 \\
& \text { Gains for Each Colocated LMED Loop }
\end{aligned}
$$

Control Gains for Each Colocated LMED Loop

$$
\begin{aligned}
& A_{c}=\left[\begin{array}{ccc}
0.9987 & 0 & 0 \\
0.0117 & 0.9875 & 0 \\
0.0004 & 0.0606 & 0.9392
\end{array}\right], \quad B_{c}=\left[\begin{array}{l}
1.0000 \\
0.0177 \\
0.0005
\end{array}\right] \\
& C_{c}=\left[\begin{array}{ll}
0.0002 & 0.0341-0.0343
\end{array}\right], \quad D_{c}=3.0343 e-04
\end{aligned}
$$


